\title{
LEO Satellites and Frequency Synchronization
}

\author{
by \\ Yuntian Luo \\ A thesis submitted to the Faculty of Graduate and Postdoctoral Affairs in partial \\ fulfillment of the requirements for the degree of \\ Master of Applied Science in Electrical and Computer Engineering \\ Department of Electronics \\ Carleton University \\ Ottawa, Ontario \\ September 2020 \\ Copyright @2020 \\ Yuntian Luo
}




\section{Abstract}

Using the parameters provided by the project Telesat LEO, this thesis addresses the Doppler frequency shift encountered when an earth terminal station receives signals from a LEO satellite. To achieve frequency synchronization, there is a necessity to construct a Costas loop to demodulate the data in the receiver. However, since the frequency shift produced by the Doppler phenomenon is very large, the challenge becomes achieving and speeding up the acquisition time of the Costas loop.

Based on the theoretical foundations of the PLL, this thesis reviews the theory of BPSK and QPSK Costas loops. Then the thesis creates a new way of achieving fast synchronization for a BPSK Costas loop by adding a quadri-correlator in one of the Costas loop arms. To further optimize the synchronization, the combination of a traditional BPSK Costas loop and a BPSK Costas loop with a quadri-correlator is then introduced, which guarantees both stability and rapidity of acquisition. The construction of a QPSK Costas loop is significantly different from that of a BPSK Costas loop. The thesis then shows that the addition of a quadri-correlator cannot be used in a QPSK Costas loop, but that the synchronization time can still be reduced by changing the parameters in the loop filter. 


\section{Acknowledgement}

Firstly, I want to extend my great appreciation to my supervisor, Professor Jim Wight. When I received the admission to Carleton University, I was a course-based graduate. After taking the course Phase-locked Loop taught by Professor Wight, I become interested in this area, and admired his knowledge and kindness. This motived me to think about transferring from M.Eng to M.ASc. After discussing with Professor Wight and getting his admission, I successfully changed to be a thesis student to research in the area I like. He is always there to encourage me and clear away my confusion even during the pandemics. I really hope he and his family will be in happiness all life long.

Secondly, I would like to thank my parents. Thank you for supporting, caring for and encouraging me without any doubt. Especially for my father, he always talks with me about the philosophy of life to release the pressure and anxiety that I have and try to give me confidence in my academics.

Finally, I want to thank all my relatives, friends, and the staff at Carleton University who have educated or helped me. Due to the appearance of Covid-19, the year 2020 became unique, which brought trauma to this world. I hope all people can value their current life and believe that if citizens all the world can unite, we will beat the virus and get back to a better life. 


\section{Table of Contents}

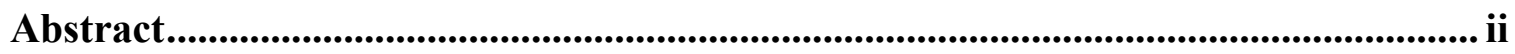

Acknowledgement ........................................................................................................ii

Table of Contents .............................................................................................................. iv

List of Figures................................................................................................................................. vi

Nomenclature ........................................................................................................................ ix

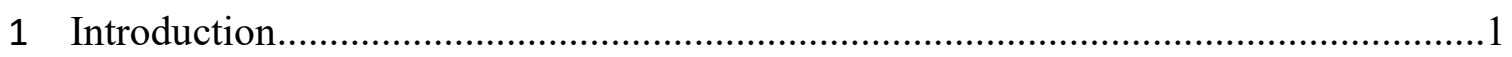

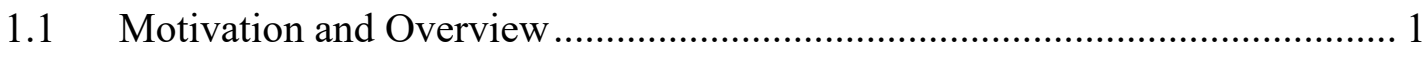

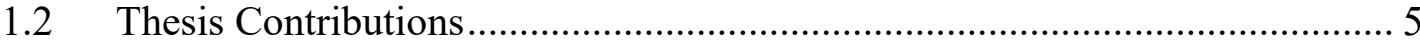

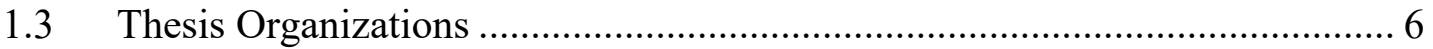

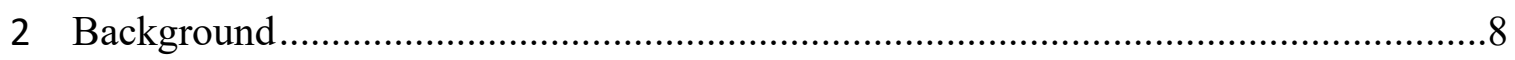

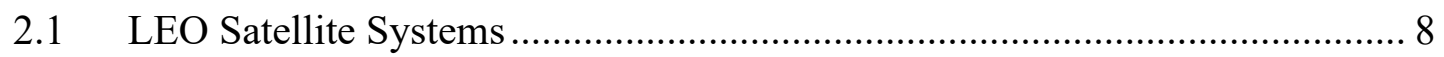

2.2 Doppler Frequency Shift in Satellite Communication ................................... 10

2.3 Doppler Frequency Shift for a LEO ........................................................ 17

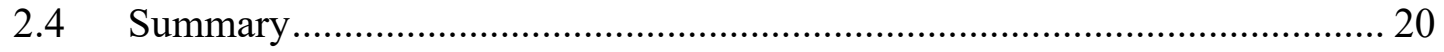

3 BPSK Costas Loop and Natural Acquisition............................................................22

3.1 Natural acquisition for BPSK Costas loop with 1st order filter ..................... 24

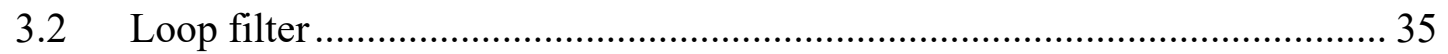

3.3 BPSK Costas loop natural acquisition with second-order loop filter.............. 41

3.4 Fast Methods for Achieving Acquisition....................................................... 46

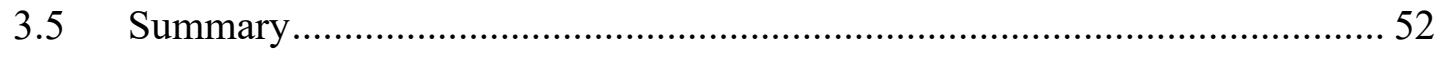

4 QPSK Costas Loop and Natural Acquisition..........................................................54 
4.1 Basic theory for QPSK Costas loop .......................................................... 54

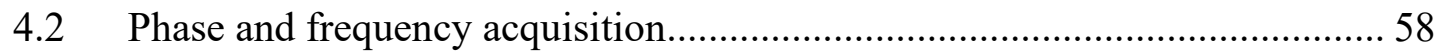

4.3 Fast Acquisition and the Impossibility of using a quadri-correlator in QPSK66

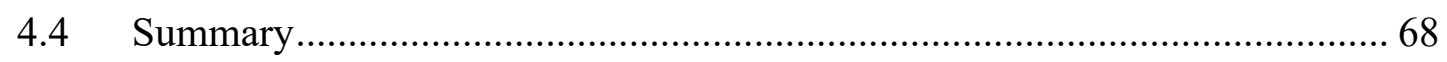

5 Doppler Frequency Tracking for a LEO ..............................................................

5.1 Doppler frequency shift tracking for BPSK signals ...................................... 70

5.2 Doppler frequency shift tracking for QPSK signals ....................................... 73

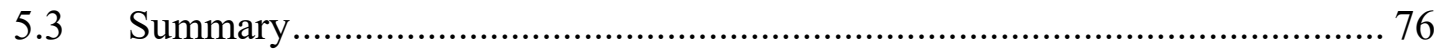

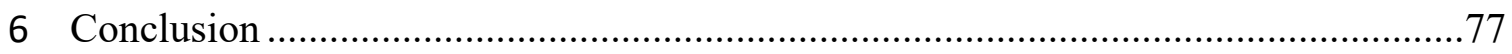

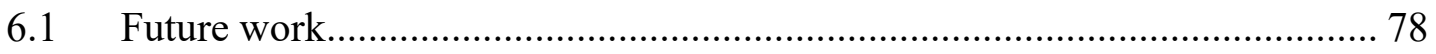

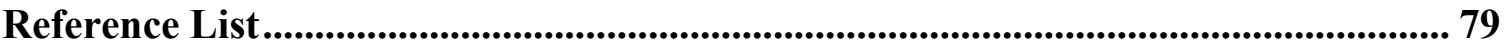




\section{List of Figures}

Figure 1-1 Basics of PLL 3

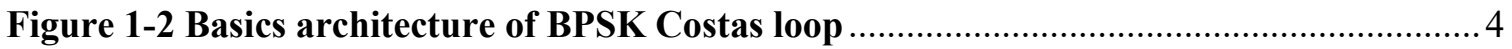

Figure 2-1 The characters of Telesat LEO constellation [10] .............................................

Figure 2-2 The distribution diagram of Telesat LEO global constellation for polar orbit and inclined orbit [10]

Figure 2-3 The communicating geometry between the terminal station and a LEO satellite

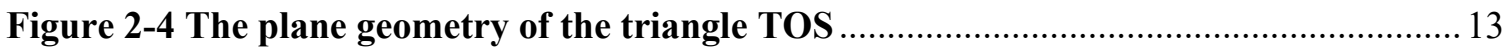

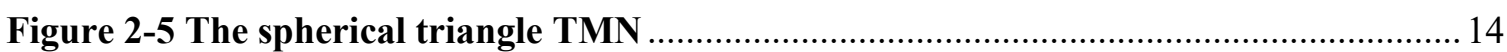

Figure 2-6 Visibility window, the relationship between duration time (t) and the maximum elevation angle (degree) for a LEO satellite in inclined orbit

Figure 2-7 The higher resolution duration time for a LEO satellite when the maximum elevation angle is 30 degree

Figure 2-8 The normalized Doppler frequency shift for a LEO satellite in inclined orbit

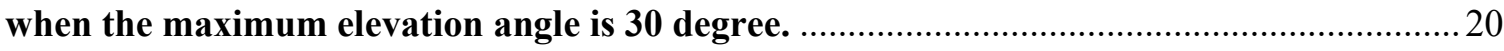

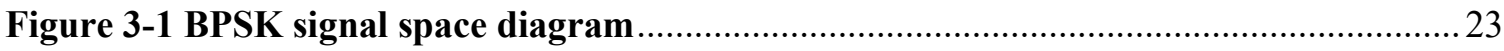

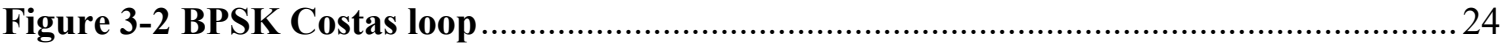

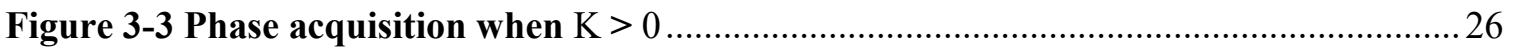

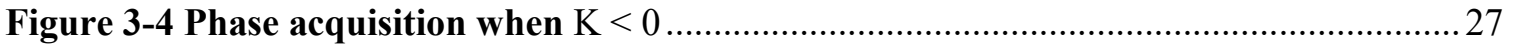

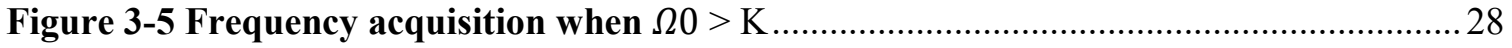

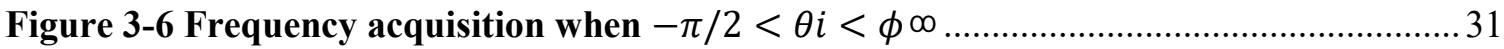

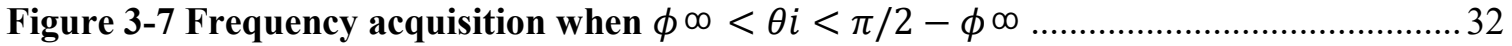

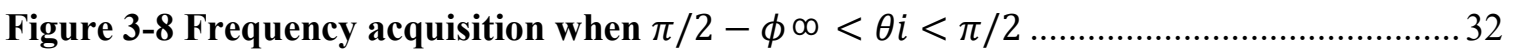

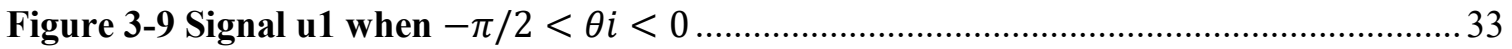

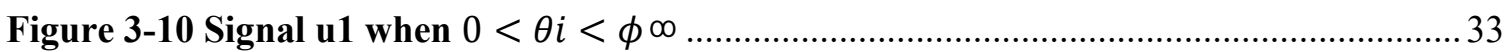

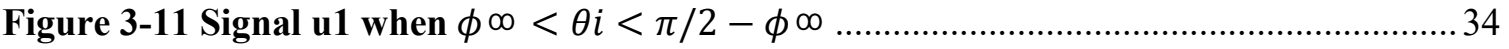

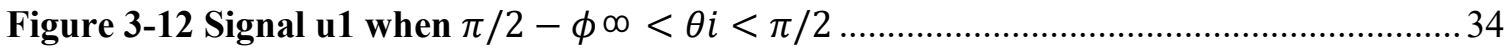


Figure 3-13 The circuit structure of integrator with phase-lead correction..... 35

Figure 3-14 Bode plot for integrator with phase-lead correction 36

Figure 3-15 The phase degree for Bode plot for integrator with phase lead correction when magnitude is zero 37

Figure 3-16 Comparison between the two Bode plots with two different values of damping constant $\zeta$ .38

Figure 3-17 Frequency step responses for different values of damping constant $\zeta \ldots \ldots \ldots \ldots \ldots .41$

Figure 3-18 Costas loop circuit design in MATLAB Simulink 42

Figure 3-19 The simulation of BPSK data in Simulink (the horizontal axis denotes time with the unit of second, the vertical axis denotes the amplitude, which are applied for all the figures in the Simulink result) 42

Figure 3-20 Turning point of Bode plot for integrator with phase-lead correction 44 Figure 3-21 Simulation of input before VCO in Simulink .45 Figure 3-22 Comparison between BPSK and demodulated BPSK signals with Costas loop 46 Figure 3-23 Comparison between BPSK and demodulated BPSK by using Costas loop with a smaller value of $\tau 1$ 47

Figure 3-24 The Costas loop with adding a quadri-correlator in I arm 48 Figure 3-25 A Costas loop with a quadri-correlator circuit design in MATLAB Simulink . 50 Figure 3-26 Comparison between BPSK and demodulated BPSK by using a Costas loop with a quadri-correlator .50

Figure 3-27 The circuit construction of combing a Costas loop and a Costas loop with a quadri-correlator in Simulink

Figure 3-28 Comparison between BPSK and BPSK demodulated by the optimized a Costas loop with a quadri-correlator .52

Figure 4-1 Signal space diagram for QPSK. .54

Figure 4-2 The diagram of a QPSK Costas loop .55

Figure 4-3 The piecewise function of output of the phase detector for a QPSK Costas loop 58 Figure 4-4 Simulink for a QPSK Costas loop in MATLAB .59

Figure 4-5 Data for $m(t)$ and $n(t)$... .59

Figure 4-6 The input of the QPSK. 60

Figure 4-7 The simulation of demodulated data for QPSK Costas loop phase acquisition .. 62 Figure 4-8 The test for the multiplication between sign function and sinusoidal function signals. 
Figure 4-9 The Simulation results for testing the frequency change of different process in QPSK Costas loop

Figure 4-10 The demodulated data for frequency acquisition for a QPSK Costas loop .......65

Figure 4-11 The waveform before the loop filter and the VCO ......................................... 65

Figure 4-12 The Demodulated QPSK signals with smaller value of $\tau 1$.............................66

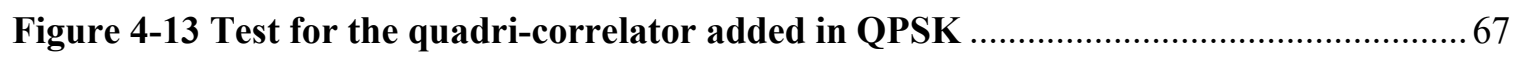

Figure 4-14 The simulation results for testing the QPSK signals with quadri-correlator ....68

Figure 5-1 Doppler sift tracking with BPSK Costas loop in Simulink .................................. 72

Figure 5-2 The simulation for demodulation of BPSK data when the terminal station

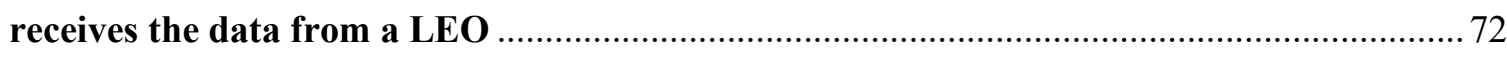

Figure 5-3 The simulation of the signal after the three filters. (1) the signal after the loop filter of the quadri-correlator Costas loop (2) the signal after the loop filter of the traditional Costas loop (3) the signal of the combination of two loop filters (the numbers are

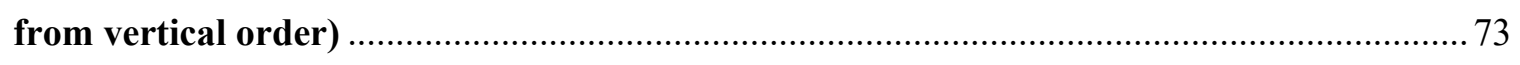

Figure 5-4 Doppler shift tracking with QPSK Costas loop in Simulink ............................... 74

Figure 5-5 The simulation for demodulation of QPSK data when the terminal station receives the data from Telesat LEO (1) The demodulated signals of $m(t)$ (2) The

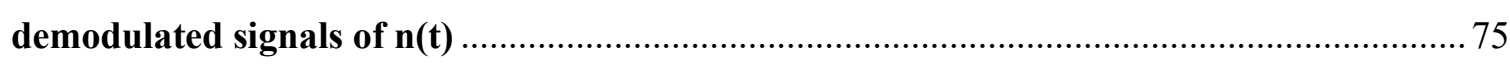

Figure 5-6 The simulation of the signals before and after the loop filter in QPSK Costas loop. (1) the signals after before the loop filter (2) the signals after the loop ....................... 75 


\section{Nomenclature}

$\begin{array}{ll}\text { 5G } & \text { Fifth generation technology standard for cellular networks } \\ \text { BPSK } & \text { Binary phase-shift keying } \\ \text { ECF } & \text { Earth center fixed } \\ \text { ECI } & \text { Earth centered inertial } \\ \text { GEO } & \text { Geostationary earth orbit } \\ \text { LEO } & \text { Low earth orbit } \\ \text { LO } & \text { Local oscillator } \\ \text { LPF } & \text { Low pass filter } \\ \text { PLL } & \text { Phase-locked loop } \\ \text { QPSK } & \text { Quaternary phase-shift keying } \\ \text { VCO } & \text { Voltage-controlled oscillator }\end{array}$




\section{Chapter 1}

\section{Introduction}

\subsection{Motivation and Overview}

\section{Low Earth Orbit (LEO) satellite}

As the development and demand for satellite traffic has increased, Low Earth Orbit (LEO) satellites have gain popularity in recent years. The LEO satellites have mostly been used in scientific applications, including analyses of climate change, remote sensing of ocean and navigation system [1]. Being an earth-centered orbit, a LEO's altitude is less than 2,000 $\mathrm{km}(1,200 \mathrm{mi})$, which is different from Geostationary Earth Orbit (GEO) satellites with an altitude of $35,786 \mathrm{~km}$, located over the equator, circulating synchronously with the earth [2]. Satellites have been used for a long time; nevertheless, there are still new and revolutionary techniques in the communication area.

During the last few years in telecommunication, $5 \mathrm{G}$, known as fifth generation technology standard for cellular networks, began to rise. 5G aims to achieve communications with broadband high speed, ultra-reliable and low-latency services. 5G plays a key role to facilitate economic and social globalization. The high demand also brings more challenges that $5 \mathrm{G}$ needs to face especially for global and seamless connection. At this time, the integration of satellites and terrestrial network can be an efficient technique to achieve the globalization requirements. Since the satellites have a large footprint, they can enlarge the terrestrial networks in most of the places on the earth. 
Although GEO satellites are suitable for global large-capacity coverage, the disadvantages that GEO shows seriously challenge the principle of 5G [3].

Apart from the higher power consumption for the communication, one obvious drawback of GEO satellite communication system is its long propagation delay, which can reach approximately $120 \mathrm{~ms}$ for one-way transmission [4]. This is inconvenient for any real-time link between users and terminal stations [2]. The advent of LEO satellites can remedy this issue and help satisfy the $5 \mathrm{G}$ revolution, since the distance from the terminal station is conspicuous shorter than for GEO satellites, meaning there will be shorter roundtrip delays and lower power consumption [5].

Because the LEO satellites are in a fast constant motion related to the earth, megaconstellation of LEO satellites can provide continuous and global coverage as they move [6], which can offer wireless communication anywhere, including the polar area of the earth. However, the design and structure of LEO satellite constellation need to be studied rigorously, since the mobility of LEO satellites can also bring handover management and location management challenges in contrast to GEO system [2].

\section{Phase-Locked Loop (PLL)}

In LEO satellite communication, since the fast movement exists among satellites or between the satellite and the terminal station, there will be Doppler frequency shift appearing when signals are received. In other words, upon arrival, the received waveform will show an obvious frequency offset between the transceivers. If the receiver cannot quickly and accurately track the frequency shift, the huge distortion appearing in the signal will make it impossible to demodulate the data. Consequently, the need of fast acquisition 
is necessary to track the Doppler frequency shift in LEO satellite communication. Thanks to the creation of phase-locked loop (PLL), once we determine the carrier frequency shift, the proper design of this circuit can track the frequency of received signal [7], demodulate the data and finish the wireless transmission with less distortion.

The invention of PLL theory can date back in 1923 by Appleton and in 1932 by de Bellescize; however, the theoretical explanation was not well established until in the late 1970s. After the rapid technical growth in integrated circuits (ICs), PLL gained wide use in various modern techniques [8]. Now, the PLL techniques have become more mature, and are used in power supplies, motor control systems, communication systems and electronic applications [9].

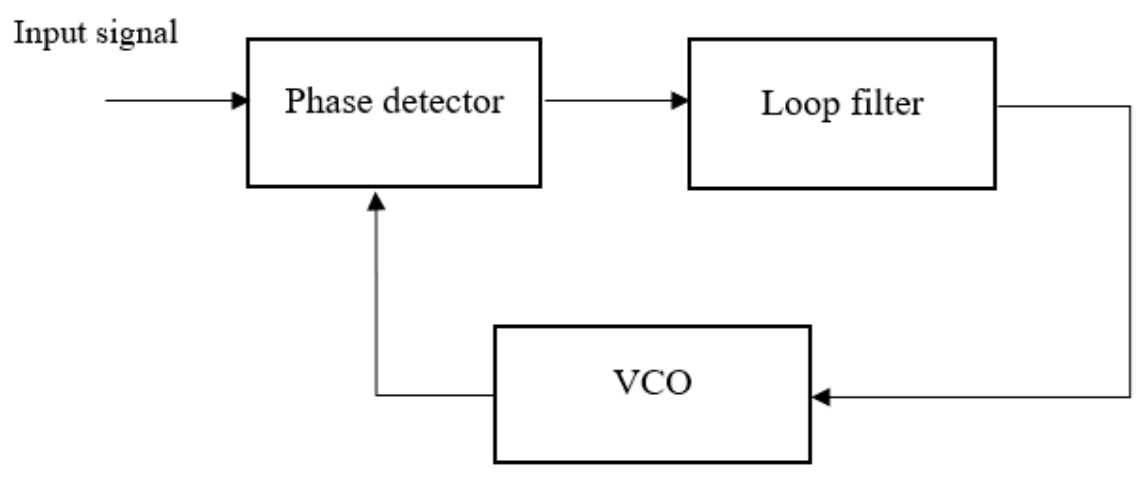

Figure 1-1 Basics of PLL

The use of PLLs in different areas means there are diverse designs of the PLL circuit, but the basics of PLL circuits remains the same. The PLL incorporates three elements, the phase detector, loop filter and voltage-controlled oscillator (VCO), as presented in Figure 1-1. 
After the input signal comes into the loop, the block of phase detector will compare the input signal and the signal produced by VCO. Then an error signal will pass trough the loop filter, producing a new signal which adjusts the VCO frequency to generate a signal that is close to the input. The cycle continues until the loop is locked. Although only three elements compose PLL, the details and categories in these three parts can be complicated and numerous. Especially for the phase detector, the block can contain various components.

One of the most important designs of PLL is the Costas loop and it has been widely used in synchronization in wireless communication. In LEO satellite communication, since the Doppler frequency shift brings the change of received frequency into the terminal station, the Costas loop is an optional choice for tracking the frequency and demodulating the data. The basics BPSK Costas loop architecture is shown in Figure 1-2:

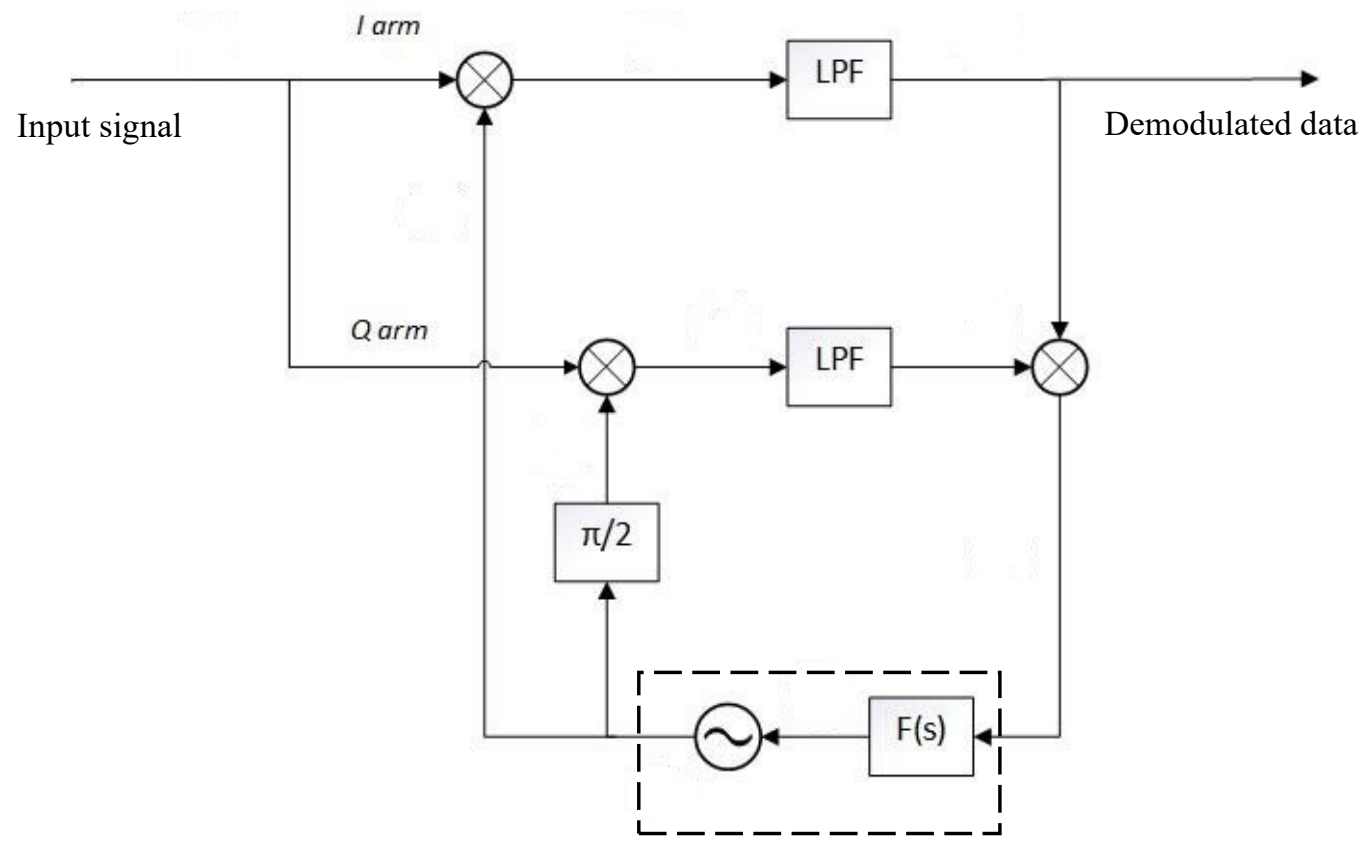

Figure 1-2 Basics architecture of BPSK Costas loop 
The elements in the dash line frame is the loop filter and VCO, and all the other blocks outside of dash line frame compose the phase detector. The input signal comes into the I and Q arm of the phase detector simultaneously. Upon mixing with the feedback from the VCO, and after passing the two identical low pass filters (LPFs), the signals are combined and passed through the loop filter to adjust the VCO like in a basic function PLL. For BPSK modulation, the data is taken from the I arm as shown in Figure 1-2.

Costas loops work well in tracking the changing frequency; nonetheless, the frequency offset is huge in satellite communication, which means an improvement for Costas loop becomes vital if we want to guarantee the accuracy and integrity of the transmitted information. In other words, auxiliary circuits to decrease the acquisition time for the Costas loop will be needed. In a Costas loop, creating a quadri-correlator in the I or Q arm can help accelerate frequency acquisition. Here we add a differential function in one of the arms, and the new circuit can adjust the VCO quickly. The details of the quadricorrelator will be discussed in Chapter 3 .

\subsection{Thesis Contributions}

This thesis contributes the ways of achieving rapid synchronization of data transmission between a terminal station and a LEO satellite. The thesis illustrates how the theory works for BPSK and QPSK Costas loops, and the parameters setting for the loops. In frequency synchronization, the thesis shows the way of creating a new Costas loop to achieve fast synchronization by adding a quadri-correlator. Also, a method of achieving both fast and stable synchronization by combing the traditional Costas loop and the new Costas loop with a quadri-correlator will be presented. The new designed BPSK Costas 
loop can finish synchronization at $0.1 \mathrm{~s}$ when the initial frequency difference is $1000 \mathrm{rad} / \mathrm{s}$. Finally, the new Costas loop can track the Doppler frequency shift occurring between a LEO satellite and the terminal station, demodulating the transmitted data successfully at $0.4 \mathrm{~s}$.

\subsection{Thesis Organizations}

The Chapter 2 Background first gives a brief introduction for a LEO satellite constellation and the new LEO satellite program, Telesat LEO. This section also mentions the planning parameters for the Telesat LEO, e.g. inclination, altitude, constellation categories and the use of spectrum. Then the time dependent Doppler frequency shift between a LEO satellite and a terminal station is derived in detail. Finally, how the exact Doppler frequency shift will change with time during the transmission between a LEO satellite and a terminal station is shown.

In Chapter 3, the thesis develops the natural acquisition for Costas loop. The first part gives the theoretical equations for the BPSK Costas loop with a $1^{\text {st }}$ order filter, including phase acquisition and frequency acquisition. On the basic of the theory of the $1^{\text {st }}$ order loop and the character of a $2^{\text {nd }}$ order filter, using an integrator with phase lead correction, the parameters of setting for $2^{\text {nd }}$ order Costas loop are presented. Using MATLAB Simulink, then the simulation of the BPSK Costas loop is shown. To improve the acquisition time, a new way of adding a quadri-correlator in a BPSK Costas loop is presented and the method of optimizing the quadri-correlator to guarantee both the speed and stability of Costas loop is shown finally. 
Similar to the process for the BPSK Costas loop, Chapter 4 presents the theory and the simulation of a QPSK Costas loop. However, due to the loop construction being different from BPSK Costas loop, the chapter then shows adding a quadri-correlator can result in increased sensitivity. If the parameters of the loop are not correct, the QPSK Costas loop fails to achieve demodulation; nevertheless, the acquisition time can is reduced by correctly selecting the parameters of the loop.

The link of the Doppler frequency shift of a LEO satellite and the designed BPSK and QPSK Costas loop is presented in Chapter 5. In this chapter, the achievable acquisition can be clearly shown here by using MATLAB Simulink.

A summary for the thesis and future work can be done for further optimizing the Costas loop is shown in Chapter 6. 


\section{Chapter 2}

\section{Background}

\subsection{LEO Satellite Systems}

There are some existing LEO satellite networks, for instance, Iridium, Teledesic, Globalstar and Ellipso, and each system has its own basic architecture concepts. As a result, the designing parameters including the orbit parameters, the network connectivity, the routing model and the coverage model must be chosen individually, which influences the system's ability and the service quality for users [2]. Since there is a huge technical and scientific potential in LEO satellite communication systems, many satellite companies are also starting their engineering plans for LEO satellite constellations. One of the biggest satellite companies is Telesat, a Canadian satellite communications company which schedules their LEO network, Telesat LEO, to be operational in 2022 . To meet the future needs in communication area, the Telesat LEO adheres to the concept of achieving the highest quality of service including both low latency with sub $100 \mathrm{msec}$ delay, and high throughput with, Gbps links and Tbps sellable capacity. The network also considers low cost and flexible connectivity to accomplish transformational economics and wireless connection both in global land and ocean areas [10]. 


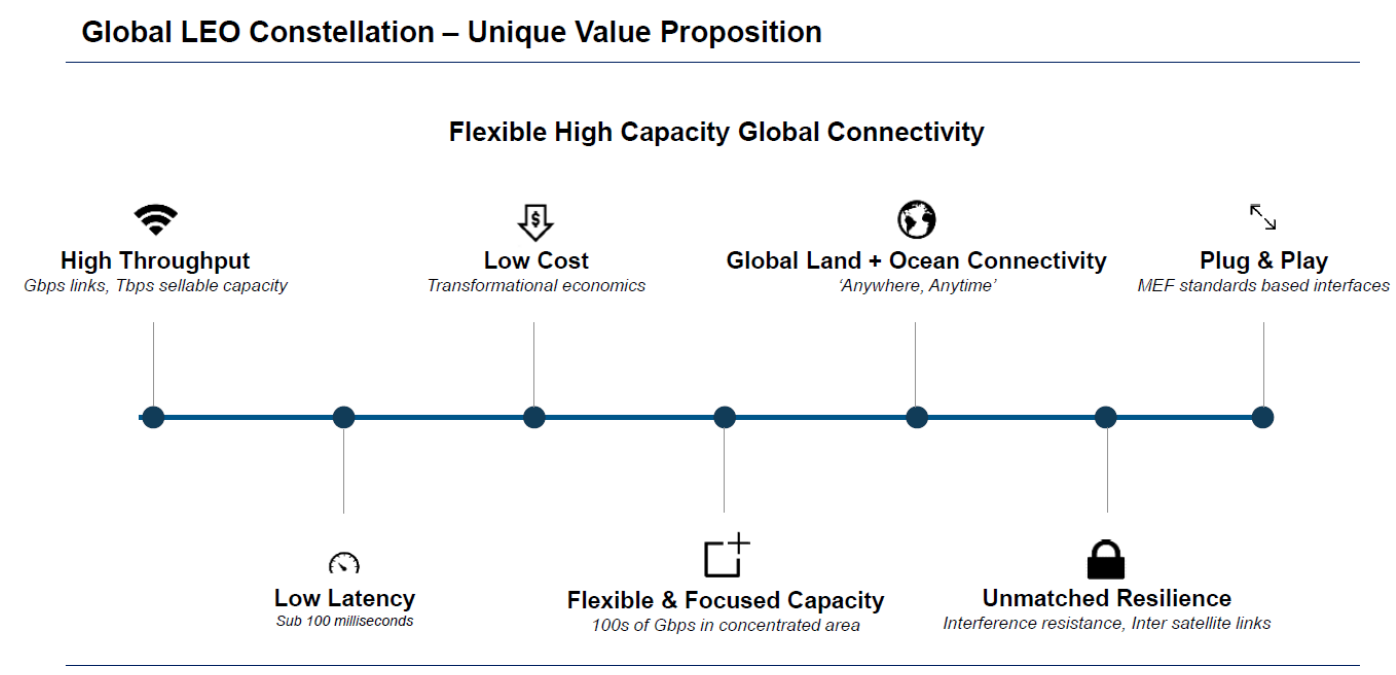

Figure 2-1 The characters of Telesat LEO constellation [10]

With the principle of global coverage and high demand capacity, there are two categories of Global LEO constellations in Telesat LEO, one of which is the Polar Orbit Constellation. In this plan, being in synchronous with the sun, 78 LEO satellites will be in the polar circular orbit with an altitude of $1000 \mathrm{~km}$, and an inclination angle of 99.5 degrees. There will be 6 orbital planes in total in the polar orbit, which are spaced 30 degrees about the equator. The second orbit category is the Inclined Orbit Constellation, and the augmented capacity constellation now has reached 440 satellites in Inclined Orbit with the altitude of $1,350 \mathrm{~km}$. There will be 20 planes in the inclined orbit with 50.0 -degree angle, with the planes spaced 9 degrees [10,11]. The design is shown in Figure 2-2.

Telesat LEO also shows advanced technology including using directing radiating antennas (DRA) with agile, hopping beams for flexible capacity and on-board processing (OBP) for efficient routing and around $4 \mathrm{GHz}$ of Ka-band spectrum. The optical intersatellite links (OISL) will also establish a global mesh network [10]. 


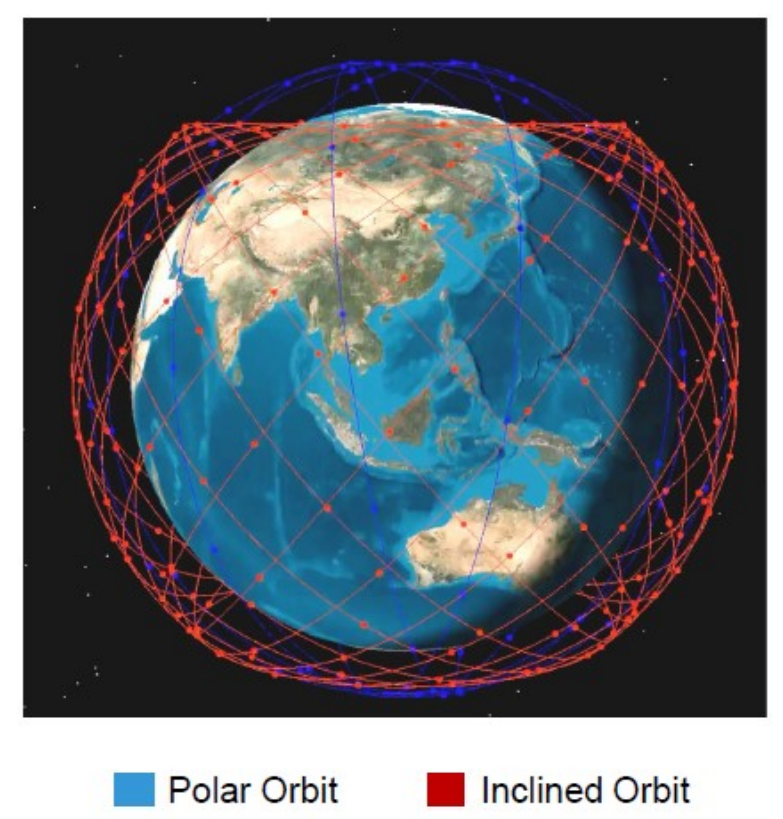

Figure 2-2 The distribution diagram of Telesat LEO global constellation for polar orbit and inclined orbit [10]

\subsection{Doppler Frequency Shift in Satellite Communication}

When an observer moves relative to a wave source, the frequency of the wave in relation to the observer changes, which is called a Doppler shift. Doppler frequency shift can be often seen in the communication system, and it is especially high if the receiver and the transmitter are not static and in obvious relative velocity [7]. In LEO satellite communication, since the velocity of satellites is high and non-geostationary, Doppler frequency shift becomes one of the most important parameters to consider. Consequently, the analysis of the Doppler frequency shift including estimation and compensation is significant [7,12].

In the satellite communication, the Doppler effect equation can be written as: 


$$
f=\frac{c}{c-\Delta v} f_{0}
$$

Where $\Delta v$ is velocity of the emitter with respect to the receiver, $f$ is the received frequency, $f_{0}$ is the emitted frequency, and $c$ is the speed of light. The equation can be further simplified as (2.2), if relative velocity $\Delta v$ is pretty small compared with c.

$$
f=\left(1+\frac{\Delta v}{c}\right) f_{0}
$$

Then the change of doppler frequency shift $\Delta f$ can be written as:

$$
\frac{\Delta f}{f_{0}}=\frac{\Delta v}{c}
$$

The above basic analysis of Doppler shift can be used as fundamental of the Doppler frequency shift in LEO satellite communication. Since the geometrics above the earth and the satellite pathway are complicated, the equation for the Doppler shift function needs to be derived carefully. One of the common ways of deriving the above Doppler shift equation is to observe the equation from a given terminal station and a maximum elevation angle. Also, the analysis strategy is to use earth center fixed (ECF) coordinate frame [12]. The figure of communication between terminal station and LEO satellite can be shown in Figure 2-3: 


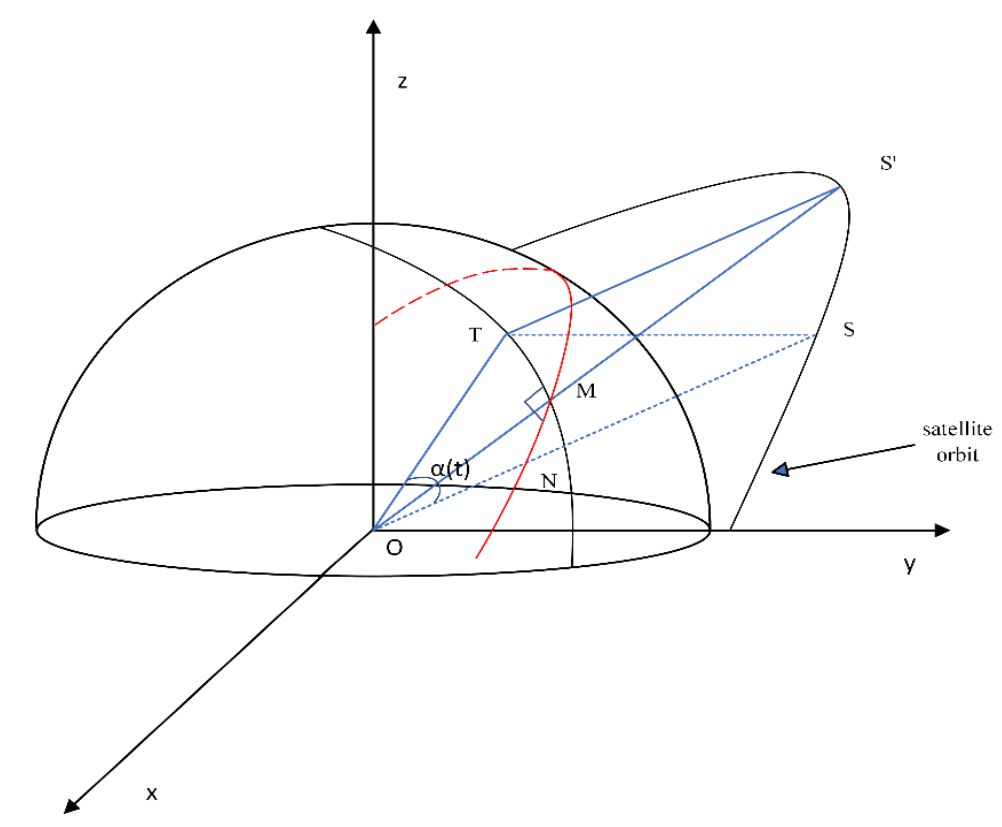

Figure 2-3 The communicating geometry between the terminal station and a LEO satellite

The figure shows the LEO satellite orbit compared with the terminal station, T. In Figure 2-3, $S$ and $S^{\prime}$ denote the position of the satellite at time $t$ and at the maximum elevation angle separately. $\mathrm{O}$ is the center of earth and the red orbit denotes the subsatellite. The points $\mathrm{M}$ and $\mathrm{N}$ are the intersection point between the subsatellite orbit and the line OS' or OS.

According to the equation (2.3), the normalized Doppler shift can be written as $\frac{\Delta v}{c}$, if the reference system is based on the static earth, $\Delta v$ will be the real velocity of the satellite in the direction of OS. Since the position of S is changing, the distance OS can be regarded as a function of time, $\mathrm{S}(\mathrm{t})$. Then the Doppler shift here can be written as [12]:

$$
\frac{\Delta f}{f_{0}}=-\frac{d S(t)}{c d t}
$$

To further derive the function $\mathrm{S}(\mathrm{t})$, it is necessary to make an analysis of triangle TOS, which is shown in Figure 2-4: 


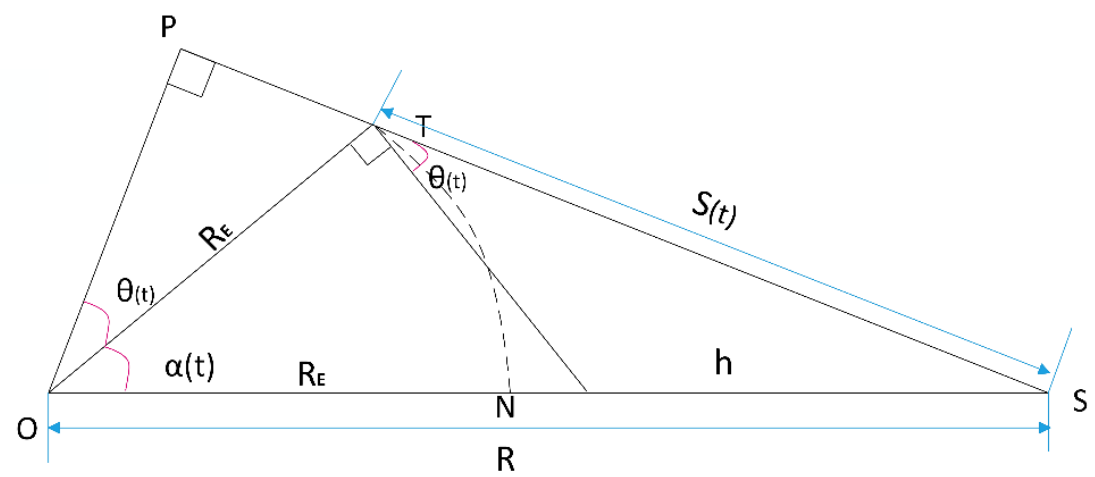

Figure 2-4 The plane geometry of the triangle TOS

Ignoring the small variations, in ECF frame, the satellite orbit can be regarded as a great-circle arc and the angular velocity of LEO satellite is constant during the visibility window. As a result, according to the cosine law of plane triangle, in triangle TOS, $\mathrm{S}(\mathrm{t})$ can be calculated by using $\alpha(t)$, the angle of $\angle N O T$, where $R_{E}$ represents the radius of the earth, $\mathrm{h}$ denotes the height of the satellite.

$$
s(t)=\sqrt{R_{E}^{2}+R^{2}-2 R_{E} R \cos \alpha(t)}
$$

Then differentiating both sides:

$$
\frac{d s(t)}{d t}=-\frac{R_{E} R}{\sqrt{R_{E}^{2}+R^{2}-2 R_{E} R \cos \alpha(t)}} \frac{d \cos \alpha(t)}{d t}
$$

In the Figure 2-4, $\theta(t)$ is the elevation angle from the aspect of terminal station at time, t. In the triangle $\triangle O P S$. The length of OP can be equal to $R \cos (\theta(t)+\alpha(t))$. The calculation of $\mathrm{OP}$ can also be represented in the triangle $\triangle O P T, \mathrm{OP}=R_{E} \cos (\theta(t))$. From the two equations we can have:

$$
R_{E} \cos (\theta(t))=R \cos (\theta(t)+\alpha(t))
$$

The triangle TMN in satellite plot is a spherical triangle, shown in Figure 2-5. 


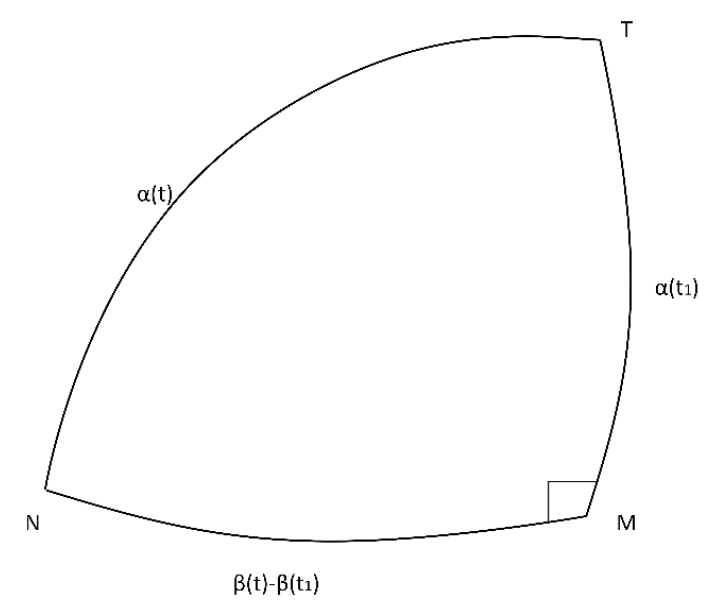

Figure 2-5 The spherical triangle TMN

In the Figure 2-5, the arc MN, NT and MT are all in big circles. $\mathrm{t}_{1}$ represents the time when the terminal station observes the satellite in the maximum elevation angle. $\beta(t)-\beta\left(t_{1}\right)$ is the angular distance of arc MN on the surface of the earth.

According to the spherical triangle law:

$$
\cos \alpha(t)=\cos \left(\beta(t)-\beta\left(t_{1}\right)\right) \cos \alpha\left(t_{1}\right)+\sin \left(\beta(t)-\beta\left(t_{1}\right)\right) \sin \alpha\left(t_{1}\right) \cos (\angle T M N)
$$

Since the point $\mathrm{M}$ is the point of intersection of the earth's surface and the connection between the earth's center and LEO satellite's position with the maximum elevation angle of the terminal station, meaning that $\angle T M N=90^{\circ}$. The equation can then be simplified into:

$$
\cos \alpha(t)=\cos \left(\beta(t)-\beta\left(t_{1}\right)\right) \cos \alpha\left(t_{1}\right)
$$

Differentiating equation 2.9, we can get the expression for the $\frac{d \cos \alpha(t)}{d t}$ :

$$
\frac{d \cos \alpha(t)}{d t}=-\sin \left(\beta(t)-\beta\left(t_{1}\right)\right) \cos \alpha\left(t_{1}\right) \frac{d \beta(t)}{d t}
$$


Combining equation 2.10 and equation 2.6, the expression of $\frac{d s(t)}{d t}$ can be written as a function of $\beta(t)$ :

$$
\frac{d s(t)}{d t}=\frac{R_{E} R \sin \left(\beta(t)-\beta\left(t_{1}\right)\right) \cos \alpha\left(t_{1}\right)}{\sqrt{R_{E}^{2}+R^{2}-2 R_{E} R \cos \left(\beta(t)-\beta\left(t_{1}\right)\right) \cos \alpha\left(t_{1}\right)}} \frac{d \beta(t)}{d t}
$$

Recall the equation 2.7 , and when the time $t$ is at the instant of maximum elevation, $\mathrm{t}$ becomes $\mathrm{t}_{1}$, then we can get:

$$
R_{E} \cos \left(\theta\left(t_{1}\right)\right)=R \cos \left(\theta\left(t_{1}\right)+\alpha\left(t_{1}\right)\right)
$$

The expression of $\alpha\left(t_{1}\right)$ can be derived from the equation:

$$
\alpha\left(t_{1}\right)=\cos ^{-1}\left(\frac{R_{E}}{R} \cos \theta\left(t_{1}\right)\right)-\theta\left(t_{1}\right)
$$

Combing the equations 2.11 and 2.13, the expression of $\frac{d s(t)}{d t}$ can be written as a function of time $t$ and maximum elevation angle $\theta\left(t_{1}\right)$.

$$
\frac{d s(t)}{d t}=\frac{R_{E} R \sin \left(\beta(t)-\beta\left(t_{1}\right)\right) \cos \left(\cos ^{-1}\left(\frac{R_{E}}{R} \cos \theta\left(t_{1}\right)\right)-\theta\left(t_{1}\right)\right)}{\sqrt{\left.R_{E}^{2}+R^{2}-2 R_{E} R \cos \left(\beta(t)-\beta\left(t_{1}\right)\right) \cos \left(\cos ^{-1}\left(\frac{R_{E}}{R} \cos \theta\left(t_{1}\right)\right)-\theta\left(t_{1}\right)\right)\right)}} \frac{d \beta(t)}{d t}
$$

Here $\beta(t)$ is the angular distance that the satellite flew in its pathway, and the derivative of $\beta(t)$ will denote the angular velocity of the LEO satellite in the ECF frame, $\omega_{F}(t)$. In other words, we can replace $\frac{d \beta(t)}{d t}$ by using $\omega_{F}(t)$. However, we still need to analyze the expression for $\omega_{F}(t)$, which actually varies due to earth's rotation [12].

The angular velocity of the satellite in the earth centered inertial (ECI) frame, $\omega_{s}(t)$ is constant. $\omega_{E}$ is the angular velocity of the earth's rotation. $i$ denotes the inclination of the orbit. According to [12], the absolute variation of satellite's velocity can be ignored in 
the ECF frame, illustrating the velocity of satellite for the circular orbit can be regarded as constant. Then according to the geometry of the satellite orbit, the relationship between $\omega_{F}(t)$ and $\omega_{S}(t)$ can be approximately equal to:

$$
\omega_{F}(t) \approx \omega_{s}-\omega_{E} \cos i
$$

Finally, the normalized Doppler shift between the LEO satellites and the terminal station can be approximately expressed as:

$$
\frac{\Delta f}{f}=\frac{-R_{E} R \sin \left(\beta(t)-\beta\left(t_{1}\right)\right) \cos \left(\cos ^{-1}\left(\frac{R_{E}}{R} \cos \theta\left(t_{1}\right)\right)-\theta\left(t_{1}\right)\right)}{c \sqrt{\left.R_{E}^{2}+R^{2}-2 R_{E} R \cos \left(\beta(t)-\beta\left(t_{1}\right)\right) \cos \left(\cos ^{-1}\left(\frac{R_{E}}{R} \cos \theta\left(t_{1}\right)\right)-\theta\left(t_{1}\right)\right)\right)}} \omega_{F}(t)
$$

\section{Visibility Window}

Another factor that needs to be considered for communication between a LEO satellite and a terminal station is the visibility window. The prediction for the visibility window also matters since once we know the satellite's visible time from the terminal station, the terminal will be more able to switch between a "working" and a "sleeping" mode, which can conserve power. In addition, the quality of a communication channel has a relationship with the elevation angle of the satellite, the larger the elevation angle of the terminal, the lower probability of line-of-sight blockage. If we choose to transmit more information at a larger elevation angle, the integrity of data can be more guaranteed [13].

One of the efficient ways of deriving the visibility window for a LEO satellite is to express the duration (visibility time) as a function of maximum elevation angle. According to [12] and [13]. The function $\tau\left(\theta_{\max }\right)$ can be approximately analyzed as: 


$$
\begin{aligned}
& \tau\left(\theta_{\text {max }}\right)=2\left(t_{1}-t_{0}\right) \\
& \tau\left(\theta_{\text {max }}\right) \approx \frac{2}{\omega_{s}-\omega_{E} \cos i} \cdot \cos ^{-1}\left(\frac{\cos \left(\cos ^{-1}\left(\frac{R_{E}}{R} \cos \theta\left(t_{0}\right)-\theta\left(t_{0}\right)\right)\right.}{\cos \left(\cos ^{-1}\left(\frac{R_{E}}{R} \cos \theta\left(t_{1}\right)\right)-\theta\left(t_{1}\right)\right)}\right)
\end{aligned}
$$

Where:

$$
\begin{aligned}
& \theta\left(t_{0}\right) \text { the minimum elevation angle of the visibility window } \\
& \theta\left(t_{1}\right) \text { the maximum elevation angle of the visibility window }
\end{aligned}
$$

\subsection{Doppler Frequency Shift for a LEO}

This last section of Chapter 2 will combine the parameters for the Telesat LEO and the Doppler shift equation, in order to make an estimation of how the carrier frequency will change during the transmission between a LEO from Telesat LEO and the terminal station due to the Doppler effect.

Recalling from equation 2.18 , the visibility duration function is:

$$
\tau\left(\theta\left(t_{1}\right)\right) \approx \frac{2}{\omega_{s}-\omega_{E} \cos i} \cdot \cos ^{-1}\left(\frac{\cos \left(\cos ^{-1}\left(\frac{R_{E}}{R} \cos \theta\left(t_{0}\right)-\theta\left(t_{0}\right)\right)\right.}{\cos \left(\cos ^{-1}\left(\frac{R_{E}}{R} \cos \theta\left(t_{1}\right)\right)-\theta\left(t_{1}\right)\right)}\right)
$$

\section{Parameters:}

- $\mathrm{R}_{\mathrm{E}}$ : the radius of the earth: $6.37 \times 10^{6} \mathrm{~m}$

- h: altitude: $1350 \mathrm{~km}$

- $\quad$ R: the radius of Telesat LEO circular orbit: $7.72 \times 10^{6} \mathrm{~m}$

- i: inclination of the orbit: 50 degrees

- $\omega_{E}:$ the angular velocity of the Earth rotation: $7.292115 \times 10^{-5} \mathrm{rad} / \mathrm{s}$ 
- $\omega_{s}:$ the angular velocity of the satellite in the ECI

frame. $\omega_{s}$ can be calculated by using Kepler's constant [14], $(\mu=G M=$

$$
\left.3.986005 \times 10^{14} \mathrm{~m}^{3} / \mathrm{s}^{2}\right): \quad \omega_{s}=\sqrt{\frac{\mu}{R^{3}}}=9.3077 \times 10^{-4} \mathrm{rad} / \mathrm{s}
$$

- $\theta\left(t_{0}\right)$ : the minimum elevation angle from the terminal station observer, here we choose $\theta\left(t_{0}\right)=20$ degrees

- $\theta\left(t_{1}\right)$ : the maximum elevation angle from the terminal station observer

- c: the velocity of electromagnetic wave, here in the vacuo

$$
\mathrm{c}=3 \times 10^{8} \mathrm{~m} / \mathrm{s}
$$

Using the above parameters and the equation for visibility window function, the visibility window duration time can be represented in Figure 2-6:

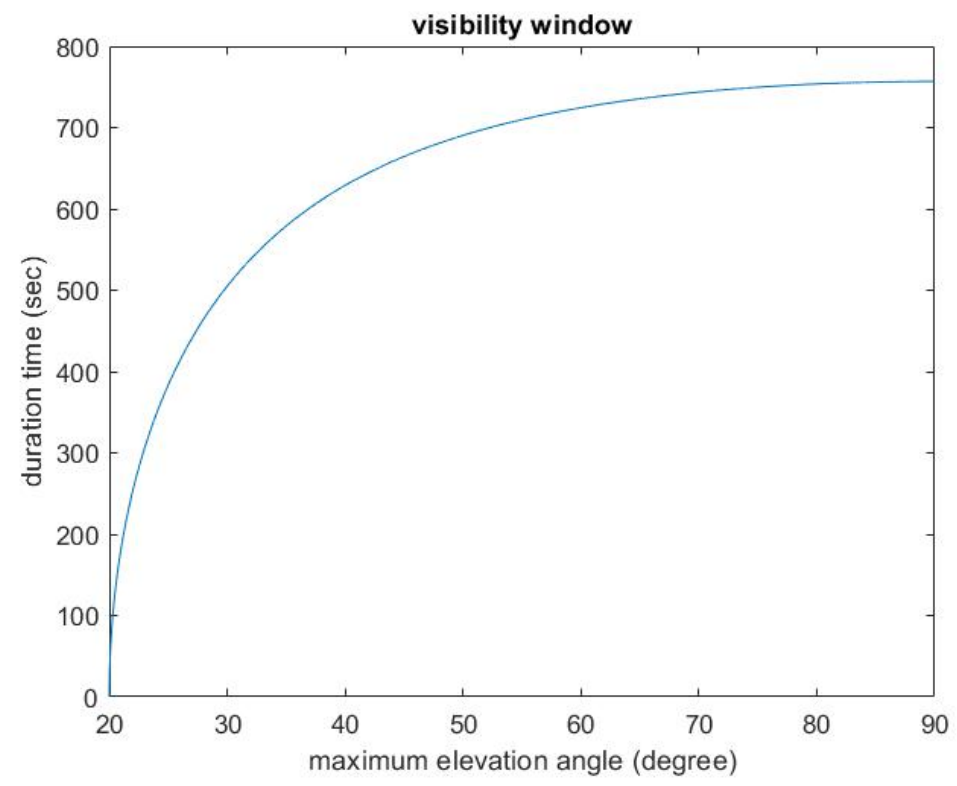

Figure 2-6 Visibility window, the relationship between duration time (t) and the maximum elevation angle (degree) for a LEO satellite in inclined orbit

If we choose a maximum elevation angle $\theta\left(t_{1}\right)=30$ degree, the higher resolution duration time can be seen in Figure 2-7. 


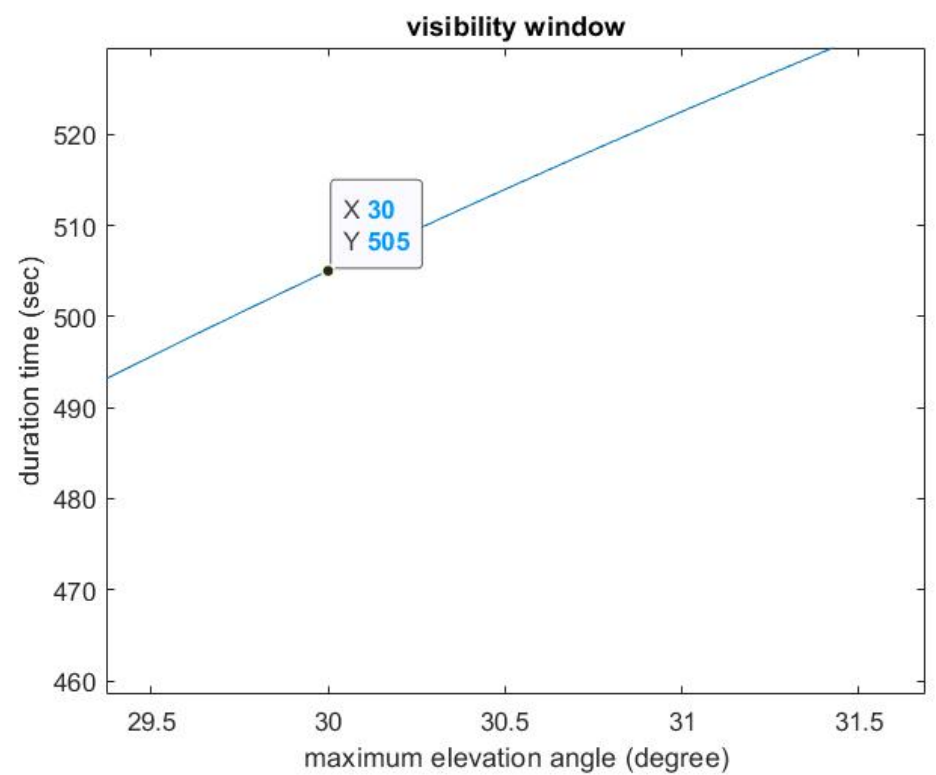

Figure 2-7 The higher resolution duration time for a LEO satellite when the maximum elevation angle is 30 degree

From the figure can we see the duration will be 505 seconds for the communication between a LEO satellite and the terminal station.

Recall the equation 2.16 for normalized Doppler shift equation:

$$
\frac{\Delta f}{f}=\frac{-R_{E} R \sin \left(\beta(t)-\beta\left(t_{1}\right)\right) \cos \left(\cos ^{-1}\left(\frac{R_{E}}{R} \cos \theta\left(t_{1}\right)\right)-\theta\left(t_{1}\right)\right)}{c \sqrt{\left.R_{E}^{2}+R^{2}-2 R_{E} R \cos \left(\beta(t)-\beta\left(t_{1}\right)\right) \cos \left(\cos ^{-1}\left(\frac{R_{E}}{R} \cos \theta\left(t_{1}\right)\right)-\theta\left(t_{1}\right)\right)\right)}} \omega_{F}(t)
$$

Let $\varphi(t)=\beta(t)-\beta\left(t_{1}\right)$. Since $\frac{d \beta(t)}{d t}=\omega_{F}(t)$, from the above analysis in equation 2.15, $\omega_{F}(t) \approx \omega_{s}-\omega_{E} \cos i$, so we can regard $\omega_{F}(t)$ as a constant. Consequently, the expression of $\varphi(t)$ can be written as:

$$
\varphi(\mathrm{t})=\beta(t)-\beta\left(t_{1}\right)=\left(\omega_{s}-\omega_{E} \cos i\right)\left(t-t_{1}\right)
$$




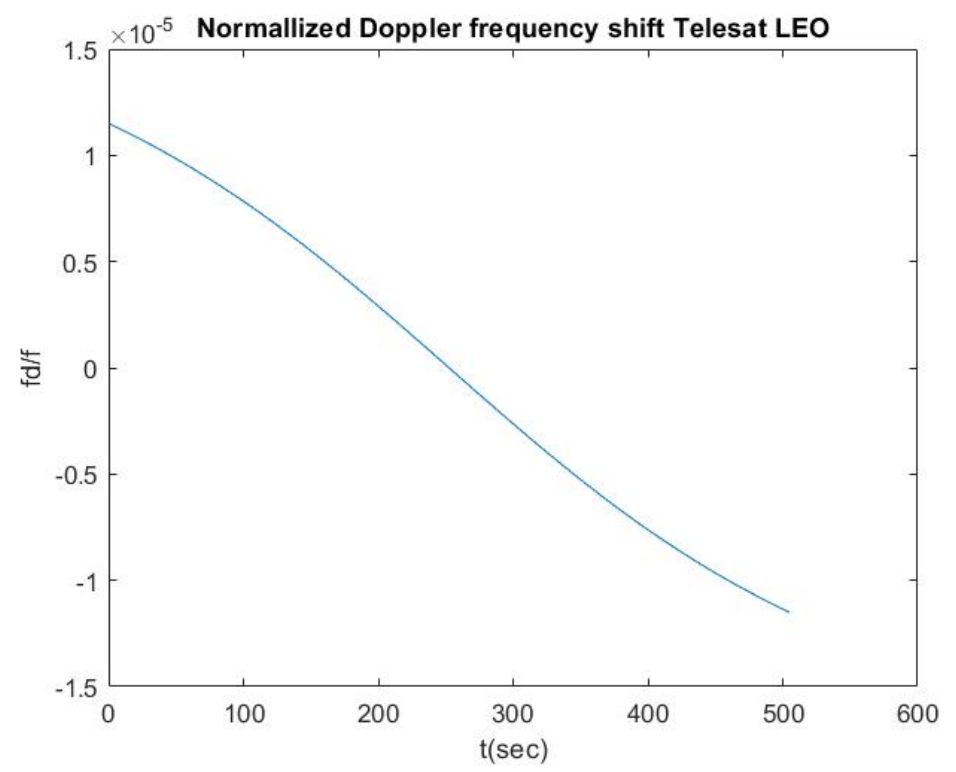

Figure 2-8 The normalized Doppler frequency shift for a LEO satellite in inclined orbit when the maximum elevation angle is 30 degree.

Since the total duration time is 505 seconds and symmetry exists in the visibility window, the instant for maximum elevation angle should be the half of the total duration; as a result, $t_{1}=252.5$ seconds.

Using previous parameters and equation 2.16, the normalized Doppler frequency shift for a LEO satellite and the terminal station can be plotted in Figure 2-8. Since Telesat LEO plans to use a Ka-band spectrum, here I choose $\mathrm{f}$ is equal to $30 \mathrm{GHz}$. From the graph, when $\mathrm{t}=0, \frac{\Delta f}{f}=1.15 \times 10^{-5}$, we can calculate the value of $\Delta f=3.45 \times 10^{5} \mathrm{~Hz}$, at the beginning of the information transition.

\subsection{Summary}

By deriving the function of how Doppler frequency shift occurs when a communication carrier transfers information from a LEO satellite and a terminal station 
and using the parameters provided by the planned, Telesat LEO, this chapter presents the expected duration window and the time function of received frequency offset. When the terminal station receives an incoming signal, the essential task is to demodulate the data from the carrier wave, which requires a PLL to rapidly acquire and track the signals. Precisely, we need to construct a Costas loop to do the phase demodulation and obtain the signals. 


\section{Chapter 3}

\section{BPSK Costas Loop and Natural Acquisition}

In telecommunications, one of the most common ways of modulation is digital phase modulation, also called phase-shift keying. For instance, MPSK provides M possible phases of the carrier that transmits the information [14]. Digital modulation requires PLL to recover the carrier frequency and demodulate the data. There are various circuit designs of PLL to demodulate the data, one of which is the squaring loop. However, since the squaring circuit regenerates the second harmonic of the carrier, which is challenging to realize, most of the circuit designs used to demodulate data are based on the Costas loop $[15]$.

For each category of digital modulation, there are distinct Costas loop designs. The first Costas loop dates back in 1956, which was used to demodulate amplitude-modulated signals with suppressed carrier (DSB-AM). After the invention of phase-shift keying, the Costas loop became essential is primarily applied for tracking and demodulating signals [16].

The most basic phase-shift keying case is binary phase-shift keying (BPSK), meaning it uses two phase offsets to represent the signals. There is only one bit in one symbol duration in BPSK, and the signal can be either 1 or 0 . Figure 3-1 shows the signal space diagram of BPSK. 


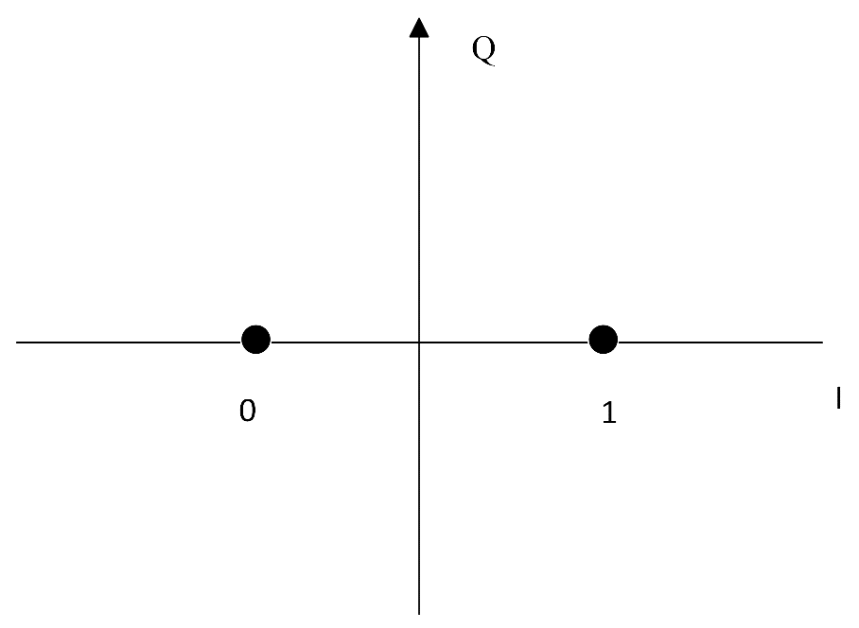

Figure 3-1 BPSK signal space diagram

For convenience, the chapter will use -1 to represent signal 0 of the binary signal.

During a LEO satellite's communication with a ground station, a significant Doppler frequency shift appears while the satellite transfers the information. Hence, there is a need to construct a Costas loop to track the change of frequency and demodulate the transferred data.

In a PLL, when a signal comes into the loop, there will be an unknown phase or frequency difference occurring between VCO and the input signal. Considering the complexity of the phase detector and characteristics of the loop filter and VCO, there are nonlinear equations describing the loop performance. This results in the complicatedness of the analysis of the PLL [17]. Likewise, in the initial case of the LEO satellite's wireless communication, because of the sudden appearance of the LEO satellites and the Doppler frequency offset, it is essential to analyze of the natural acquisition for the Costas loop. 


\subsection{Natural acquisition for BPSK Costas loop with 1st order}

\section{filter}

Let us consider the simple one, BPSK Costas loop with first order filter, where $\mathrm{F}(\mathrm{s})=1$.

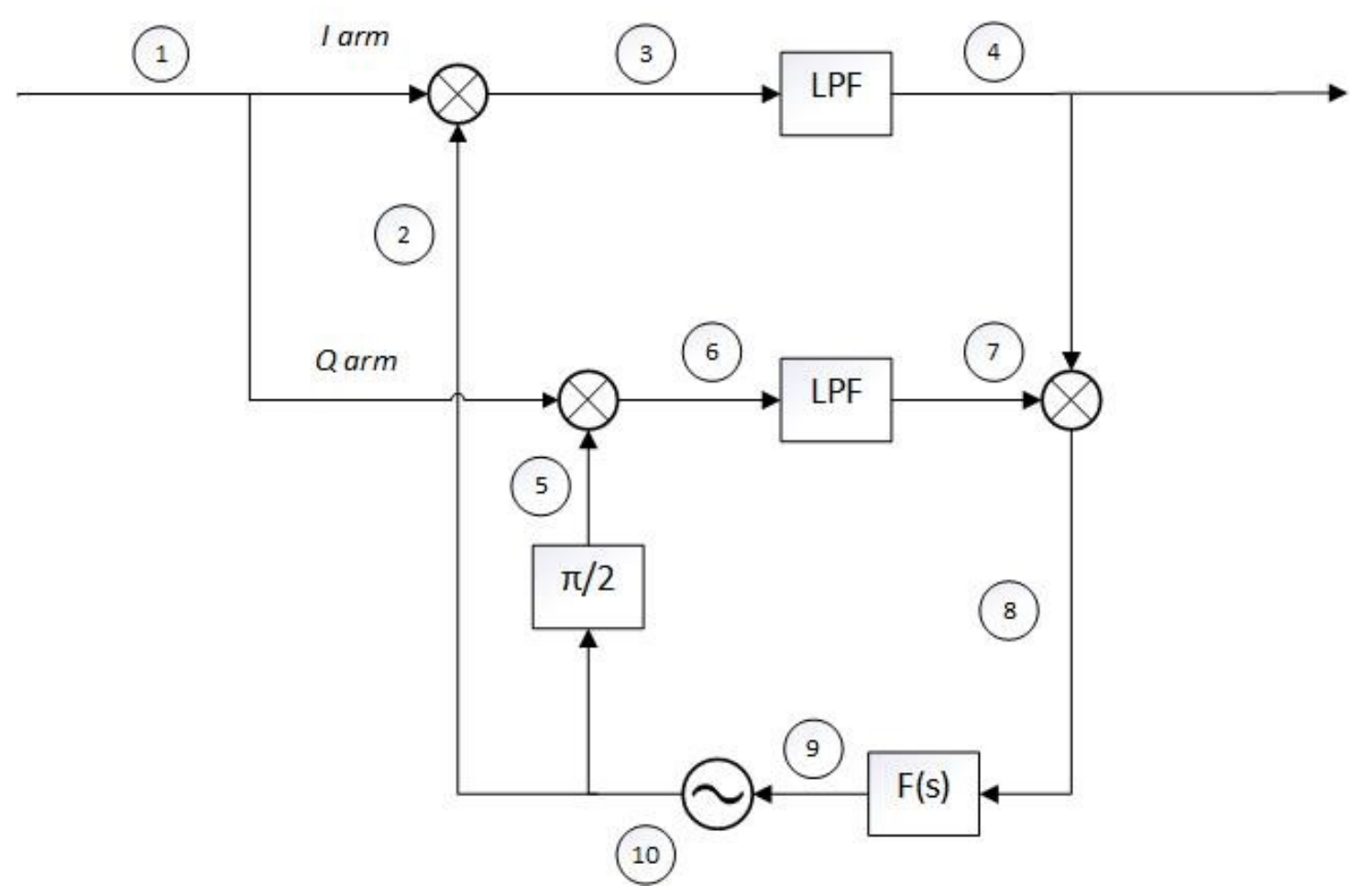

Figure 3-2 BPSK Costas loop

As shown in Figure 3-2, the input signal is $m(t) \sin \left(\omega_{i} t+\theta_{i}\right)$, (shown in (1)) where $m(t)$ is the BPSK transmitted data and $\omega_{i}$ is the carrier frequency. The initial output of the VCO (shown in (2)) can be chosen as $2 \sin \left(\omega_{o} t+\theta_{o}\right)$.

In the I arm side, the output (shown in (3)) will be the value multiplied by the two input values, and then after being filtered by the LPF (low pass filter), the high frequency 
will be removed. Consequently, the signal at (4) will be $m(t) \cos \left[\left(\omega_{i}-\omega_{o}\right) t+\left(\theta_{i}-\right.\right.$ $\left.\left.\theta_{o}\right)\right]$

In the $Q \mathrm{arm}$, there is phase shift of $\frac{\pi}{2}$ of the output of the VCO, making (5) into $2 \cos \left(\omega_{o} t+\theta_{o}\right)$. As for the $I$ arm, after passing through LPF, the output will be $m(t) \sin \left[\left(\omega_{i}-\omega_{o}\right) t+\left(\theta_{i}-\theta_{o}\right)\right]$.

The output of both $I$ arm and $Q$ arm are then multiplied together, producing a new signal, $\frac{1}{2} m^{2}(t) \sin \left[2\left(\omega_{i}-\omega_{o}\right) t+2\left(\theta_{i}-\theta_{o}\right)\right]$, which has a double frequency as the $I$ or $Q$ arm (shown in (8)), since $\mathrm{F}(\mathrm{s})=1$, the signal in (9) will be the same as (8).

In fact, the phase of the output of the VCO is a function of time. In other words, $\theta_{o}=\phi_{o}(t)$. According to the characteristic of the $\mathrm{VCO}$, the function can be shown as:

$$
\frac{d \phi_{o}}{d t}=K_{3} u_{2}=\frac{K_{3}}{2} m^{2}(t) \sin \left[2\left(\omega_{i}-\omega_{o}\right) t+2\left(\theta_{i}-\phi_{o}(t)\right)\right.
$$

(where $\mathrm{K}_{3}$ is the modulation sensitivity of the $\mathrm{VCO}$ with the unit of $\mathrm{rad} / \mathrm{s} / \mathrm{v}$ )

$$
\text { Let } \phi(t)=\left(\omega_{i}-\omega_{o}\right) t+\theta_{i}-\phi_{o}(t)=\Omega_{0} t+\theta_{i}-\phi_{o}(t)
$$

Differentiating the equation 3.2 on both sides, then we can get:

$$
\begin{gathered}
\frac{d \phi(t)}{d t}=\Omega_{0}-\frac{d \phi_{o}(t)}{d t}=\Omega_{0}-K \sin (2 \phi) \\
\left(K \text { is equal to } \frac{K_{3}}{2} m^{2}(t)\right)
\end{gathered}
$$

The following analysis will be performed on this equation.

\section{Phase acquisition:}

To get phase acquisition, the case can be simplified into the condition of $\Omega_{0}=0$, then the equation will be: 


$$
\frac{d \phi(t)}{d t}=-K \sin (2 \phi)
$$

After integration, the equation 3.4 will become $\frac{1}{2} \ln (\tan \phi)=-K\left(t-t_{0}\right)$,

When $\mathrm{t}=0, \phi=\theta_{i}$, we can get $t_{0}=\frac{\ln \left(\tan \theta_{i}\right)}{K}$, then the equation will become:

$\frac{1}{2} \ln (\tan \phi)=-K t+\frac{1}{2} \ln \left(\tan \theta_{i}\right)$ from which we can conclude that:

$$
\phi=\tan ^{-1}\left(e^{-2 K t} \cdot \tan \theta_{i}\right)
$$

The situation can be classified in two cases according to the characteristic of K.

1) If $\mathrm{K}>0$, as $\mathrm{t}$ increases, $\boldsymbol{e}^{-\mathbf{2 K t}} \cdot \boldsymbol{t a n} \boldsymbol{\theta}_{\boldsymbol{i}}$ decreases and tends to be 0 , causing $\phi$ to be 0

2) If $\mathrm{K}<0$, as $\mathrm{t}$ increases, $\boldsymbol{e}^{-\mathbf{2 K t}} \cdot \boldsymbol{t a n} \boldsymbol{\theta}_{\boldsymbol{i}}$ increases and tends to be $\infty$, causing $\phi$ to be $\frac{\pi}{2}$

The figures can be shown in MATLAB (Figure 3-3, 3-4), if we assume $\left|K_{3}\right|=$ $10^{6}, m^{2}(t)=1$, then $|K|=5 \times 10^{5}$, the initial input phase value, $\theta_{i}=\frac{\pi}{3}$.

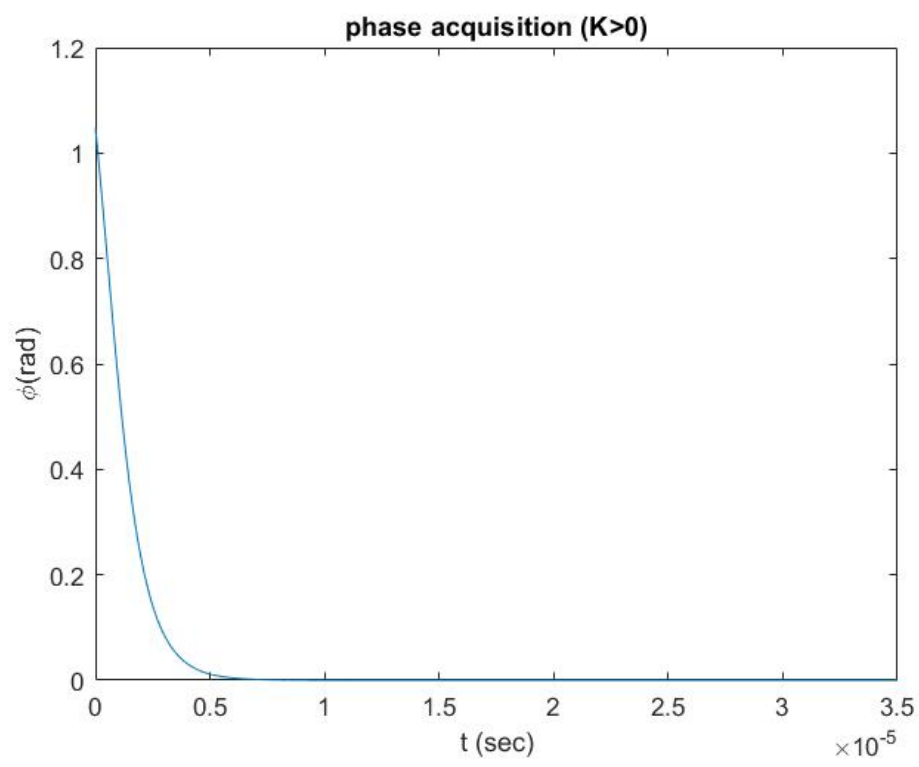

Figure 3-3 Phase acquisition when $K>0$ 


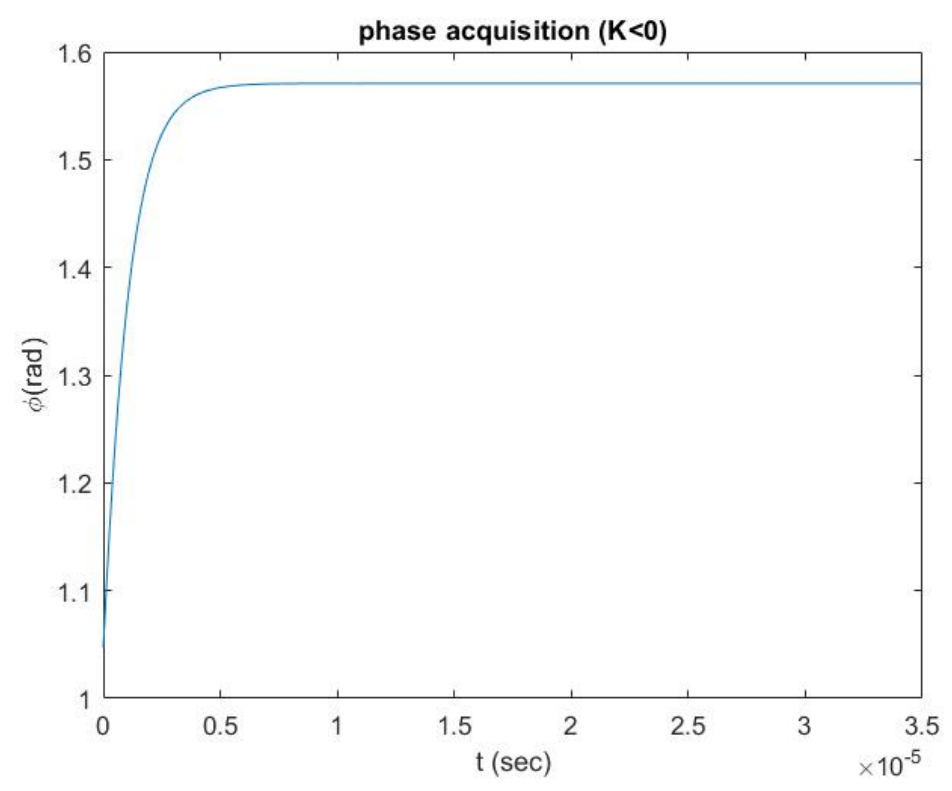

Figure 3-4 Phase acquisition when $K<0$

If $\mathrm{K}>0$, when the phase achieves synchronization, $\phi$ is 0 , which means there is no phase difference between the VCO and input signal (as shown in Figure 3-2) If $\mathrm{K}<0$, when the phase achieves synchronization, $\phi$ will be $\frac{\pi}{2}$, meaning a lag of the VCO by $90^{\circ}$ with regard to the incoming signal as shown in Figure 3-4.

\section{Frequency acquisition}

This time the initial frequency difference, $\Omega_{0}$, is not equal to 0 ; consequently, the equation becomes

$$
\frac{d \phi(t)}{d t}=\Omega_{0}-K \sin (2 \phi)
$$

To analyze the equation, we need to make a comparison between the value of $\Omega_{0}$ and $K$. 
1) $\left|\Omega_{0}\right|>|K|$

After integration, from equation 3.6, the function can be simplified into:

$$
t-t_{0}=\frac{1}{\sqrt{\Omega_{0}{ }^{2}-K^{2}}} \tan ^{-1}\left(\frac{\Omega_{0} \tan (\phi)-K}{\sqrt{\Omega_{0}{ }^{2}-K^{2}}}\right)
$$

From which we can derive that

$$
\phi(t)=\tan ^{-1}\left\{\frac{1}{\Omega_{0}}\left[\sqrt{\Omega_{0}^{2}-K^{2}} \tan \left[\left(t-t_{0}\right) \sqrt{\Omega_{0}^{2}-K^{2}}\right]+K\right]\right\}
$$

The function $\phi(t)$ this time becomes periodic. By using MATLAB, we can draw the shape of the function which is shown in figure 3-5 by choosing the parameter $\Omega_{0}=500$ $\mathrm{krad} / \mathrm{s}$, and $K=400 \mathrm{kad} / \mathrm{v}$.

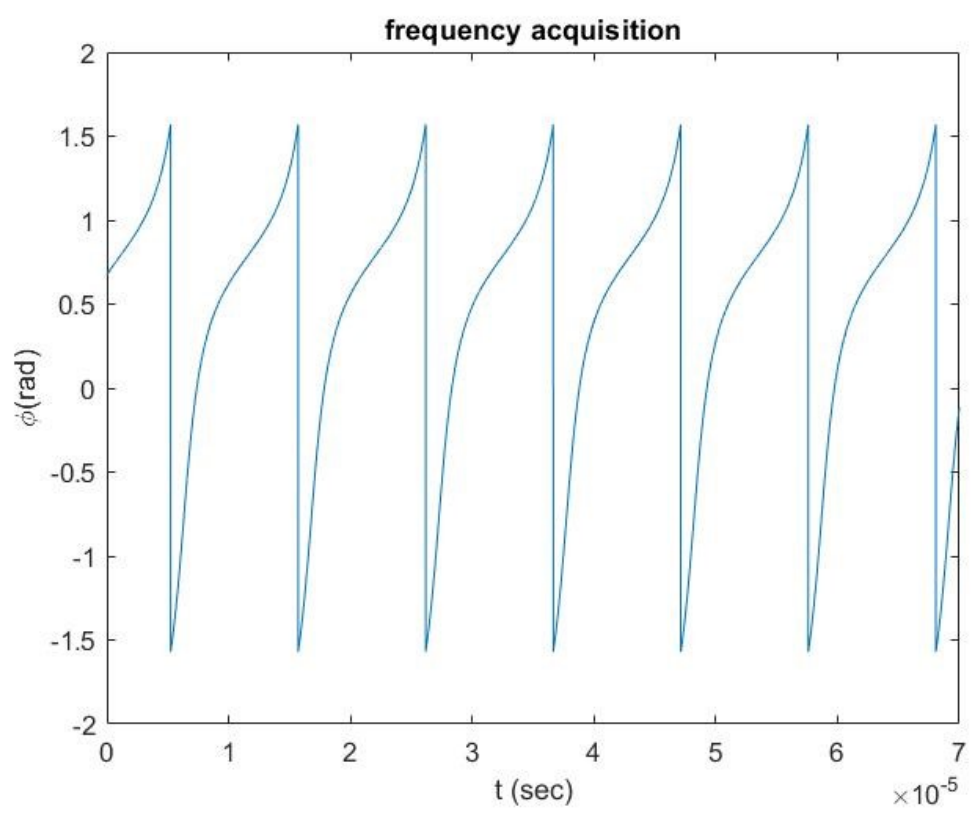

Figure 3-5 Frequency acquisition when $\Omega_{0}>K$

From the plot can we see that the phase changes periodically; in other words, there is no steady state solution for $\left|\Omega_{0}\right|>|K|$. 
2) $\left|\Omega_{0}\right|<|K|$

Under the condition of the amplitude of initial frequency difference is smaller than $K$, it is necessary to distinguish the condition into two parts.

Case 1: When $\sqrt{K^{2}-\Omega_{0}^{2}}>\left|K-\Omega_{0}\right| \tan (\phi)$

After integrating equation 3.6, the function becomes:

$$
t-t_{0}=\frac{1}{2 \sqrt{\Omega_{0}{ }^{2}-K^{2}}} \ln \frac{\sqrt{K^{2}-\Omega_{0}^{2}}+\left(K-\Omega_{0} \tan (\phi)\right)}{\sqrt{K^{2}-\Omega_{0}^{2}}-\left(K+\Omega_{0} \tan (\phi)\right)}
$$

From equation $3.9, \tan (\phi)$ can be derived that

$$
\tan (\phi)=\frac{K}{\Omega_{0}}-\frac{\sqrt{K^{2}-\Omega_{0}^{2}}}{\Omega_{0}}\left(\frac{\exp \left(2 \sqrt{K^{2}-\Omega_{0}^{2}}\right)\left(t-t_{0}\right)-1}{\exp \left(2 \sqrt{K^{2}-\Omega_{0}^{2}}\right)\left(t-t_{0}\right)+1}\right)
$$

To get $t_{0}$ in the equation 3.10 , the assumption of the initial case is that when $t=0$, $\phi=\theta_{i}$. Substituting back into the equation 3.9, using the value of $\phi$ when $\mathrm{t}$ is equal to 0 $\mathrm{t}_{0}$ can be attained in equation 3.11 :

$$
-t_{0}=\frac{1}{2 \sqrt{\Omega_{0}{ }^{2}-K^{2}}} \ln \frac{\sqrt{K^{2}-\Omega_{0}{ }^{2}}+\left(K-\Omega_{0} \tan \left(\theta_{i}\right)\right)}{\sqrt{K^{2}-\Omega_{0}{ }^{2}}-\left(K+\Omega_{0} \tan \left(\theta_{i}\right)\right)}
$$

Then replacing the value of $t_{0}$, using a further simplification of the equation (3.10), the result will become:

$$
\tan (\phi)=\frac{K}{\Omega_{0}}-\frac{\sqrt{K^{2}-\Omega_{0}^{2}}}{\Omega_{0}}\left(\frac{C \cdot \exp \left(2 \sqrt{K^{2}-\Omega_{0}^{2}}\right) t-1}{C \cdot \exp \left(2 \sqrt{K^{2}-\Omega_{0}^{2}}\right) t+1}\right)
$$


Where:

$$
C=\frac{\sqrt{K^{2}-\Omega_{0}^{2}}+\left(K-\Omega_{0} \tan \left(\theta_{i}\right)\right)}{\sqrt{K^{2}-\Omega_{0}^{2}}-\left(K+\Omega_{0} \tan \left(\theta_{i}\right)\right)}
$$

Case 2: When $\sqrt{K^{2}-\Omega_{0}{ }^{2}}>\left|K-\Omega_{0}\right| \tan (\phi)$

Under the case of $\sqrt{K^{2}-\Omega_{0}^{2}}>\left|K-\Omega_{0}\right| \tan (\phi)$, there is a small difference of integration 3.12, compared with equation 3.9.

$$
t-t_{0}=\frac{1}{2 \sqrt{\Omega_{0}{ }^{2}-K^{2}}} \ln \frac{\left(K-\Omega_{0} \tan (\phi)\right)+\sqrt{K^{2}-\Omega_{0}{ }^{2}}}{\left(K-\Omega_{0} \tan (\phi)\right)-\sqrt{K^{2}-\Omega_{0}{ }^{2}}}
$$

Likewise, using the same method as the above, the values will be as follows:

$$
\begin{aligned}
\tan (\phi) & =\frac{K}{\Omega_{0}}-\frac{\sqrt{K^{2}-\Omega_{0}{ }^{2}}}{\Omega_{0}}\left(\frac{\exp \left(2 \sqrt{K^{2}-\Omega_{0}{ }^{2}}\right)\left(t-t_{0}\right)+1}{\exp \left(2 \sqrt{K^{2}-\Omega_{0}{ }^{2}}\right)\left(t-t_{0}\right)-1}\right) \\
-t_{0} & =\frac{1}{2 \sqrt{\Omega_{0}{ }^{2}-K^{2}}} \ln \frac{\left(K-\Omega_{0} \tan \left(\theta_{i}\right)\right)+\sqrt{K^{2}-\Omega_{0}{ }^{2}}}{\left(K-\Omega_{0} \tan \left(\theta_{i}\right)\right)-\sqrt{K^{2}-\Omega_{0}{ }^{2}}}
\end{aligned}
$$

Putting the value of $t_{0}(3.15)$ back into (3.14), the result for tan $(\phi)$ will be the same as previous condition (3.12). As a result, the function of $\phi$ will be:

$$
\phi=\tan ^{-1}\left(\frac{K}{\Omega_{0}}-\frac{\sqrt{K^{2}-\Omega_{0}^{2}}}{\Omega_{0}}\left(\frac{C \cdot \exp \left(2 \sqrt{K^{2}-\Omega_{0}^{2}}\right) t-1}{C \cdot \exp \left(2 \sqrt{K^{2}-\Omega_{0}^{2}}\right) t+1}\right)\right)
$$


Where:

$$
C=\frac{\sqrt{K^{2}-\Omega_{0}^{2}}+\left(K-\Omega_{0} \tan \left(\theta_{i}\right)\right)}{\sqrt{K^{2}-\Omega_{0}{ }^{2}}-\left(K+\Omega_{0} \tan \left(\theta_{i}\right)\right)}
$$

\section{Analysis for equation 3.16:}

In the equation 3.16 , when the time, $t$, increases to be infinite, the phase, $\phi$ will tend to a specific value.

$$
\phi_{\infty}=\tan ^{-1}\left(\frac{K}{\Omega_{0}}-\frac{\sqrt{K^{2}-\Omega_{0}^{2}}}{\Omega_{0}}\right)
$$

The following figures show the relationship between the time and the change of the phase in the MATLAB.

$\left(\mathrm{K}=500 \mathrm{krad} / \mathrm{s}, \Omega_{0}=400 \mathrm{krad} / \mathrm{s}\right.$, as a result, $\left.\phi_{\infty}=0.464 \mathrm{rad}\right)$

- Case 1: $-\frac{\pi}{2}<\theta_{i}<\phi_{\infty}$

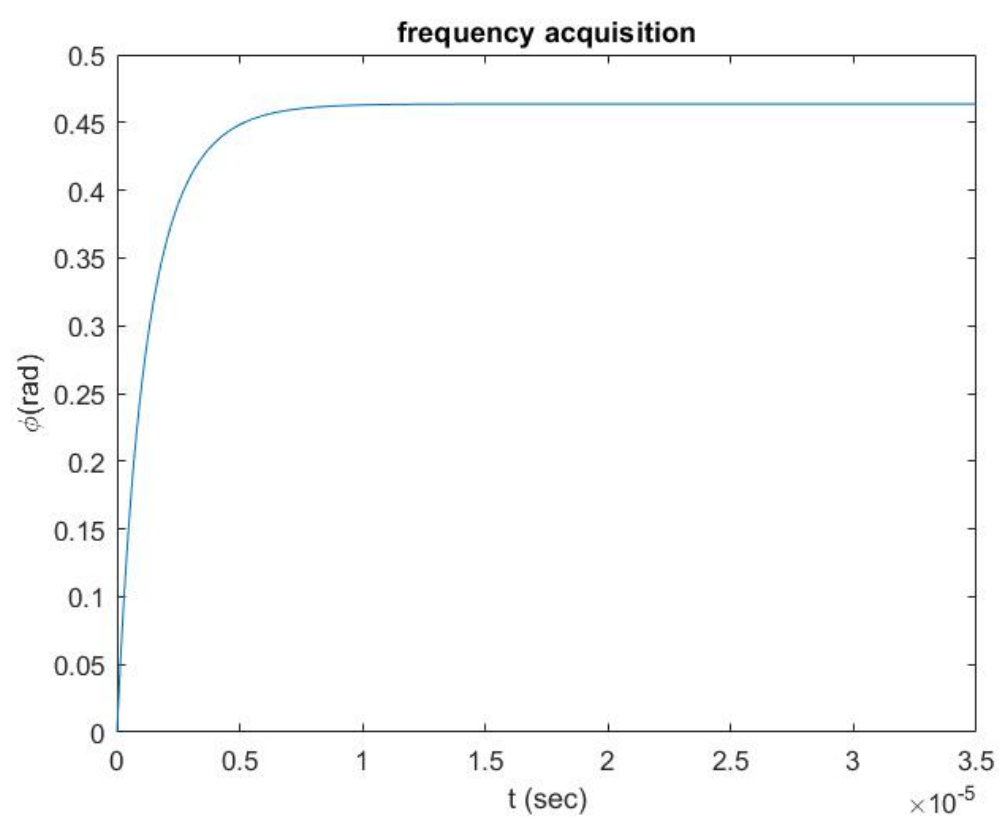

Figure 3-6 Frequency acquisition when $-\frac{\pi}{2}<\theta_{i}<\phi_{\infty}$ 
- Case 2: $\phi_{\infty}<\theta_{i}<\frac{\pi}{2}-\phi_{\infty}$

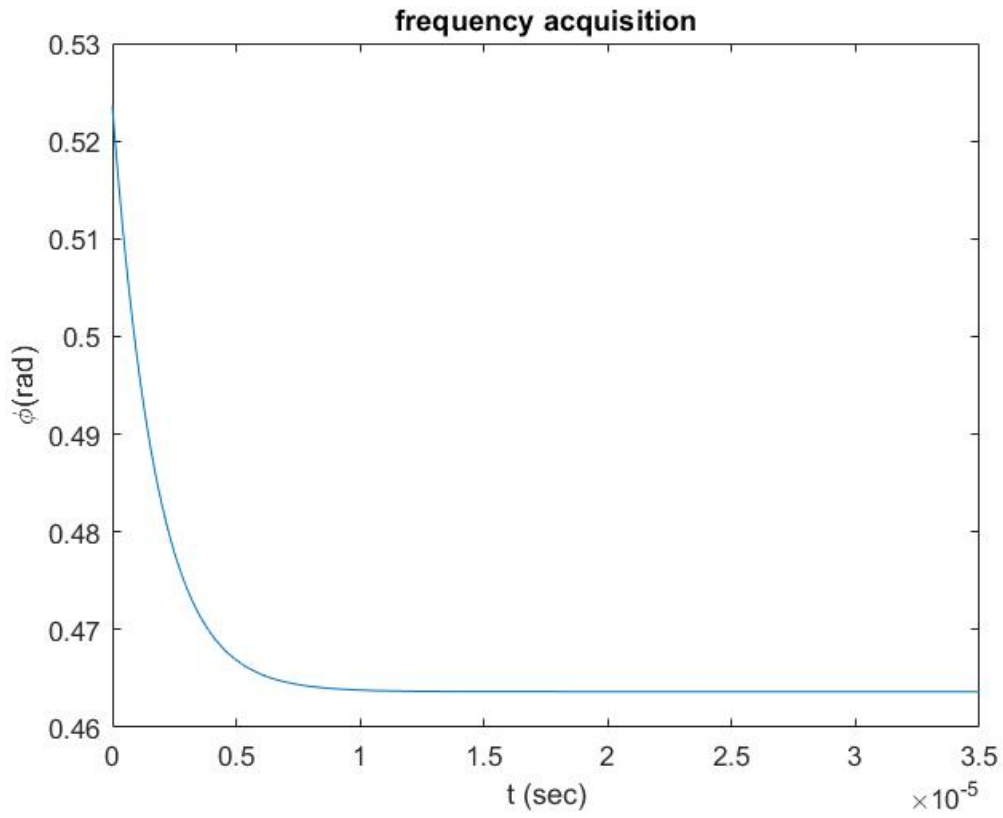

Figure 3-7 Frequency acquisition when $\phi_{\infty}<\theta_{i}<\frac{\pi}{2}-\phi_{\infty}$

- Case 3: $\frac{\pi}{2}-\phi_{\infty}<\theta_{i}<\frac{\pi}{2}$

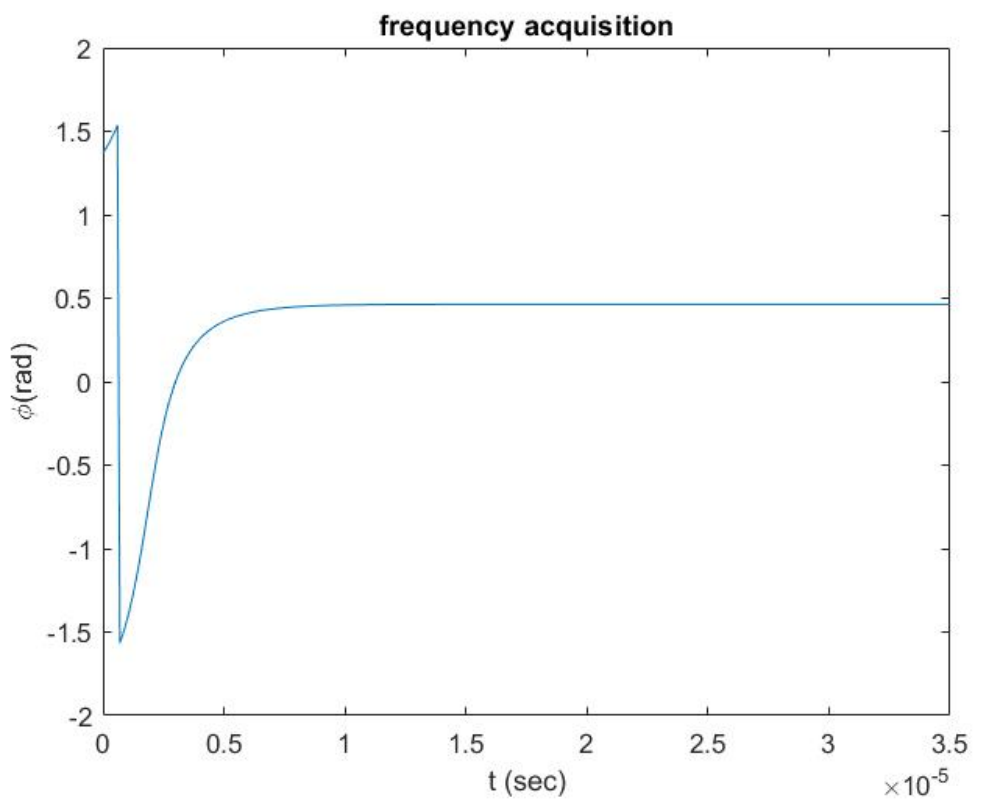

Figure 3-8 Frequency acquisition when $\frac{\pi}{2}-\phi_{\infty}<\theta_{i}<\frac{\pi}{2}$ 
Then, define $u_{1}=\cos \phi$, we can get the relationship between the time, $\mathrm{t}$, and the signal $\mathrm{u}_{1}$ (the value of $\mathrm{K}_{1}$ here is $1 \mathrm{~V} / \mathrm{rad}$ )

- $\quad$ Case 1: $-\frac{\pi}{2}<\theta_{i}<0$

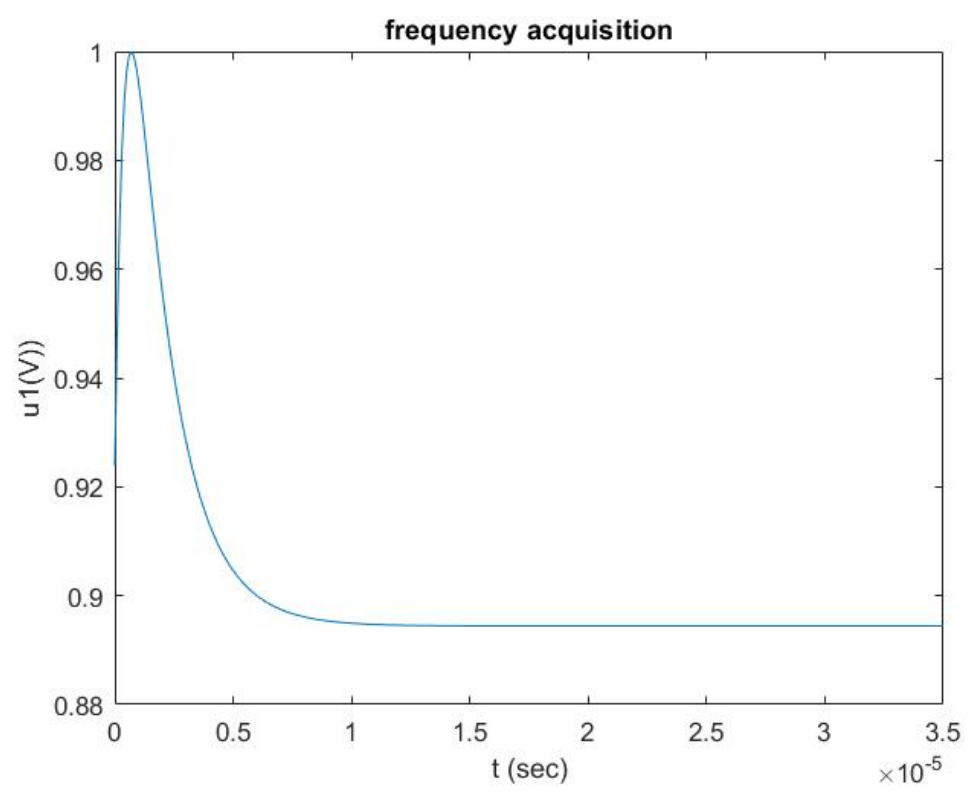

Figure 3-9 Signal u1 when $-\frac{\pi}{2}<\theta_{i}<0$

- $\quad$ Case 2: $0<\theta_{i}<\phi_{\infty}$

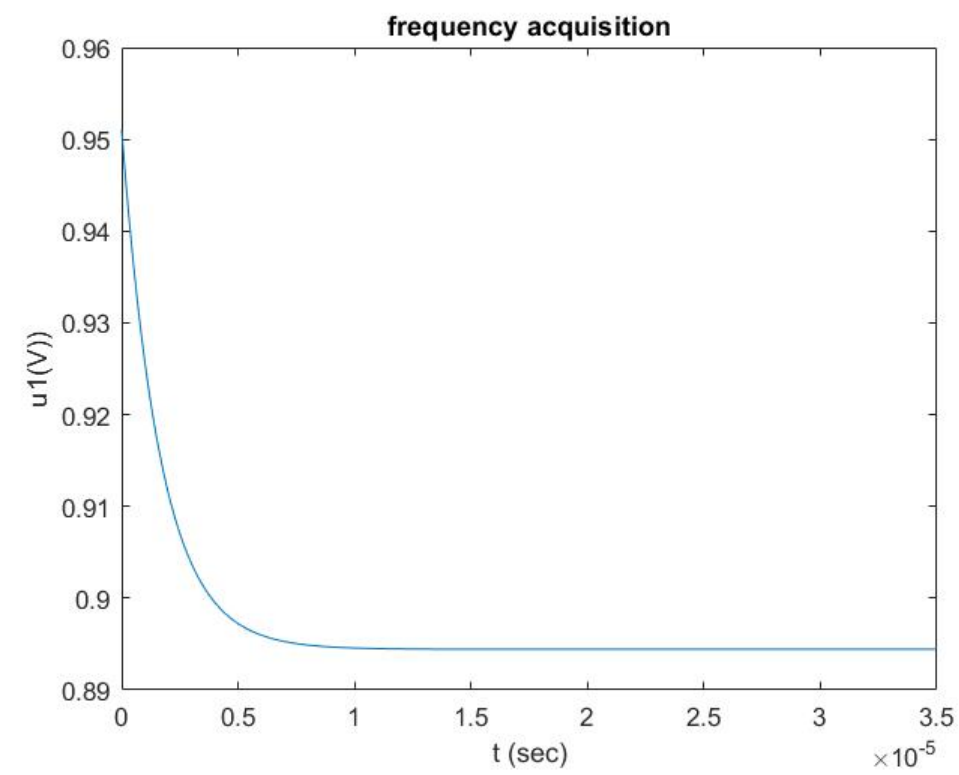

Figure 3-10 Signal u1 when $0<\theta_{i}<\phi_{\infty}$ 
- Case 3: $\phi_{\infty}<\theta_{i}<\frac{\pi}{2}-\phi_{\infty}$

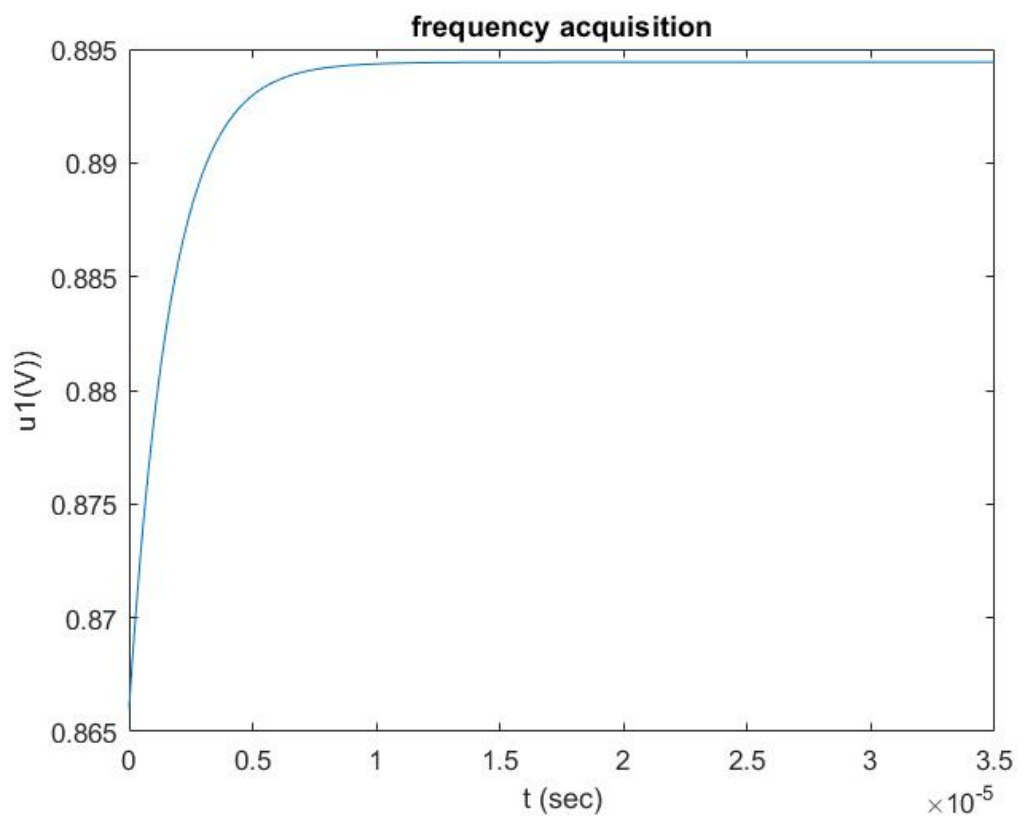

Figure 3-11 Signal u1 when $\phi_{\infty}<\theta_{i}<\frac{\pi}{2}-\phi_{\infty}$

- Case 4: $\frac{\pi}{2}-\phi_{\infty}<\theta_{i}<\frac{\pi}{2}$

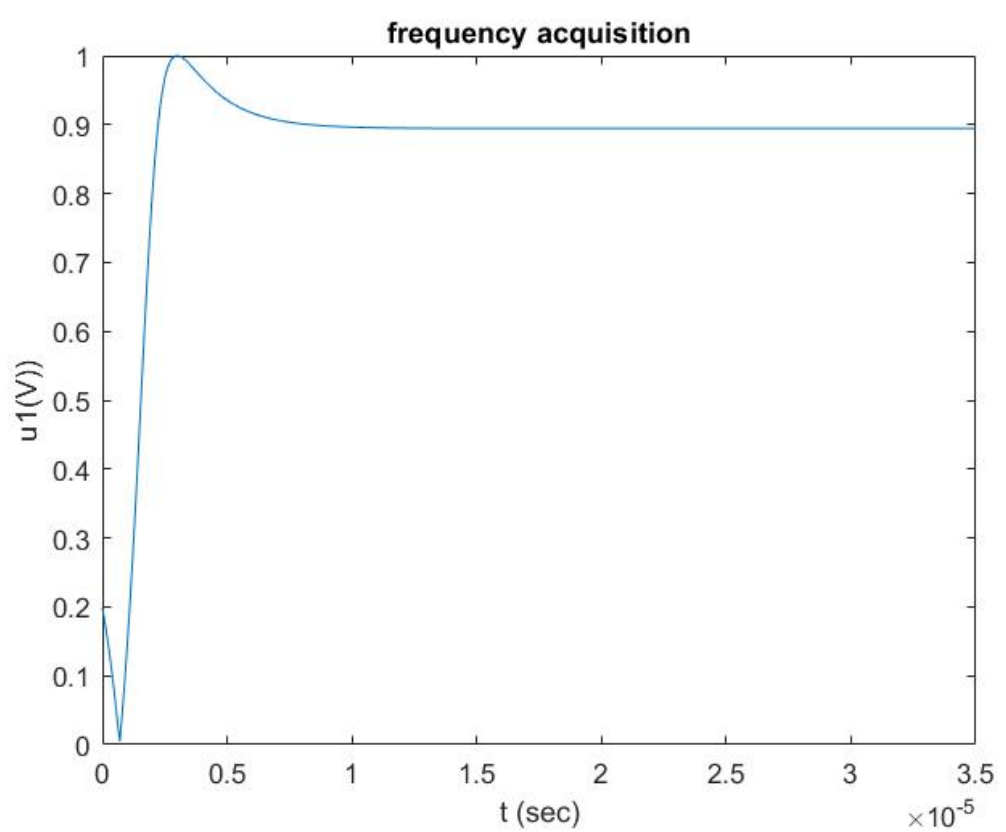

Figure 3-12 Signal u1 when $\frac{\pi}{2}-\phi_{\infty}<\theta_{i}<\frac{\pi}{2}$ 


\subsection{Loop filter}

The above theoretical analysis is under the condition of $F(s)=1$, however, in the actual design of PLL, there is a need of the loop filter before the VCO to modulate the signal and achieve synchronization.

One of the most common filters used in a second order loop is the integrator with phase-lead correction. The circuit of filter is shown in Figure 3-13:

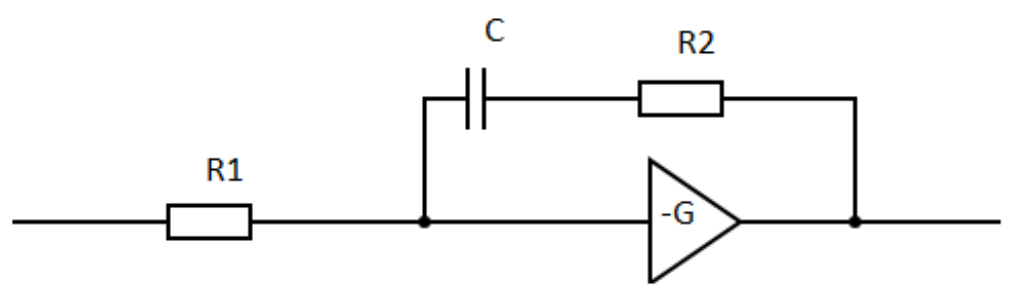

Figure 3-13 The circuit structure of integrator with phase-lead correction

The approximate function of integrator with phase lead correction is [17]:

$$
F(s)=\frac{1+\tau_{2} s}{\tau_{1} s}
$$

where $\tau_{1}=R_{1} C, \quad \tau_{2}=R_{2} C$

here we still have:

$$
\begin{gathered}
\omega_{n}^{2}=\frac{K}{\tau_{1}} \\
2 \zeta \omega_{n}=\frac{K \tau_{2}}{\tau_{1}}
\end{gathered}
$$

Where

$$
\omega_{n} \text { : natural frequency }
$$


$K$ : synchronization bandwidth

$\zeta$ : damping constant

\section{Character of the loop filter (Integrator with phase lead correction):}

- Stability

To analyze the stability of the filter, one of the most useful ways is to present it in Bode plot.

In PLL, the open-loop transfer function is:

$$
G(s)=K \frac{F(s)}{s}=K \frac{1+\tau_{2} s}{\tau_{1} s^{2}}
$$

Where $\mathrm{K}$ is equal to $\mathrm{K}_{1} \mathrm{~K}_{3}, \mathrm{~K}_{1}$ denotes the sensitivity of the phase detector with the unit of $\mathrm{V} / \mathrm{rad}$. Then the Bode diagram of $G(s)$ can be represented in Figure 3-14.

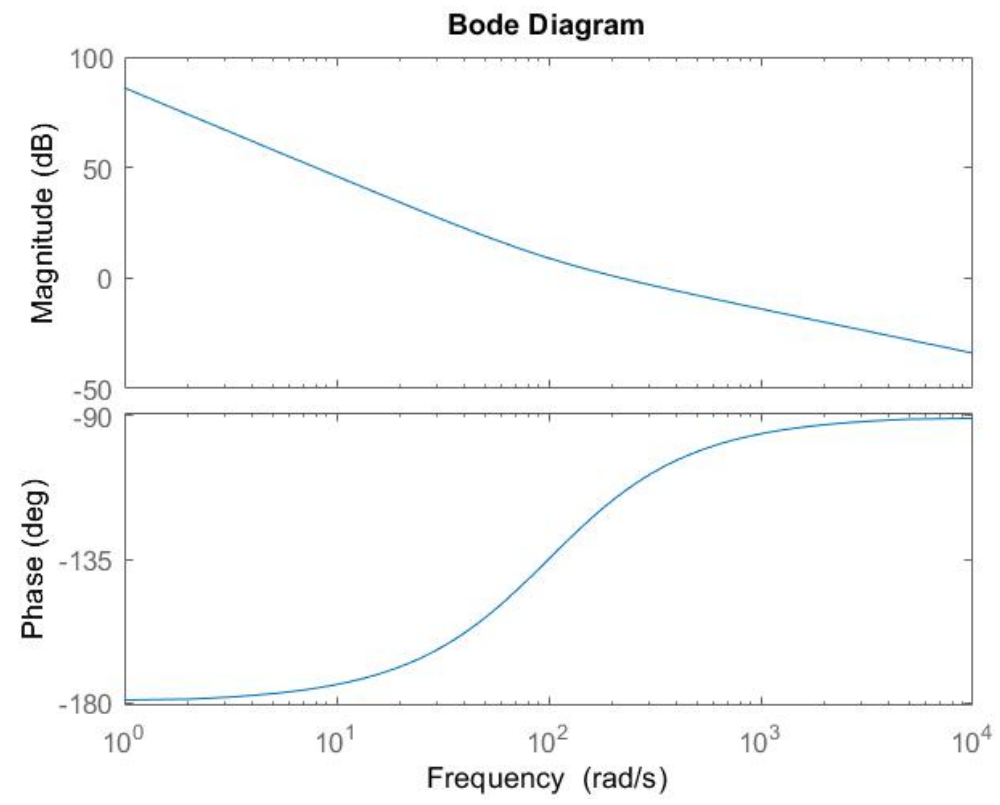

Figure 3-14 Bode plot for integrator with phase-lead correction 
The details can be shown in Figure 3-15:
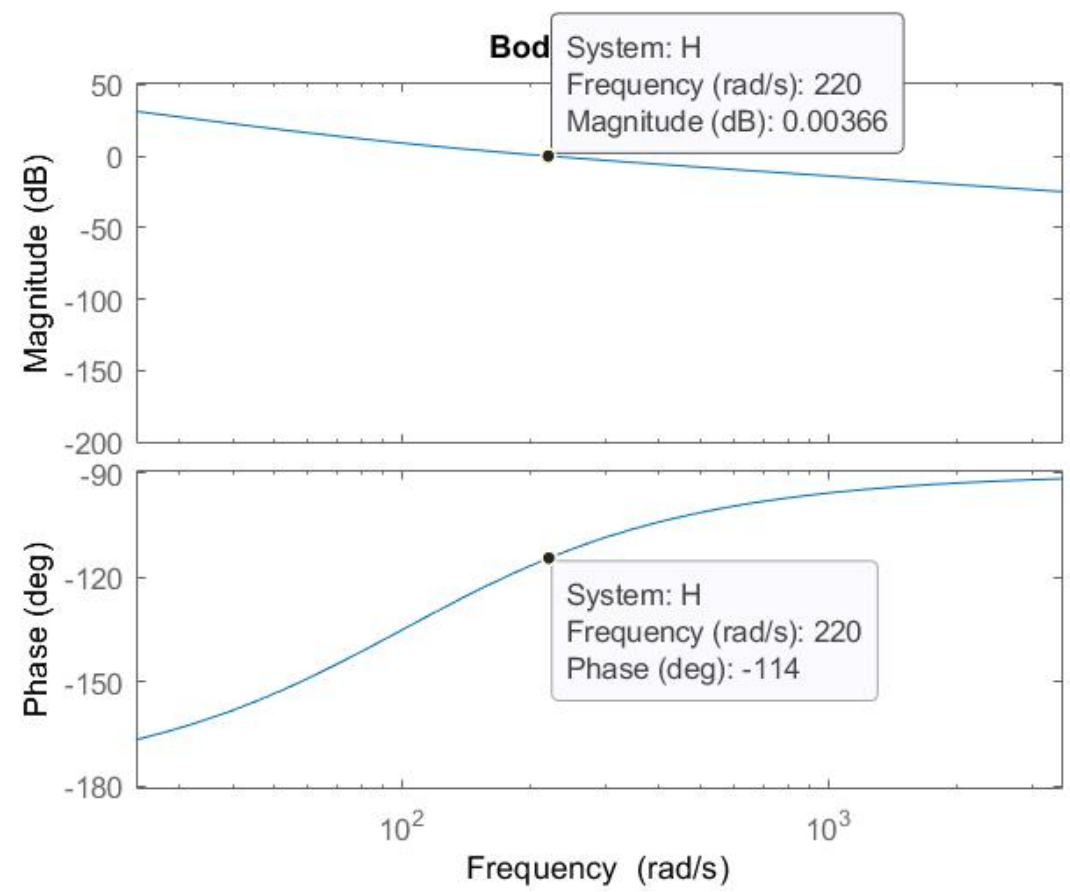

Figure 3-15 The phase degree for Bode plot for integrator with phase lead correction when magnitude is zero

The example shown in Figure 3-15 has good stability since when the Magnitude $\mathrm{G}(\mathrm{s})=0 \mathrm{~dB}$, the phase shift is -114 degree, far from -180 degree, indicating that the loop is in stability. In fact, if we want the loop to have good stability, the condition to satisfy is:

$$
\omega_{n} \tau_{2}>1
$$

Combing equations 3.19 and 3.20, the condition for good stability of the loop filter can be concluded as:

$$
\zeta>\frac{1}{2}
$$

The Bode plot of different values of $\zeta$ are compared in Figure 3-16: 


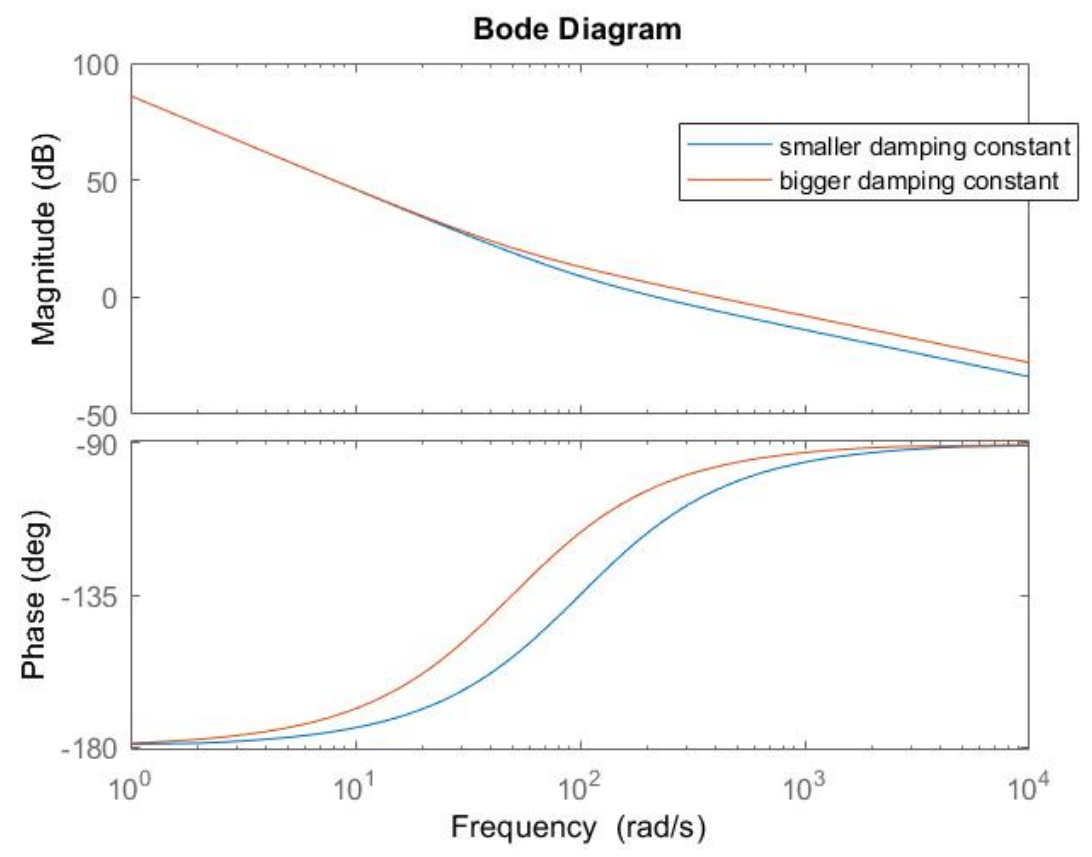

Figure 3-16 Comparison between the two Bode plots with two different values of damping constant $\zeta$

When the value of the damping constant $\zeta$ increases, the phase shift is farther away from the -180 degree, giving better stability for the loop.

\section{- Transient response}

Apart from the stability, another import factor to be considered is how PLL reacts for the input. In other words, when an unknown signal comes into the loop, we should analyze the transient response of the PLL to see how the parameters of the loop filter can influence the speed for response.

Since there is a sudden frequency change due to Doppler shift in the transmission when the terminal station first observes a LEO satellite, an optional way of testing the transient response is to use a frequency step signal. 
A simple way of testing the response is to use general linearized equations [17], in other words, if the difference between $\phi_{i}(t)$, the phase function of the input, and $\phi_{o}(t)$, the phase function of the output of VCO is small, $<0.5 \mathrm{rad}$, the equation can be approximately as:

$$
\sin \left(\phi_{i}(t)-\phi_{o}(t)\right) \approx \phi_{i}(t)-\phi_{o}(t)
$$

In the character of basic PLL, the relationship among the VCO, loop filter and error signals can be also stated in the time domain as:

$$
\frac{d \phi_{o}(t)}{d t}=K\left[\phi_{i}(t)-\phi_{o}(t)\right] * f(t)
$$

To analyze in an easier way, taking the Laplace transform, the equation can be stated in the frequency domain as:

$$
s \phi_{o}(s)=K\left[\phi_{i}(s)-\phi_{o}(s)\right] F(s)
$$

The instantaneous phase error is:

$$
\begin{aligned}
\phi(t) & =\phi_{i}(t)-\phi_{o}(t) \\
\text { so: } \phi(s) & =\phi_{i}(s)-\phi_{o}(s)
\end{aligned}
$$

Combining equation 3.25 and 3.27 , the relationship between the error signal and input signal in the frequency domain can be presented as:

$$
\frac{\phi(s)}{\phi_{i}(s)}=\frac{s}{s+K F(s)}
$$

Recall the function of the second-order loop filter, the integrator with phase lead correction, equation 3.18 :

$$
F(s)=\frac{1+\tau_{2} s}{\tau_{1} s}
$$

Equation 3.28 can be further represented as: 


$$
\frac{\phi(s)}{\phi_{i}(s)}=\frac{\tau_{1} s^{2}}{\tau_{1} s^{2}+K \tau_{2} s+K}
$$

Using the relationship of $\tau_{1}, \tau_{2}$ and $\omega_{n}, \mathrm{~K}, \zeta$, the equation can also be shown as:

$$
\frac{\phi(s)}{\phi_{i}(s)}=\frac{s^{2}}{s^{2}+2 \zeta \omega_{n} s+\omega_{n}{ }^{2}}
$$

If a signal is in frequency step version, the input signal will be:

$$
\phi_{i}(t)=\Delta \omega t
$$

The Laplace transform can be shown as:

$$
\phi_{i}(s)=\frac{\Delta \omega}{s^{2}}
$$

Then the function of error signal in frequency domain can be represented as:

$$
\phi(s)=\frac{\omega_{n}{ }^{2}}{s^{2}+2 \zeta \omega_{n} s+\omega_{n}{ }^{2}}
$$

Taking the inverse Laplace transform, error signal $\phi(t)$ can be expressed as:

$$
\begin{array}{cc}
\phi(t)=\frac{\Delta \omega}{\omega_{n}} e^{-\zeta \omega_{n} t \frac{\sinh \left(\omega_{n} \sqrt{\zeta^{2}-1} t\right)}{\sqrt{\zeta^{2}-1}}} & \zeta>1 \\
\phi(t)=\frac{\Delta \omega}{\omega_{n}} e^{-\omega_{n} t} \omega_{n} t & \zeta=1 \\
\phi(t)=\frac{\Delta \omega}{\omega_{n}} e^{-\zeta \omega_{n} t} \frac{\sin \left(\omega_{n} \sqrt{1-\zeta^{2}} t\right)}{\sqrt{1-\zeta^{2}}} & \zeta<1
\end{array}
$$

Using MATLAB, the behavior of equation 3.34 can be shown as: 


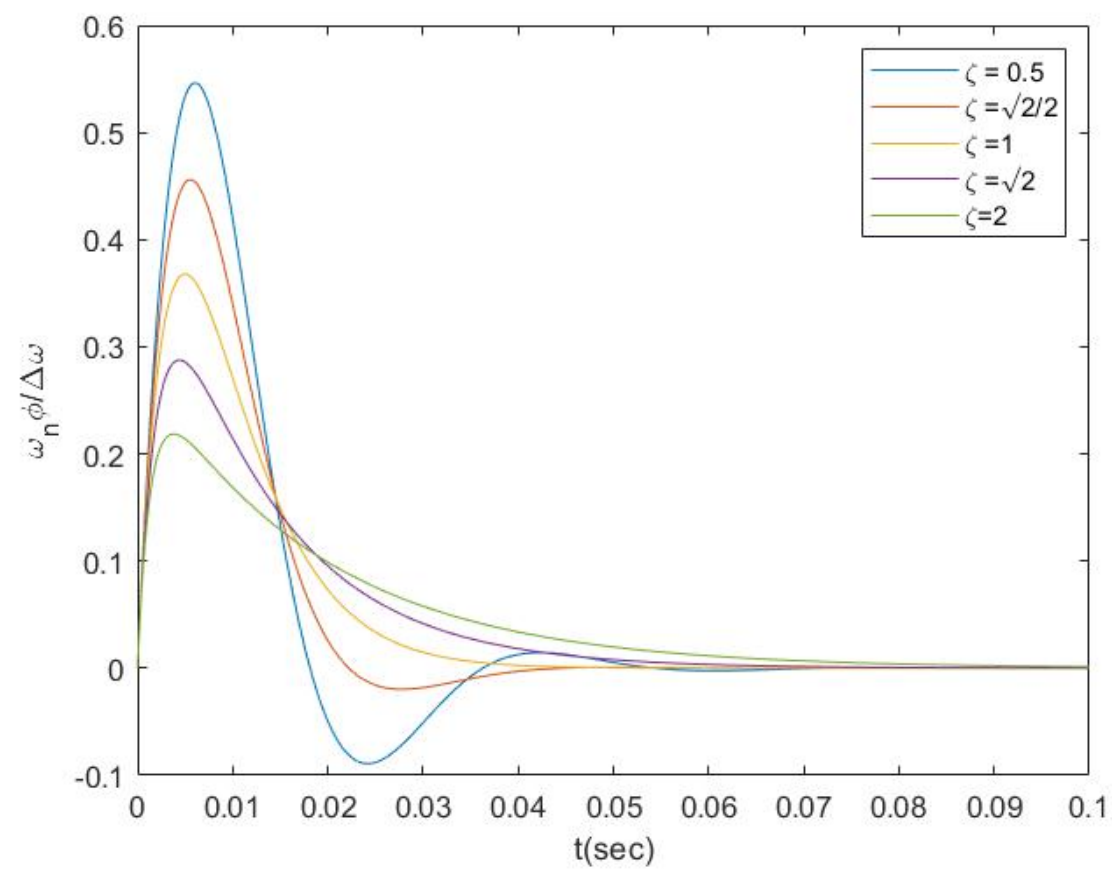

Figure 3-17 Frequency step responses for different values of damping constant $\zeta$

From the Figure 3-17, we can see that as the value of $\zeta$ decreases, the maximum phase error increases, in other words the loop can tolerant bigger phase difference when the input signal comes into the loop. However, smaller $\zeta$ shows less stability for the loop. Considering the above two parameters and the noise, an optimal value of $\zeta$ will be $\frac{\sqrt{2}}{2}$, which can satisfy both the stability and transient requirement. In addition, $\zeta=1$ gives the fastest elimination of the phase error, and $\zeta=\frac{\sqrt{2}}{2}$ is close to 1 .

\subsection{BPSK Costas loop natural acquisition with second-order}

\section{loop filter}


Combing the basic theory of natural acquisition in Chapter 3.1 and the parameters setting for loop filter in Chapter 3.2, it is more useful to build a BPSK Costas loop in Simulink of MATLAB and see how the signal responds.

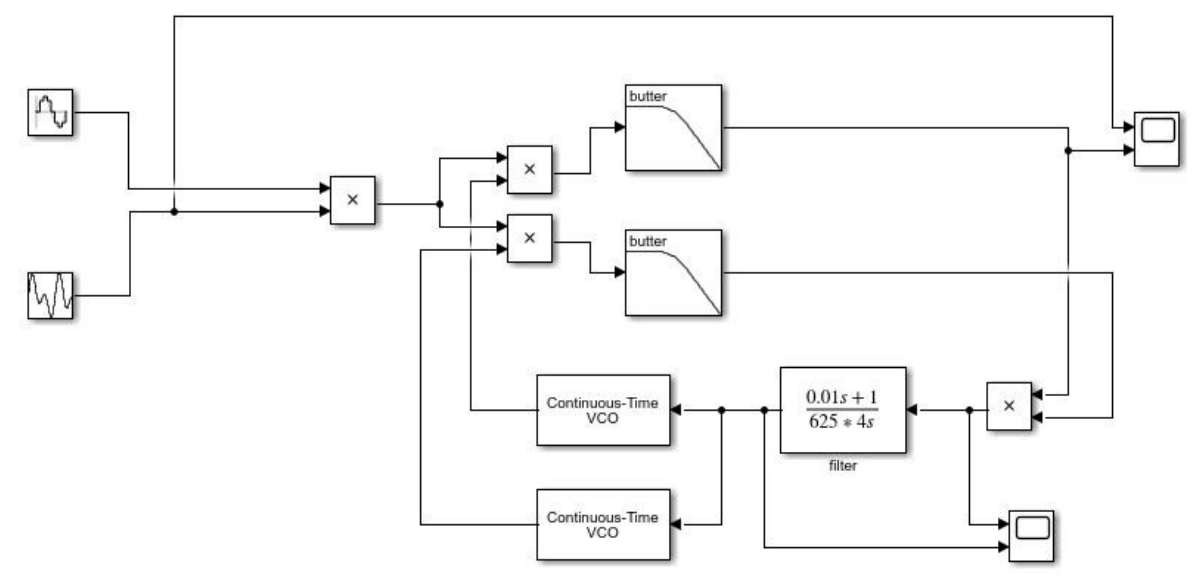

Figure 3-18 Costas loop circuit design in MATLAB Simulink

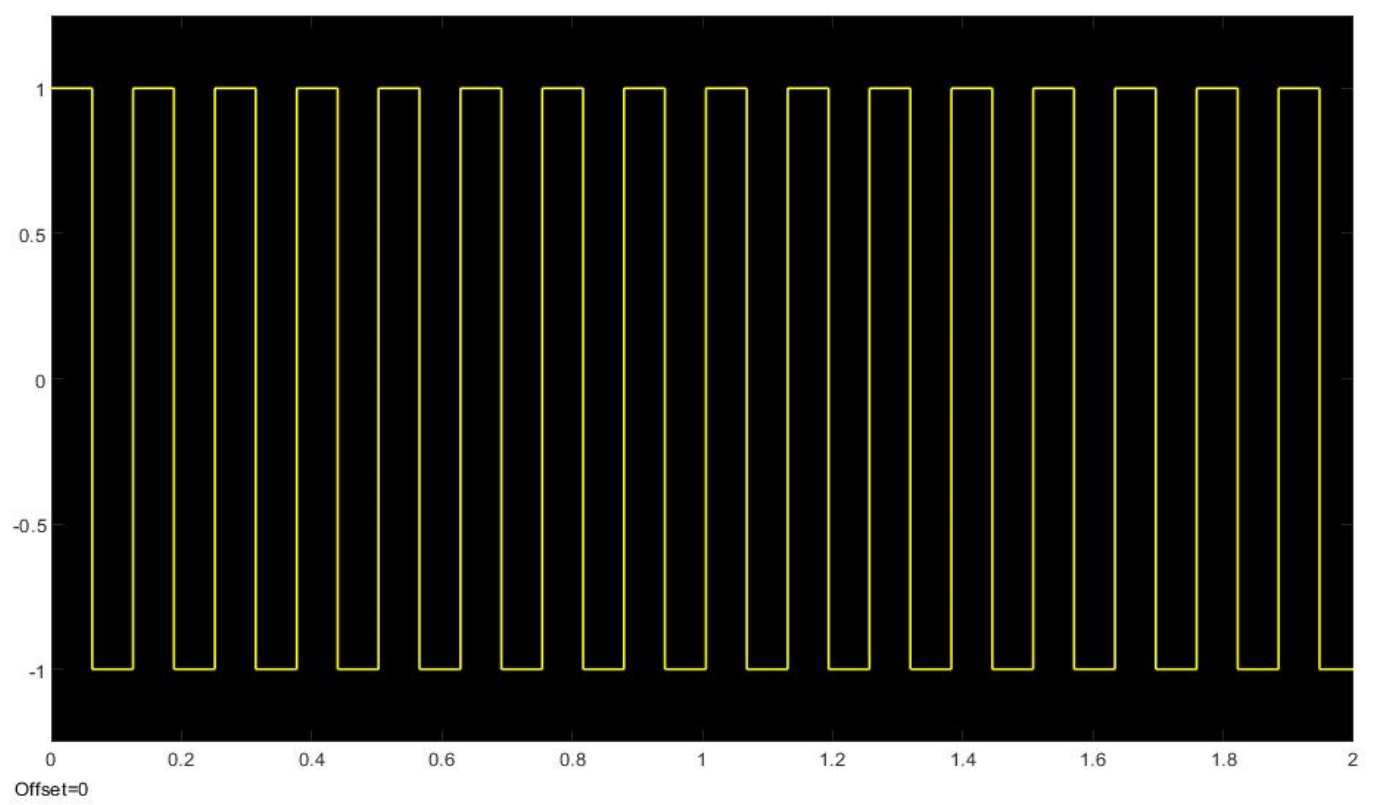

Figure 3-19 The simulation of BPSK data in Simulink (the horizontal axis denotes time with the unit of second, the vertical axis denotes the amplitude, which are applied for all the figures in the Simulink result) 
The data waveform in Figure 3-18 is a BPSK data, with the version of rectangular shape and the frequency of $50 \mathrm{rad} / \mathrm{s}$, the simulation of data waveform is shown in Figure 3-19.

The carrier waveform is $100 \sin (100000 t)$, which illustrates the carrier frequency is $100000 \mathrm{rad} / \mathrm{s}$, and the amplitude of the signal represents the signal sensitivity, being 100 V/rad.

As shown in Figure 3-18, in both I and Q arm, to have a flat frequency response the low pass filter presented in the Simulink is a normalized Butterworth second order LPF.

As for VCO, the original frequency is $99000 \mathrm{rad} / \mathrm{s}$, the amplitude is 2, and the modulation sensitivity $\mathrm{K}_{3}$ is $10000 \mathrm{rad} / \mathrm{s} / \mathrm{V}$.

Similar to the basic Costas loop theory in Chapter 3.1, the input signal will come through both I and Q arm, be multiplied by the in-phase and quadrature-phase local oscillator(LO), pass through the two filters and then multiply together. Consequently, the real $\mathrm{K}_{1}$ becomes:

$K_{1}=\frac{1}{2} K_{1}^{\prime 2}$, where the value of $K_{1}{ }^{\prime}$ is the amplitude of the carrier frequency, in this example, $\mathrm{K}_{1}{ }^{\prime}=100 \mathrm{~V} / \mathrm{rad}$ so $\mathrm{K}_{1}=5000 \mathrm{~V} / \mathrm{rad}$. In other words, the real phase detector sensitivity of the loop $\mathrm{K}_{1}$ is $5000 \mathrm{~V} / \mathrm{rad}$. 


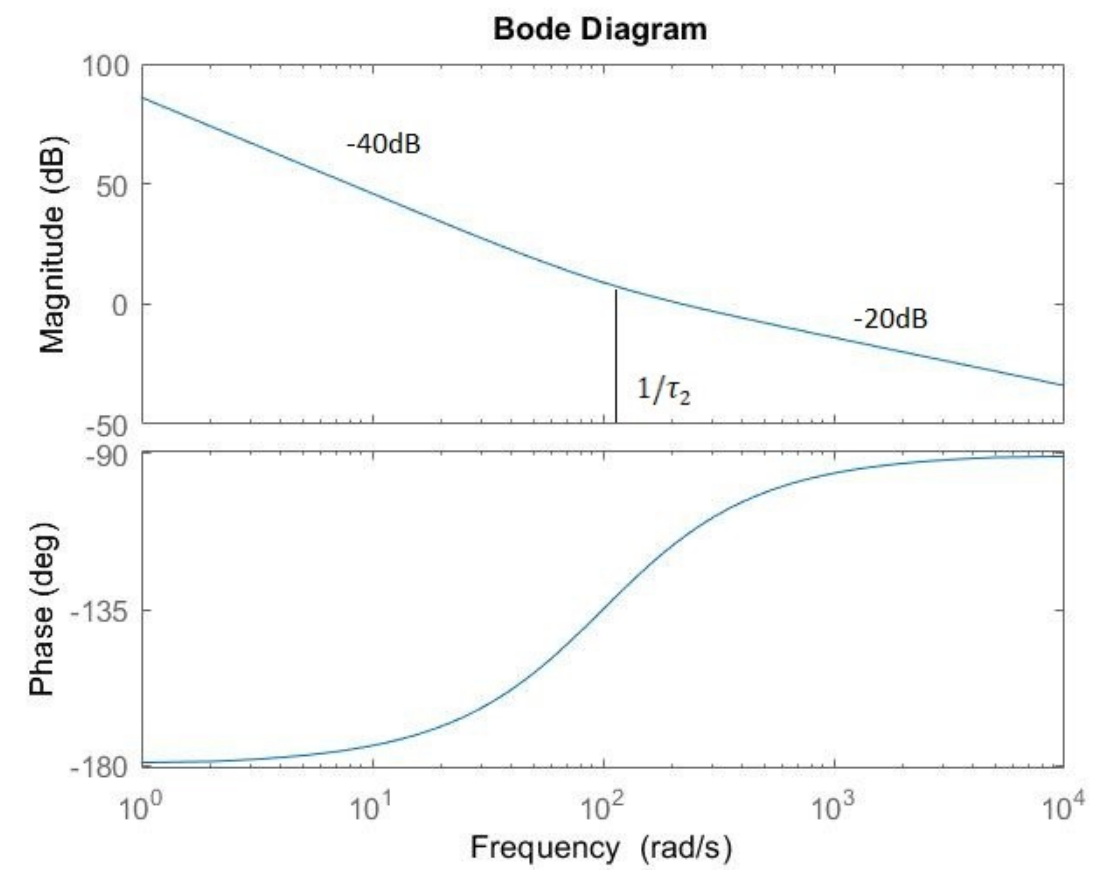

Figure 3-20 Turning point of Bode plot for integrator with phase-lead correction

Recall the Bode plot of the loop filter. Where the magnitude changes from $-40 \mathrm{~dB}$ to $-20 \mathrm{~dB}$, the frequency will be $1 / \tau_{2}$, which can be regarded as the corner frequency $\omega_{c}$. The frequency of BPSK data used is $50 \mathrm{rad} / \mathrm{s}$, and to preserve the data without being distorted by the filter, we can choose the corner frequency to be $100 \mathrm{rad} / \mathrm{s}$, which means $1 / \tau_{2}=100, \tau_{2}=0.01 s$.

Using the relationship between the parameters of the loop filter:

$$
\begin{gathered}
\omega_{n}^{2}=\frac{K}{\tau_{1}} \\
2 \zeta \omega_{n}=\frac{K \tau_{2}}{\tau_{1}}
\end{gathered}
$$

Choose $\zeta=\frac{\sqrt{2}}{2}$, use the value $\mathrm{K}=\mathrm{K}_{1} \mathrm{~K}_{3}=50,000,000 \mathrm{rad} / \mathrm{s}$, and $\tau_{2}=0.01 \mathrm{~s}$, we can calculate that $\tau_{1}=2500 \mathrm{~s}$. 
We still need to pay attention that, as shown in Chapter 3.1, when the signal of I and $\mathrm{Q}$ arm are multiplied in the loop, the frequency before the loop filter becomes $A \sin \left[2\left(\omega_{i}-\omega_{o}\right) t+2\left(\theta_{i}-\theta_{o}\right)\right]$. In other words, the frequency difference between the VCO $\omega_{o}$ and input signal frequency $\omega_{i}$ is twice the frequency difference. As a result, we need to choose the bandwidth of the low pass filter in the two arms to be at least twice as the original frequency difference. In this case, the cut off frequency of the low pass filter is chosen to be $2500 \mathrm{rad} / \mathrm{s}$.

The input before the VCO and the simulation of the demodulated data is shown in Figure 3-21 and Figure 3-22 separately.

From Figure 3-22 can we see when $\mathrm{t}=0.45 \mathrm{~s}$, the frequency achieves synchronization, and the data can be demodulated successfully.

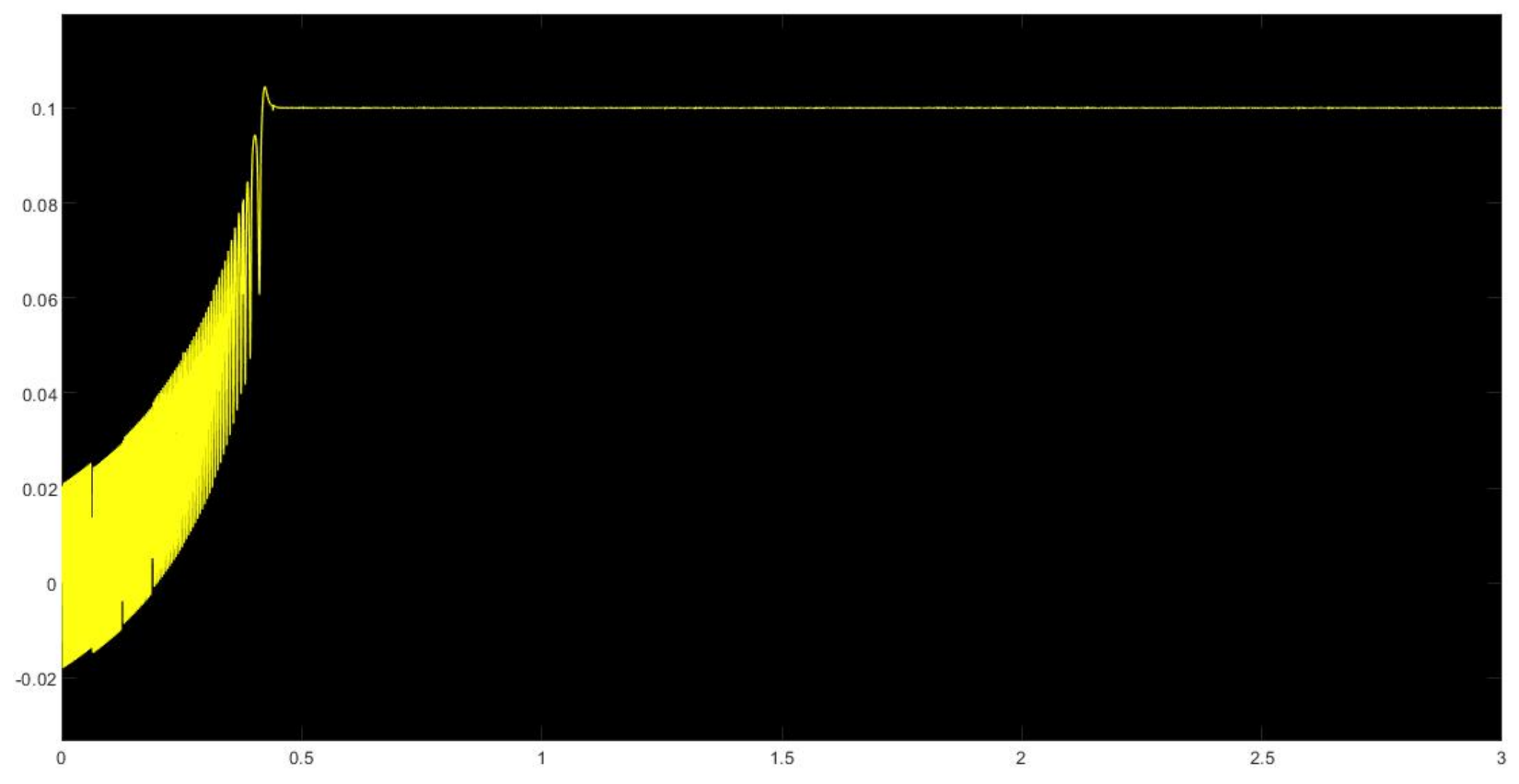



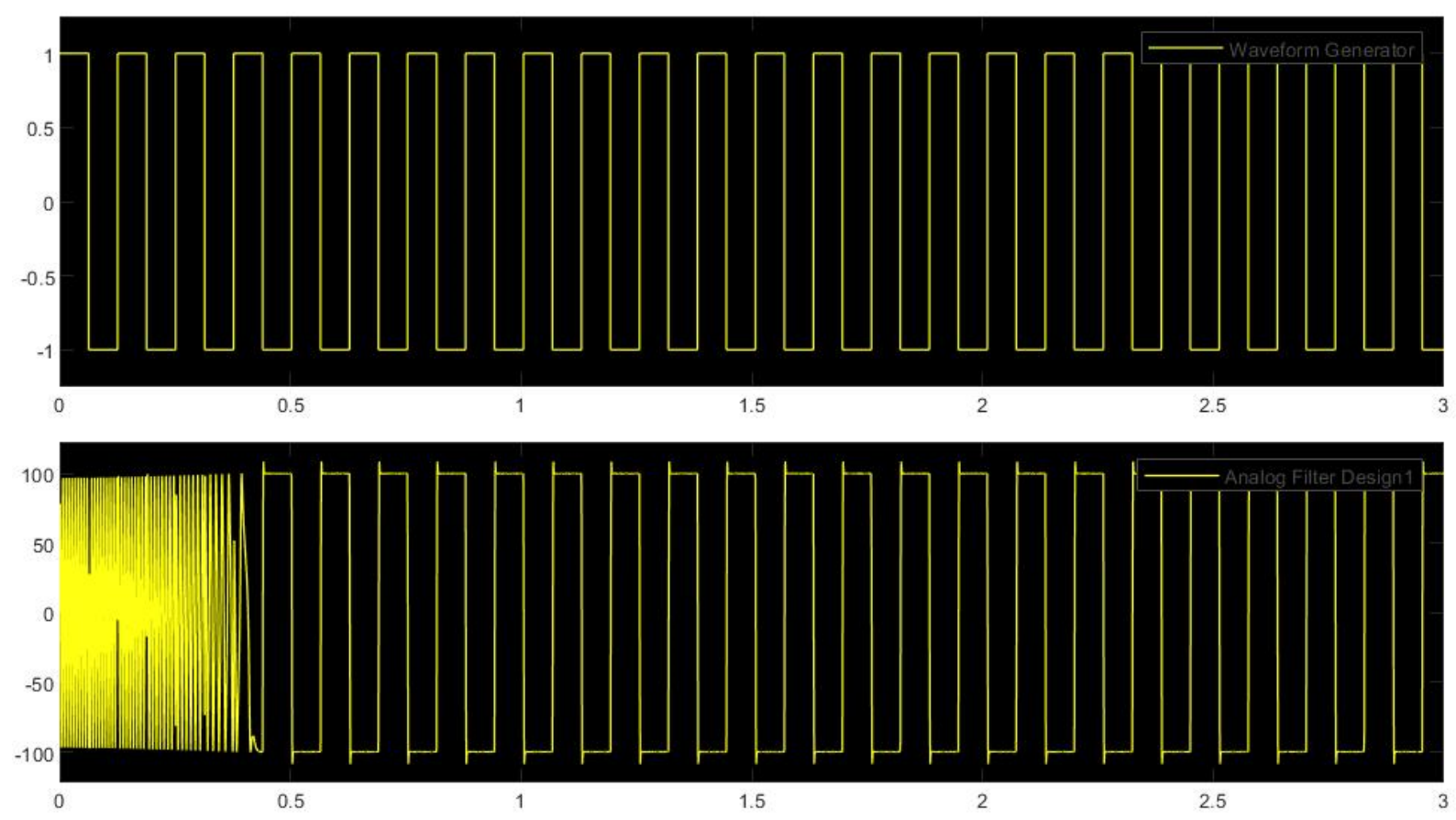

Figure 3-22 Comparison between BPSK and demodulated BPSK signals with Costas loop

\subsection{Fast Methods for Achieving Acquisition}

From the example of Chapter 3.3 can we see that even for the initial frequency offset of just $1000 \mathrm{rad} / \mathrm{s}$, it takes $0.45 \mathrm{~s}$ to achieve frequency synchronization. In the real satellite communication, since high-speed transmission is necessary and fast acquisition is needed, ways of achieving faster acquisition need to be found.

- Change of Parameters

According to the Analytical approximation method in [17], if the initial frequency difference between the input signal and the VCO is $\Omega_{0}$, the acquisition time can be approximately calculated as:

$$
T_{a c q} \approx \frac{\Omega_{0}{ }^{2}}{2 \zeta \omega_{n}{ }^{3}}
$$


A Costas loop is different from the PLL example and the frequency difference arriving at the $\mathrm{VCO}$ is $2 \Omega_{0}$. However, the acquisition time equation is the same. In the equation 3.35, and considering stability, the only parameter to change is the natural frequency $\omega_{n}$ to achieve faster acquisition. We can increase the value of $\omega_{n}$ to decrease the acquisition time. Combining with the equation 3.19, once the parameter $\omega_{n}$ increases, the value of $\tau_{1}$ will decrease.

Compared with the previous value of $\tau_{1}$ of $2500 \mathrm{~s}$, we can give the value of $\tau_{1}$ to be $2000 \mathrm{~s}$, and keep the same value of $\tau_{2}$ and the other parameters in the Simulink

As shown in Figure 3-23, the acquisition has improved compared with Figure 3-22 and the loop can achieve synchronization at $0.3 \mathrm{~s}$, which is smaller than the time when a larger value of $\tau_{1}$ is used.
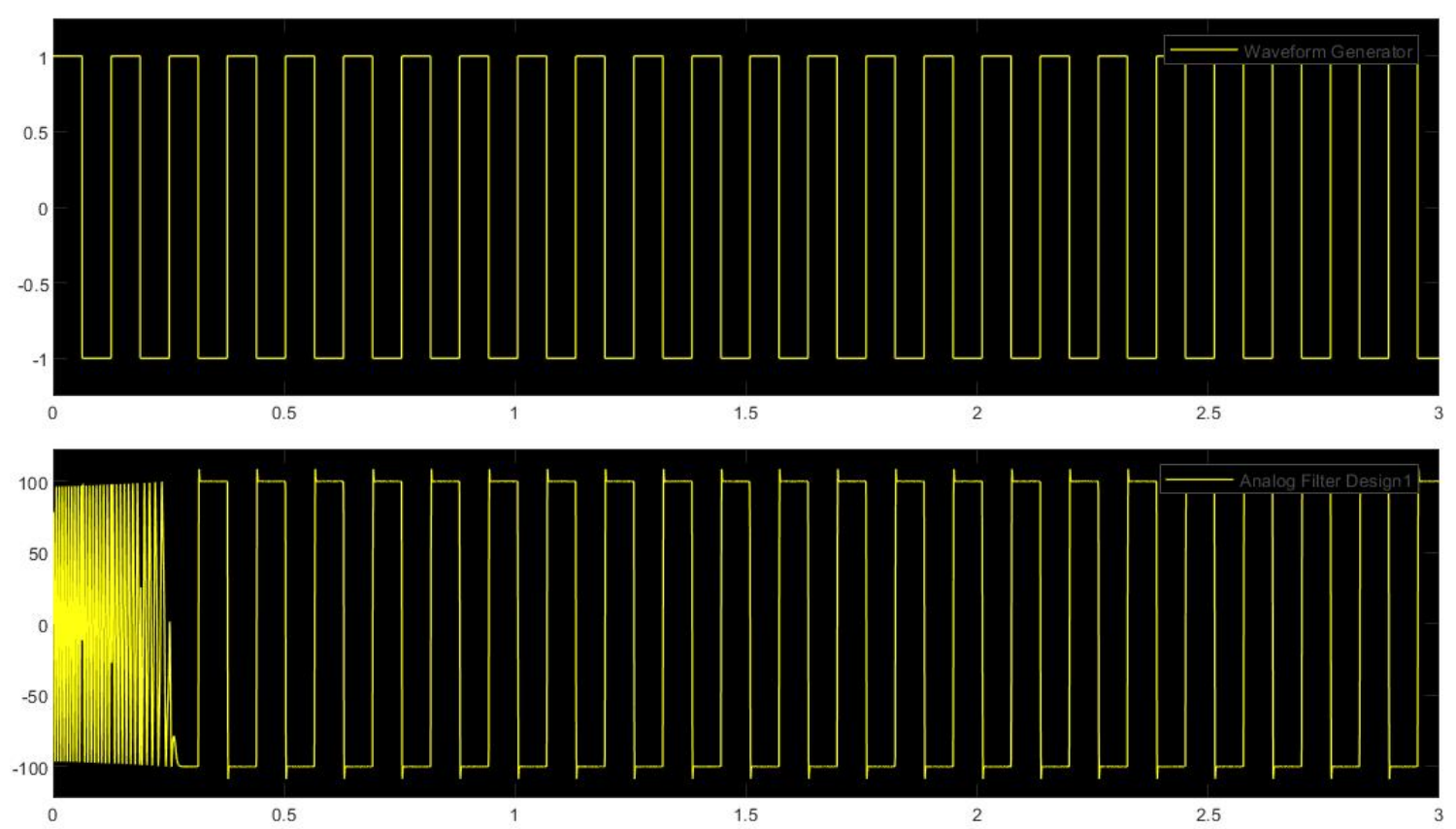

Figure 3-23 Comparison between BPSK and demodulated BPSK by using Costas loop with a smaller value of $\tau_{1}$ 


\section{- Quadri-correlator}

Another way of achieving fast frequency acquisition is to use some auxiliary circuit to aid synchronization such as incorporating a quadri-correlator into the loop. One of the common applications of the quadri-correlator is to use it as frequency discriminator [14]. In addition, the function that the quadri-correlator produces can also give fast acquisition.

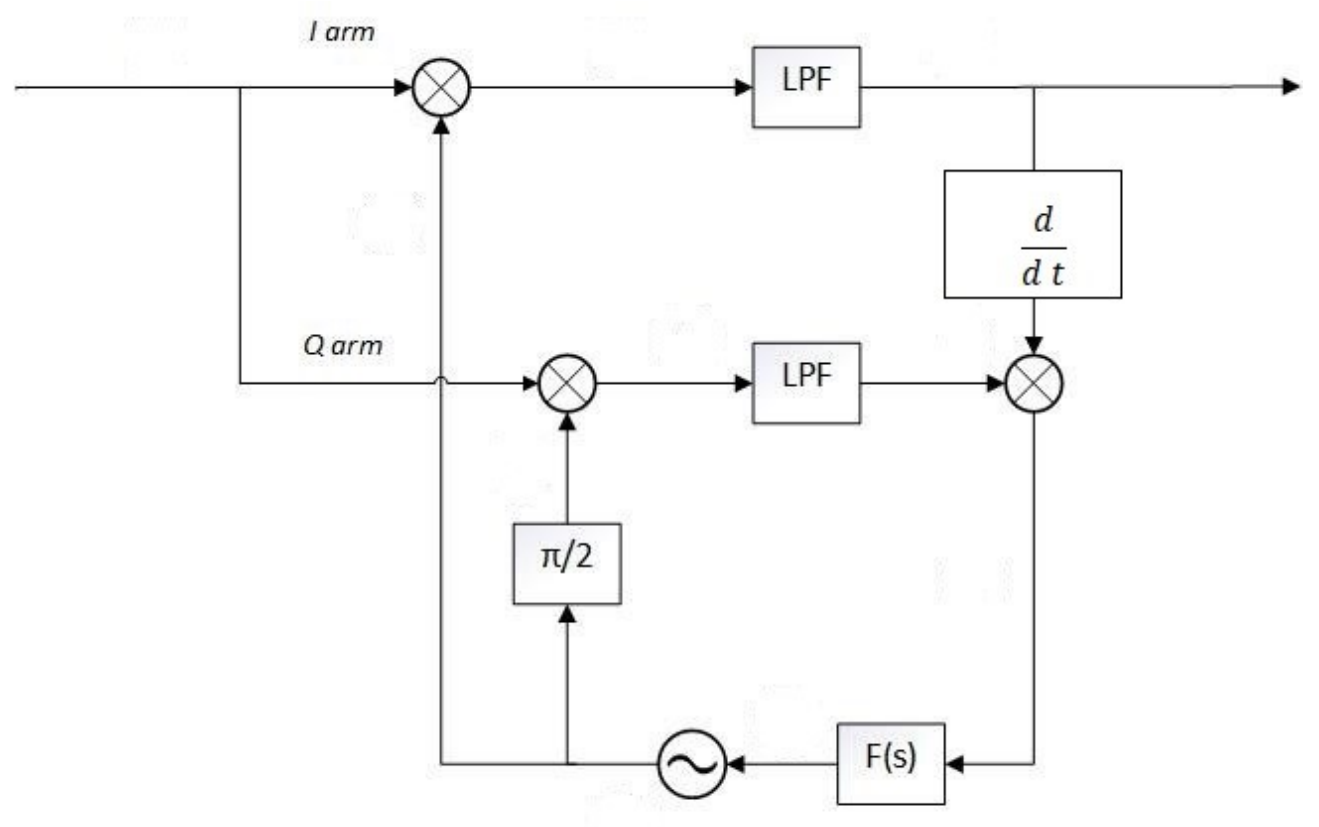

Figure 3-24 The Costas loop with adding a quadri-correlator in I arm

The basic of a quadri-correlator circuit shown in Figure 3-24, and the difference is that there is a derivative function in the I arm after the LPF. The original function before the quadri-correlator in the I arm is $K_{1}^{\prime} \cos \left[\left(\omega_{i}-\omega_{o}\right) t\right] \mathrm{V}$ (ignoring the phase difference). After the derivative function, the equation changes into:

$$
-K_{1}^{\prime}\left(\omega_{i}-\omega_{o}\right) \sin \left[\left(\omega_{i}-\omega_{o}\right) t\right]
$$

Combining this with the function in Q arm after the LPF, $K_{1}^{\prime} \sin \left[\left(\omega_{i}-\omega_{o}\right) t\right]$, the output before the loop filter becomes: 


$$
\frac{-K_{1}^{\prime 2}}{2}\left(\omega_{i}-\omega_{o}\right)\left(1-\cos \left[2\left(\omega_{i}-\omega_{o}\right) t\right]\right)
$$

To guarantee the input before the VCO to be positive, there is a need to add an inversion or negative sign '-' to make the function 3.37 to be greater than 0 . Compared with the function of the BPSK Costas loop without any auxiliary circuit, $\frac{{K_{1}^{\prime}}^{2}}{2} \sin \left(2\left(\omega_{i}-\right.\right.$ $\left.\left.\omega_{o}\right) t\right)$, the function of $\frac{{K_{1}^{\prime}}^{2}}{2}\left(\omega_{i}-\omega_{o}\right)\left(1-\cos \left[2\left(\omega_{i}-\omega_{o}\right) t\right]\right)$ has an obvious larger value at the beginning and sweeps quickly, which helps the VCO respond more quickly to achieve synchronization with the input signal.

Since the coefficient of the function before the loop filter becomes $\frac{{K_{1}^{\prime}}^{2}}{2}\left(\omega_{i}-\omega_{o}\right)$, the real synchronization bandwidth $K$ of the loop also varies as $\left(\omega_{i}-\omega_{o}\right)$ times as the previous one, which gives:

$$
K=\frac{K_{1}^{\prime 2} K_{3}}{2}\left(\omega_{i}-\omega_{o}\right)
$$

Back with the same example of the BPSK Costas loop, using the same parameters, the value of $\mathrm{K}$ will become $50,000,000 \times 1000=5 \times 10^{10} \mathrm{rad} / \mathrm{s}, \tau_{1}$ also increases into $2,500,000 \mathrm{~s}$.

Consequently, the loop filter function will be:

$$
F(s)=\frac{0.01 s+1}{2500000 s}
$$

The circuit design in Simulink and the result of demodulated data with the help of quadri-correlator are shown in Figure 3-25 and 3-26 separately: 


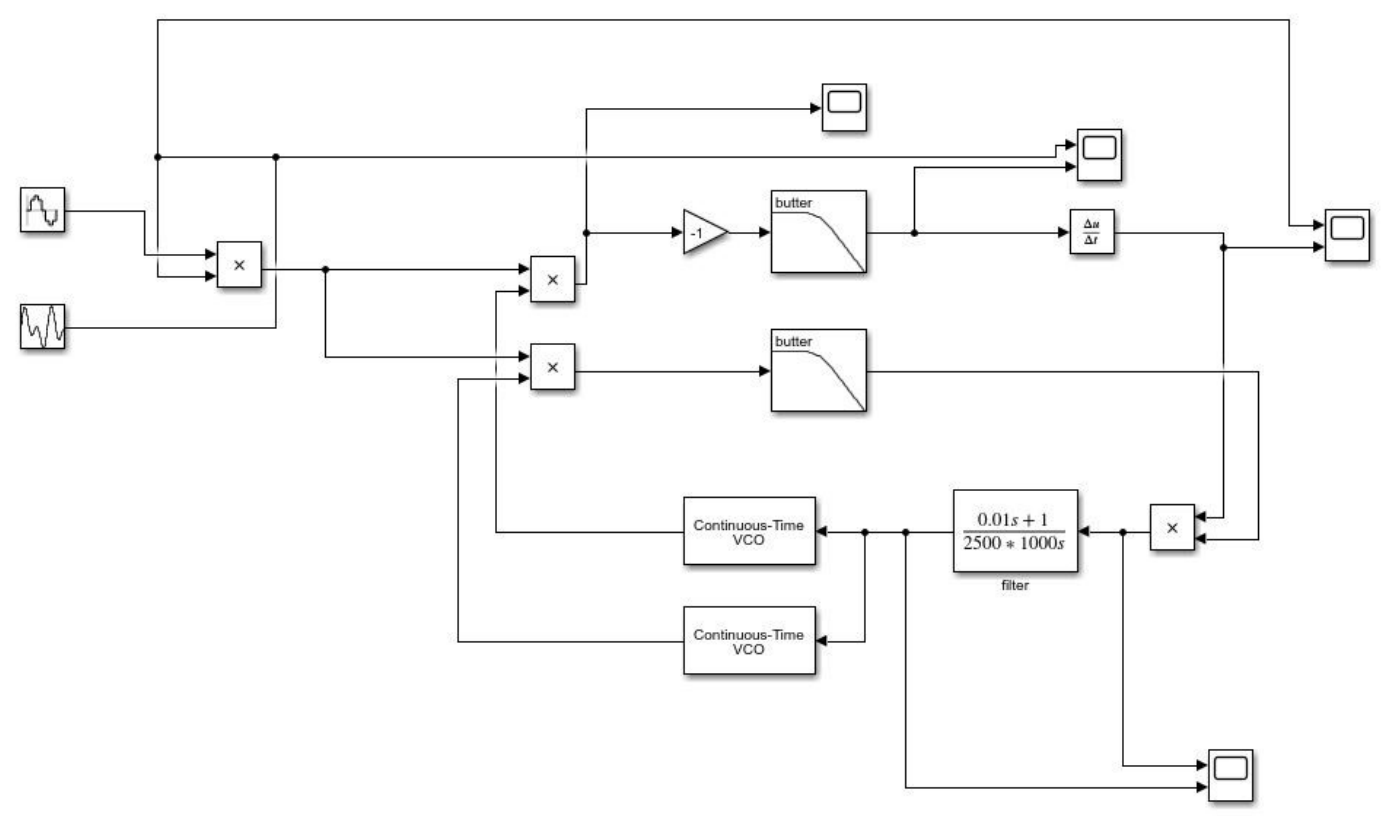

Figure 3-25 A Costas loop with a quadri-correlator circuit design in MATLAB Simulink
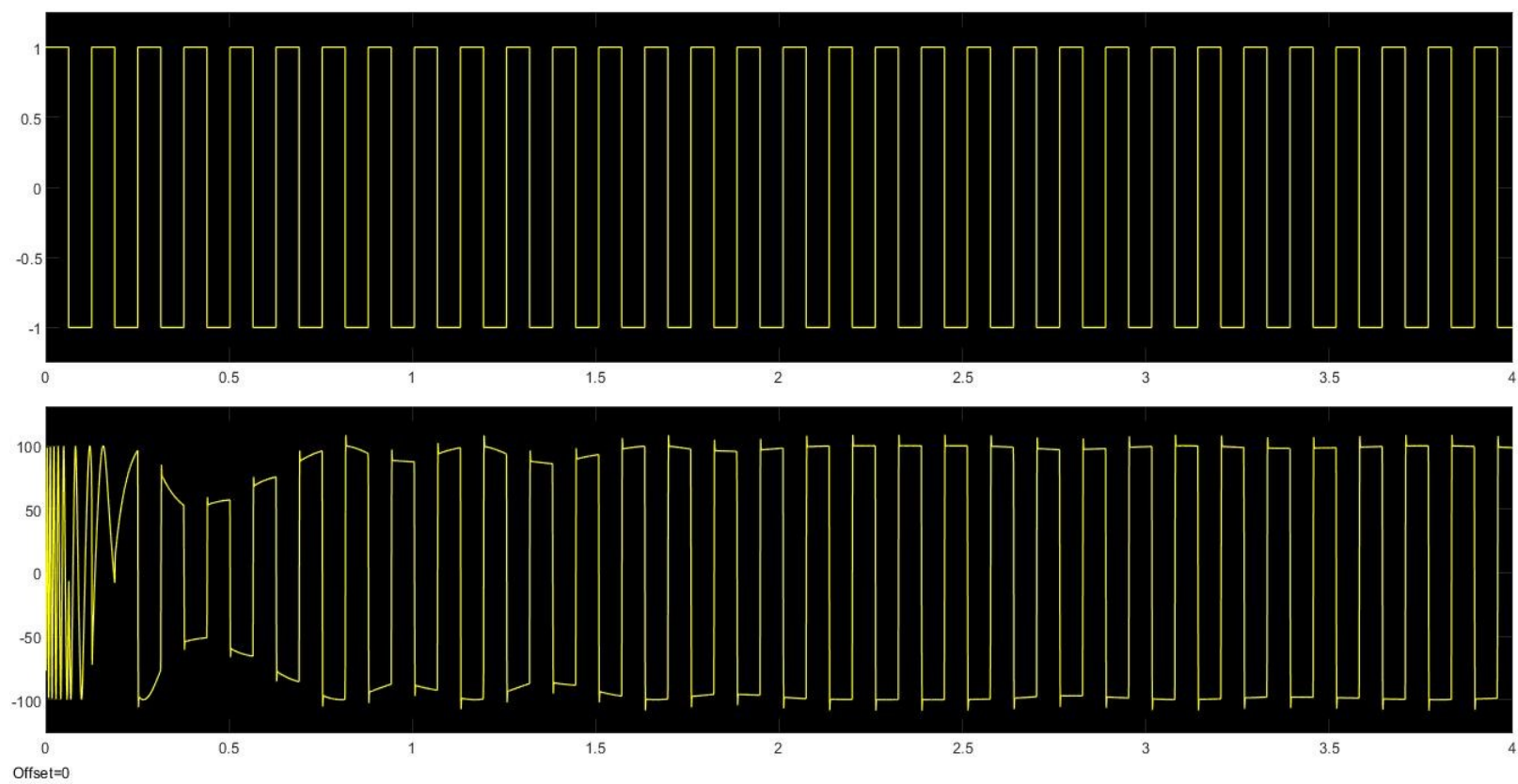

Figure 3-26 Comparison between BPSK and demodulated BPSK by using a Costas loop with a quadri-correlator 
Compared with Figure 3-22, the frequency acquisition of a common Costas loop, using the same parameters, Figure 3-26 shows an obvious shorter time of achieving synchronization at around $0.25 \mathrm{~s}$. Nevertheless, the quadri-correlator added in the circuit also brings the drawback that when the data first achieves synchronization, there is some distortion for the BPSK waveform as shown in the figure. However, we can still improve the demodulation by further optimizing the loop.

\section{- Optimization in quadri-correlator}

As shown in previous simulation, the common Costas loop can demodulate the data with better integrity when the loop is synchronized while the addition of quadri-correlator can increase the speed of acquisition. An efficient optimizing way can accommodate a combination of the two loops.

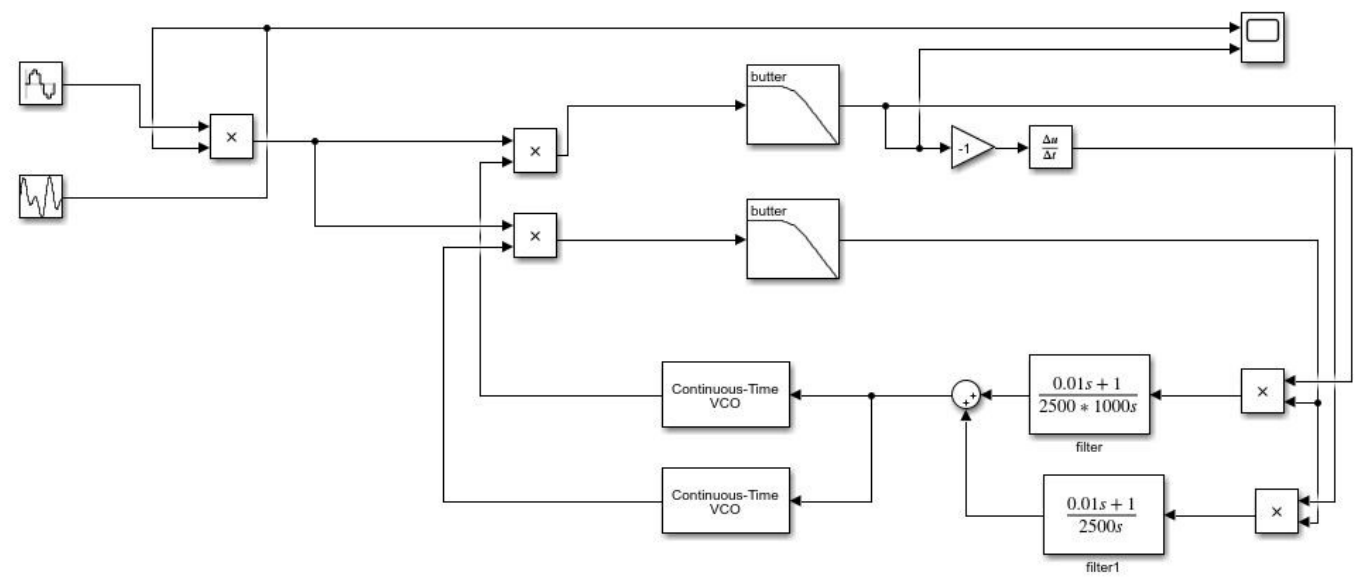

Figure 3-27 The circuit construction of combing a Costas loop and a Costas loop with a quadricorrelator in Simulink 
As shown in Figure 3-27, in the I arm of the Costas loop, there are two branches where the signals enter simultaneous, one of which is the traditional Costas loop and the other is the Costas loop with the quadri-correlator. The signals in the two branches both multiply with the signal from Q arm and go in into their own filters. Finally, the two signals add together and modulate the VCO.
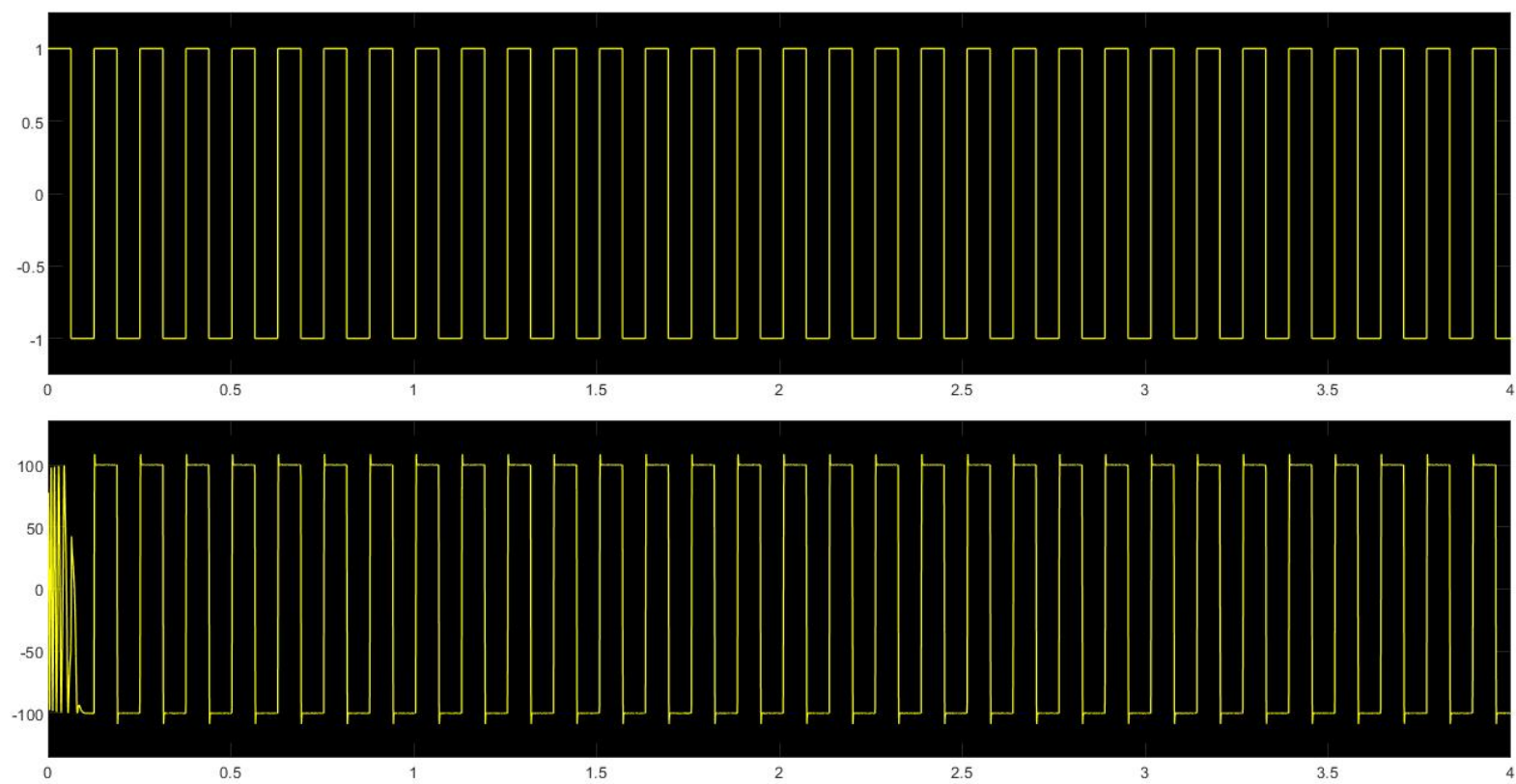

Figure 3-28 Comparison between BPSK and BPSK demodulated by the optimized a Costas loop with a quadri-correlator

From Figure 3-28, we can see that the combination of the two ways of achieving synchronization can improve the frequency acquisition to a great extent. Not only the integrity of BPSK signals can be kept with less distortion, the synchronous time can be reduced at $0.1 \mathrm{~s}$.

\subsection{Summary}


Extending the theory from PLL to Costas loop, the constructed BPSK Costas loops can achieve synchronization and demodulate the signals from the carrier. Especially for the new design, the combination of traditional Costas loop and the Costas loop with quadricorrelator can, not only decrease the acquisition time by a huge extent, but also present less distortion after synchronization, which satisfies the requirement of $5 \mathrm{G}$ concept. Apart from BPSK signals, another commonly used phase modulation is quaternary phase-shift keying (QPSK); likewise, if we want to demodulate QPSK signals from the carrier, the construction of a QPSK Costas loop is a requisite. 


\section{Chapter 4}

\section{QPSK Costas Loop and Natural Acquisition}

QPSK has four possible phases of the carrier that convey the transmitted information. Different from BPSK presenting only 0 or 1, QPSK can transmit two bits per symbol, so the combination of the QPSK transmitted message per symbol can be 00,01 , 10 or 11 [19]. Figure 4-1 shows the signal space diagram for QPSK.

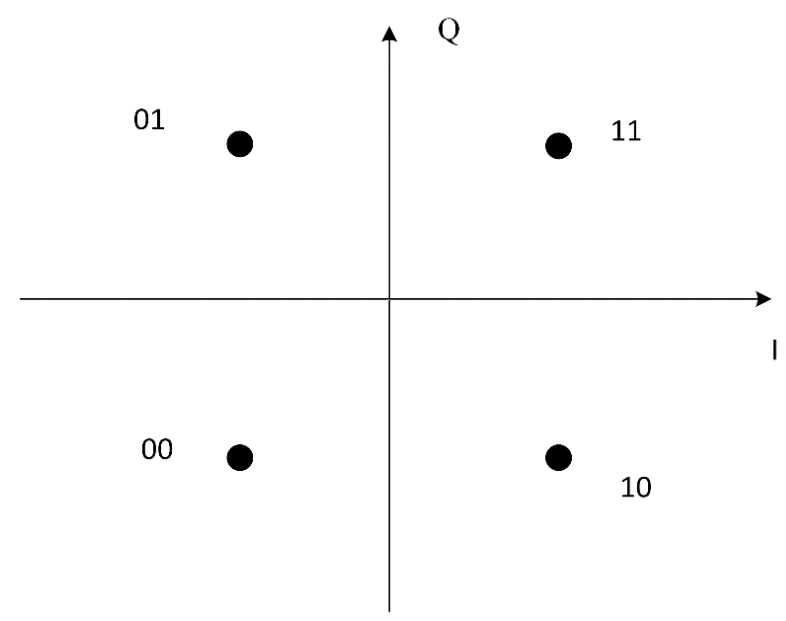

Figure 4-1 Signal space diagram for QPSK

Again, as for BPSK, in the simulations, we use -1 to represent the signal 0.

\subsection{Basic theory for QPSK Costas loop}




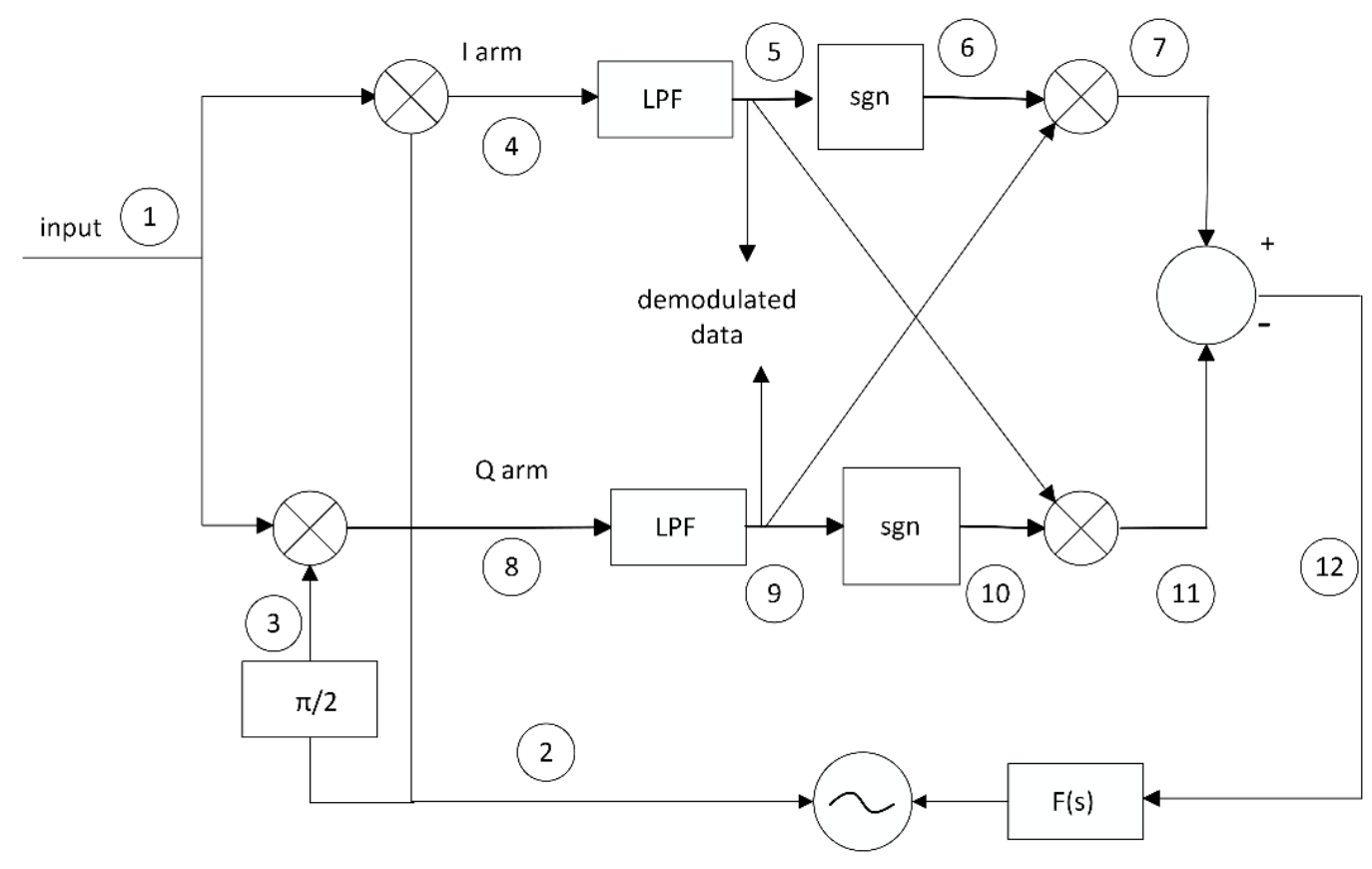

Figure 4-2 The diagram of a QPSK Costas loop

Since there are two bits per symbol in QPSK modulation, the input (1) shown in the Figure 4-2 will be $m(t) \sin \left(\omega_{i} t+\theta_{i}\right)+n(t) \cos \left(\omega_{i} t+\theta_{i}\right)$, where $\mathrm{m}(\mathrm{t})$ and $\mathrm{n}(\mathrm{t})$ are two BPSK data sets, $\omega_{i}$ is the carrier frequency and $\theta_{i}$ is the initial phase of the carrier. The signal in (2) represents the initial output of the $\mathrm{VCO}$, and is $2 \sin \left(\omega_{o} t+\theta_{o}\right)$, where $\omega_{o}$ and $\theta_{o}$ denote the initial frequency and phase of the VCO separately.

In the I arm, after the input and the signal of the VCO are multiplied, the signal shown at (4) becomes: $2\left[m(t) \sin \left(\omega_{i} t+\theta_{i}\right)+n(t) \cos \left(\omega_{i} t+\theta_{i}\right)\right] \sin \left(\omega_{o} t+\theta_{o}\right)$. Using the sinusoidal law, this equation is equal to:

$$
\begin{aligned}
& m(t) \cos \left[\left(\omega_{i}-\omega_{0}\right) t+\left(\theta_{i}-\theta_{0}\right)\right]-m(t) \cos \left[\left(\omega_{i}+\omega_{0}\right) t+\left(\theta_{i}+\theta_{0}\right)\right]+ \\
& n(t) \sin \left[\left(\omega_{i}+\omega_{0}\right) t+\left(\theta_{i}+\theta_{0}\right)\right]-n(t) \sin \left[\left(\omega_{i}-\omega_{0}\right) t+\left(\theta_{i}-\theta_{0}\right)\right]
\end{aligned}
$$

The LPF will filter out the higher frequency of the signal, so the signal left at (5) will be: 


$$
m(t) \cos \left[\left(\omega_{i}-\omega_{0}\right) t+\left(\theta_{i}-\theta_{0}\right)\right]-n(t) \sin \left[\left(\omega_{i}-\omega_{0}\right) t+\left(\theta_{i}-\theta_{0}\right)\right]
$$

After the hard-limiting sign function, the function in (6) then changes to:

$$
\operatorname{sgn}\left\{m(t) \cos \left[\left(\omega_{i}-\omega_{0}\right) t+\left(\theta_{i}-\theta_{0}\right)\right]-n(t) \sin \left[\left(\omega_{i}-\omega_{0}\right) t+\left(\theta_{i}-\theta_{0}\right)\right]\right\}
$$

The input signal to the $\mathrm{Q}$ arm is the same as that at the I arm. However, the LO is shifted by 90 degrees.

The output of LO at (3) then becomes $2 \cos \left(\omega_{o} t+\theta_{o}\right)$, which is multiplied by the input, to give:

$$
2\left[m(t) \sin \left(\omega_{i} t+\theta_{i}\right)+n(t) \cos \left(\omega_{i} t+\theta_{i}\right)\right] \cos \left(\omega_{o} t+\theta_{o}\right) .
$$

Using the sinusoidal law, after the LPF in the Q arm, the signals in (9) becomes:

$$
m(t) \sin \left[\left(\omega_{i}-\omega_{0}\right) t+\left(\theta_{i}-\theta_{0}\right)\right]+n(t) \cos \left[\left(\omega_{i}-\omega_{0}\right) t+\left(\theta_{i}-\theta_{0}\right)\right]
$$

Then in 10 the hard-limiting operation function will make the function into:

$$
\operatorname{sgn}\left\{m(t) \sin \left[\left(\omega_{i}-\omega_{0}\right) t+\left(\theta_{i}-\theta_{0}\right)\right]+n(t) \cos \left[\left(\omega_{i}-\omega_{0}\right) t+\left(\theta_{i}-\theta_{0}\right)\right]\right\}
$$

Following the sign function, there is a crossed multiplication between the I and Q arm. Finally, the function before the loop filter shown in (12) becomes:

$$
\begin{aligned}
& \operatorname{sgn}[m(t) \cos (\Delta \omega t+\Delta \theta)-n(t) \sin (\Delta \omega t+\Delta \theta)] m(t)[\sin (\Delta \omega t+\Delta \theta) \\
& +n(t) \cos (\Delta \omega t+\Delta \theta)]-\operatorname{sgn}[m(t) \sin (\Delta \omega t+\Delta \theta)+n(t) \cos (\Delta \omega t \\
& +\Delta \theta)][m(t) \cos (\Delta \omega t+\Delta \theta)-n(t) \sin (\Delta \omega t+\Delta \theta)] \\
& \left(\text { where } \Delta \omega=\omega_{i}-\omega_{0} \text { and } \Delta \theta=\theta_{i}-\theta_{0}\right)
\end{aligned}
$$

A further analysis needs to be made for the equation 4.5. Let $\Delta \omega t+\Delta \theta=\theta(t)$, so the expression of the 4.5 depends on the time t. Equation 4-5 can be simplified over four separate regions of $\theta(t)$. 
When: $-\frac{\pi}{4}<\theta(t)<\frac{\pi}{4}, \cos \theta(t)>\sin \theta(t)$, then in the expression 4.5 :

$$
\begin{aligned}
& \operatorname{sgn}[m(t) \cos (\Delta \omega t+\Delta \theta)-n(t) \sin (\Delta \omega t+\Delta \theta)] \approx m(t) \\
& \operatorname{sgn}[m(t) \sin (\Delta \omega t+\Delta \theta)+n(t) \cos (\Delta \omega t+\Delta \theta)] \approx n(t)
\end{aligned}
$$

Since $m(t)$ and $n(t)$ both represent BPSK signals, we can choose $|m(t)|=|n(t)|=$ 1. The function 4.5 can then be approximately equal as $2 \sin \theta(t)$.

Using the same technique, when $\frac{\pi}{4}<\theta(t)<\frac{3 \pi}{4}, \sin \theta(t)>\cos \theta(t)$. Then the expression 4.5 can be written:

$$
\begin{aligned}
& \operatorname{sgn}[m(t) \cos (\Delta \omega t+\Delta \theta)-n(t) \sin (\Delta \omega t+\Delta \theta)] \approx-n(t) \\
& \operatorname{sgn}[m(t) \sin (\Delta \omega t+\Delta \theta)+n(t) \cos (\Delta \omega t+\Delta \theta)] \approx m(t)
\end{aligned}
$$

Then the equation 4-5 can be approximated as $-2 \cos \theta(t)$.

Finally, the condition of $\theta(t)$ is in four cases, and the function of $\mathrm{u}_{\mathrm{d}}(\mathrm{t})(4.5)$ can be summarized as:

$$
\mathrm{u}_{\mathrm{d}}(\mathrm{t}) \approx\left\{\begin{array}{cc}
2 \sin \theta(t) & -\frac{\pi}{4}<\theta(t)<\frac{\pi}{4} \\
-2 \cos \theta(t) & \frac{\pi}{4}<\theta(t)<\frac{3 \pi}{4} \\
-2 \sin \theta(t) & \frac{3 \pi}{4}<\theta(t)<\frac{5 \pi}{4} \\
2 \cos \theta(t) & \frac{5 \pi}{4}<\theta(t)<\frac{7 \pi}{4}
\end{array}\right.
$$

The piecewise function 4.6 is shown in Figure 4-3: 


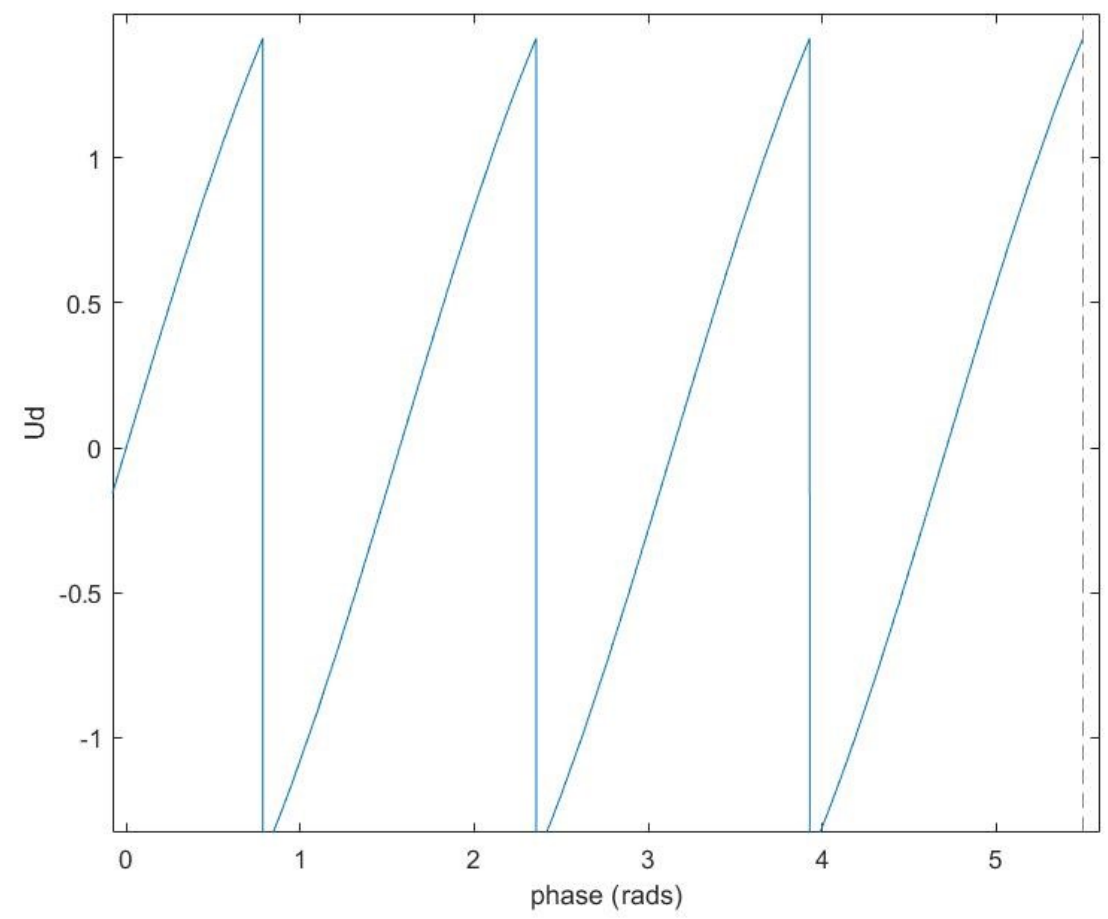

Figure 4-3 The piecewise function of output of the phase detector for a QPSK Costas loop

When the signal achieves synchronization, in (5) of the I arm the equation will become $m(t) \cos (0)=m(t)$, and in (9) of the $\mathrm{Q}$ arm the equation changes into $n(t) \cos (0)=n(t)$.

The data can be demodulated in the two arms to recompose the QPSK data.

\subsection{Phase and frequency acquisition}

To observe a detailed trend of achieving synchronization, it is better to do the simulation in Simulink to see the process of acquisition. The Simulink is shown in the Figure 4-4: 


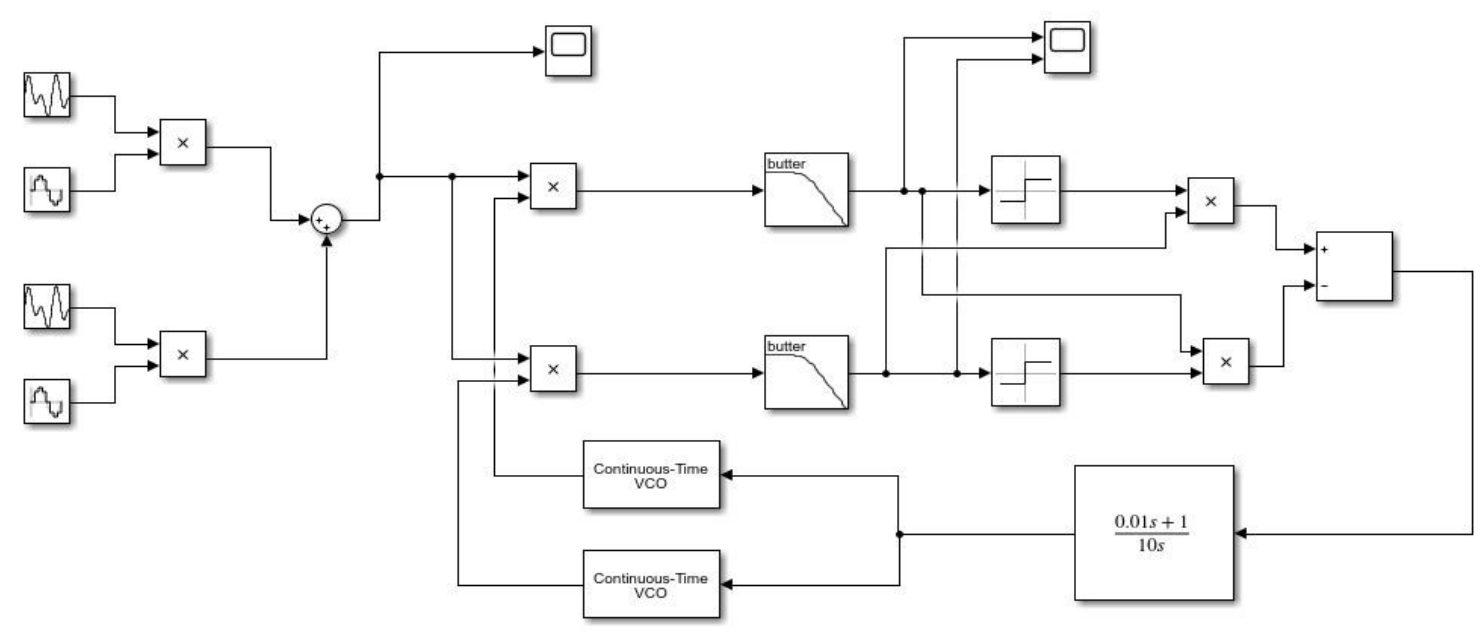

Figure 4-4 Simulink for a QPSK Costas loop in MATLAB
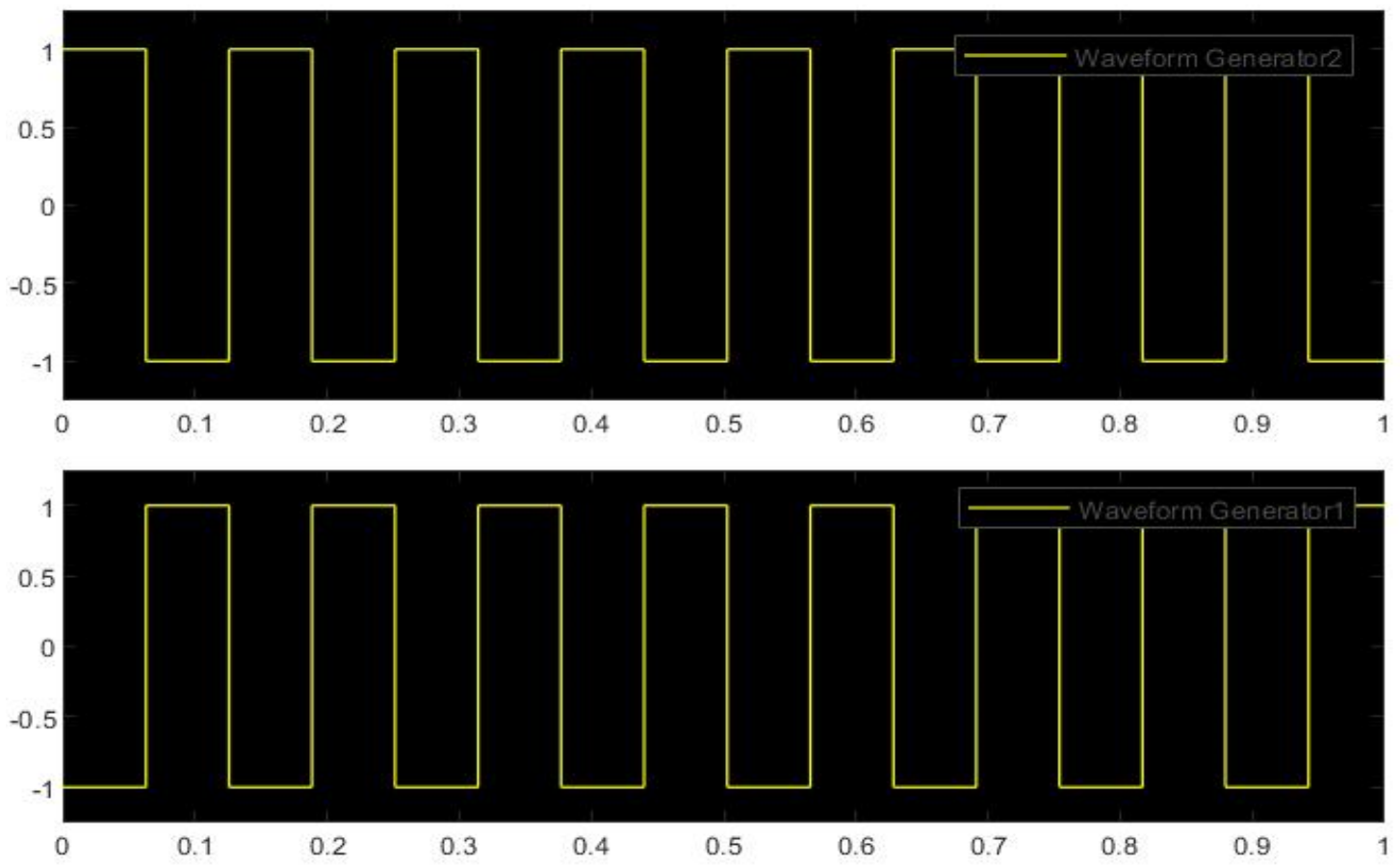

Figure 4-5 Data for $m(t)$ and $n(t)$ 
The transmitted signals here are still rectangular waveforms with the frequency 50 $\mathrm{rad} / \mathrm{s}$. To distinguish the two signals of $m(t)$ and $n(t)$, there is an initial phase difference between them as shown in Figure 4-5.

As shown in Figure 4-5, the upper waveform represents BPSK data $m(t)$ and the lower one shows the BPSK data $\mathrm{n}(\mathrm{t})$. The input signal is $K_{1}\left\{m(t) \sin \left(\omega_{i} t+\theta_{i}\right)+\right.$ $\left.n(t) \cos \left(\omega_{i} t+\theta_{i}\right)\right\}$. In the simulation, the frequency of the carrier waveform is chosen to be $100,000 \mathrm{rad} / \mathrm{s}$, which means $\omega_{i}=100,000 \mathrm{rad} / \mathrm{s}$. The initial phase used is $\frac{\pi}{6}$, and $\mathrm{K}_{1}$ is chosen as $100 \mathrm{~V} / \mathrm{rad}$. The input signal can be shown in Figure 4-6.

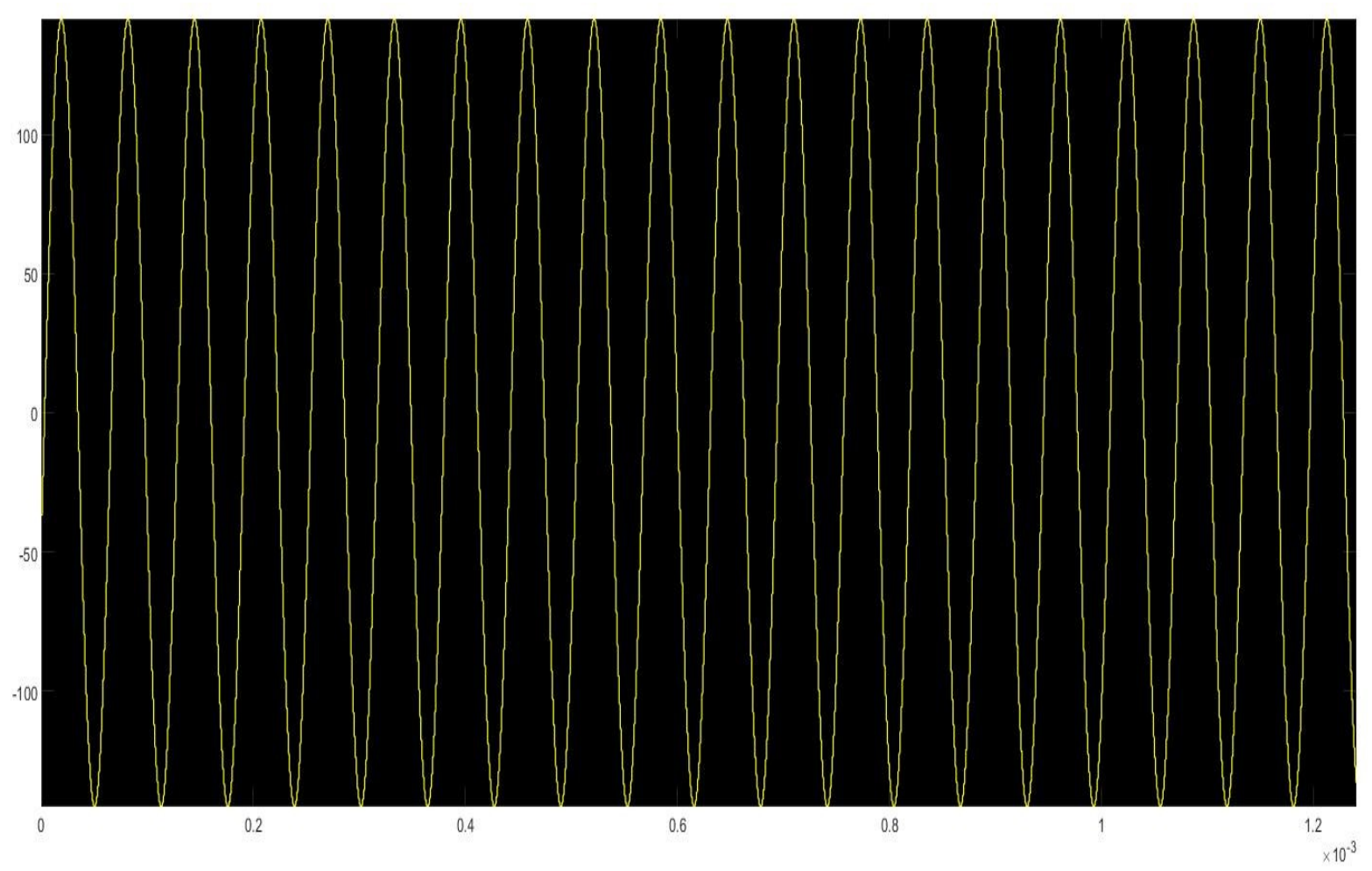

Figure 4-6 The input of the QPSK

The low pass filter used in both I and Q arm in Figure 4-3 is again a normalized Butterworth low pass filter. The sensitivity of the VCO shown in the figure is $\mathrm{K}_{3}=1000$ $\mathrm{rad} / \mathrm{s} / \mathrm{V}$, and the amplitude of the output is $2 \mathrm{~V}$. 
Recall the analysis in Chapter 3.2. Considering the stability and transient response of the loop filter, it is better to choose the damping constant $\zeta=\frac{\sqrt{2}}{2}$ to optimize the loop. Again, equation 3.19 and 3.20 still work for the parameters of the loop filter:

$$
\begin{gathered}
\omega_{n}{ }^{2}=\frac{K}{\tau_{1}} \\
2 \zeta \omega_{n}=\frac{K \tau_{2}}{\tau_{1}}
\end{gathered}
$$

Unlike the BPSK Costas loop with the multiplication of the I and Q arms, in the QPSK Costas loop, there is a difference value function between the two arms before the loop filter in QPSK. Consequently, the value of the phase detector sensitivity $\mathrm{K}_{1}{ }^{\prime}=2 \mathrm{~K}_{1}$. The synchronization bandwidth $\mathrm{K}$ should be calculated as:

$$
K=2 K_{1} K_{3}
$$

In the above case the value of $\mathrm{K}$ will be $2 \times 100 \times 1000=200,000 \mathrm{rad} / \mathrm{s}$.

Using the value $\zeta=\frac{\sqrt{2}}{2}$, and $\tau_{2}=0.01 \mathrm{~s}$, the value of $\tau_{1}$ can be computed as $10 \mathrm{~s}$, so the function of the loop filter will be:

$$
F(s)=\frac{0.01 s+1}{10 s}
$$

\section{- Phase acquisition}

When the frequency of the VCO is already the same as the carrier frequency, the process of the synchronization will only be the phase acquisition. Figure 4-7 shows the phase acquisition process: 

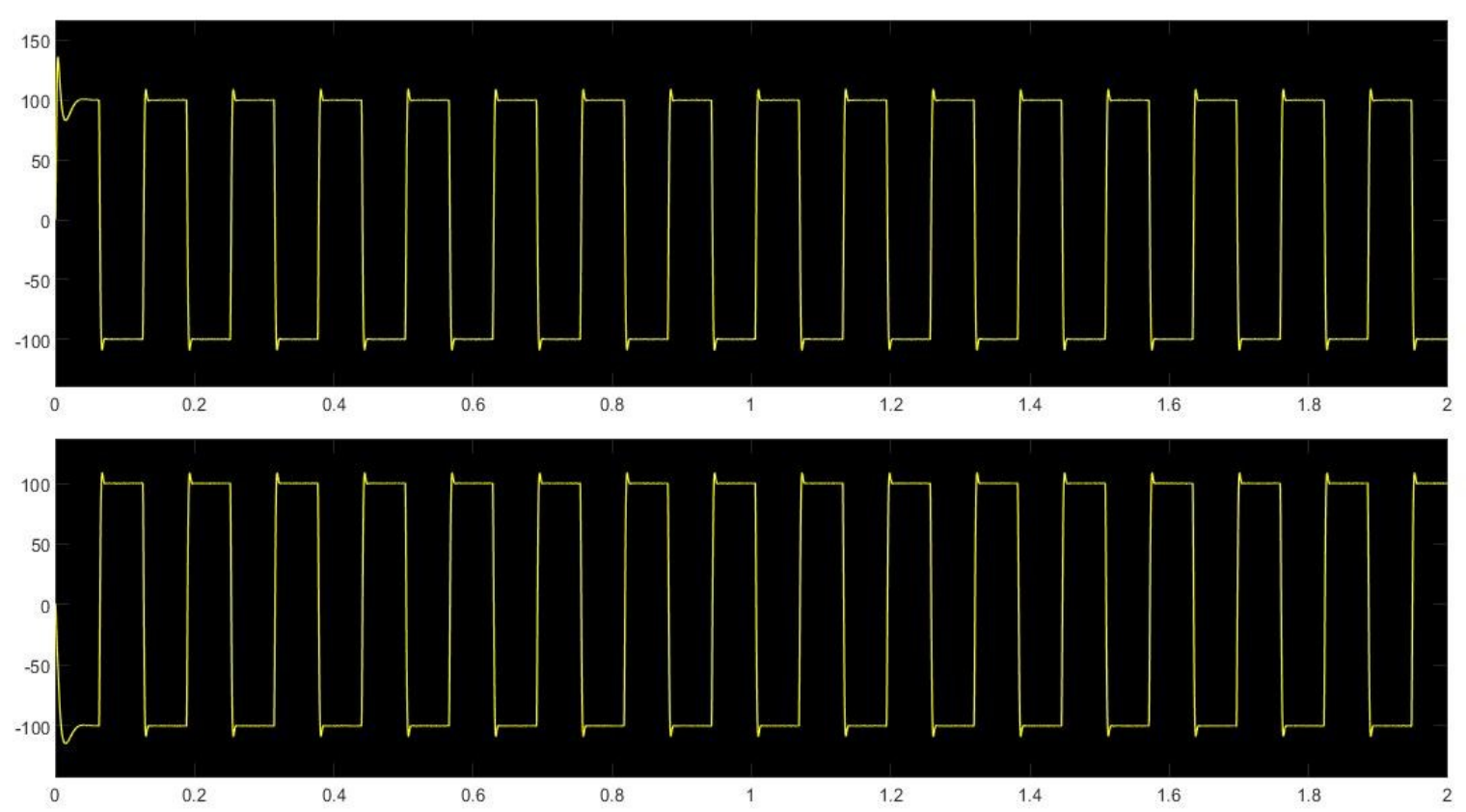

Figure 4-7 The simulation of demodulated data for QPSK Costas loop phase acquisition

Compared with the two BPSK data in Figure 4-5, it is obvious that the QPSK data can be demodulated accurately in the I and Q arm despite of the slight ripples in the beginning of the acquisition process.

\section{- Frequency acquisition}

When the initial frequency of VCO differs from the input frequency, it takes time for the signal to steer the VCO to achieve synchronization. In Figure 4-3, the initial frequency of VCO is set to be $99,500 \mathrm{rad} / \mathrm{s}$ and the input frequency is $100,000 \mathrm{rad} / \mathrm{s}$. Hence the initial frequency difference is $500 \mathrm{rad} / \mathrm{s}$.

One parameter needs to be focused on is that, different from BPSK Costas loop, there is an intersect multiplication between the two arms. For example, the signal after the 
hard-limiting function in I arm will multiply the signal after the LPF in the Q arm. In this process, the multiplication of two functions will double the frequency of the output signal along with higher harmonics. Then the difference function of the two arms will again double the frequency by cancelling out the second harmonics. In other words, the frequency difference presented at the loop filter will be four times the initial frequency difference.

The change of the shape of the waveform can also be tested in a simple simulation as shown in Figure 4-8.

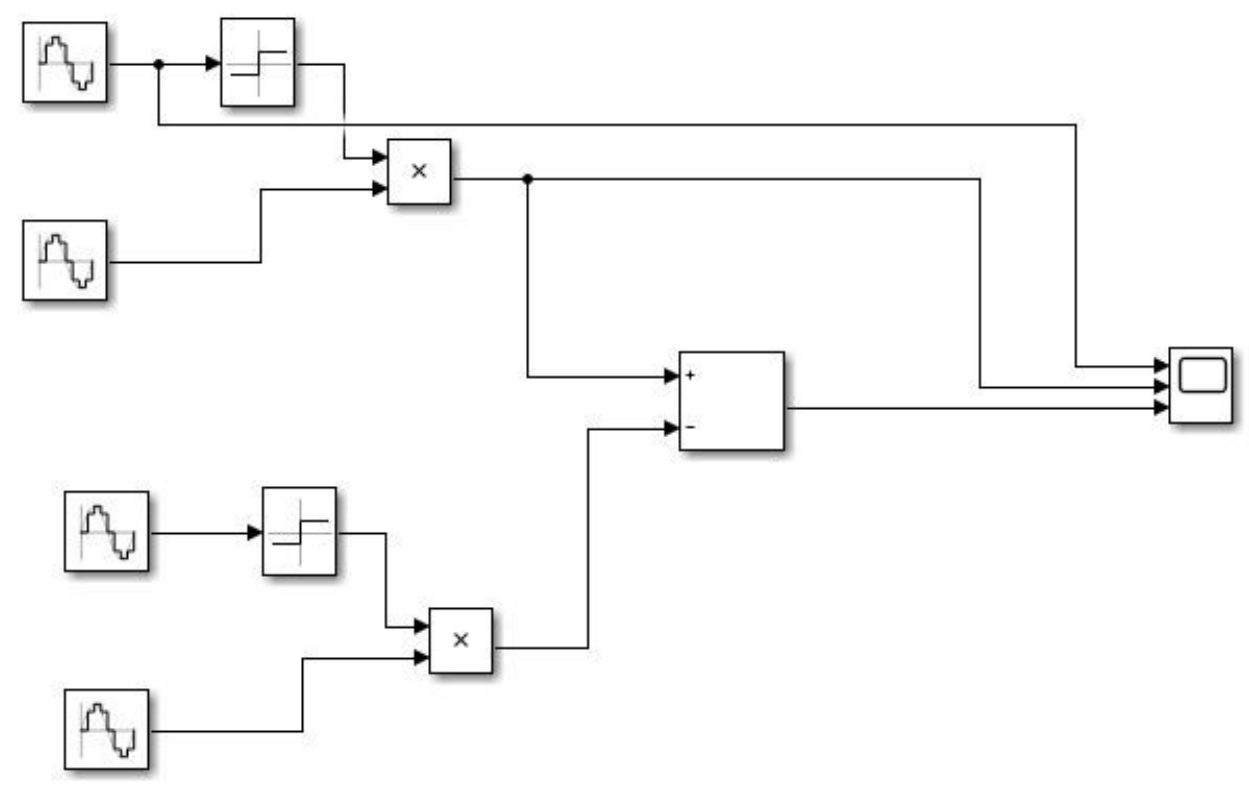

Figure 4-8 The test for the multiplication between sign function and sinusoidal function signals

The results are shown in Figure 4-9. 

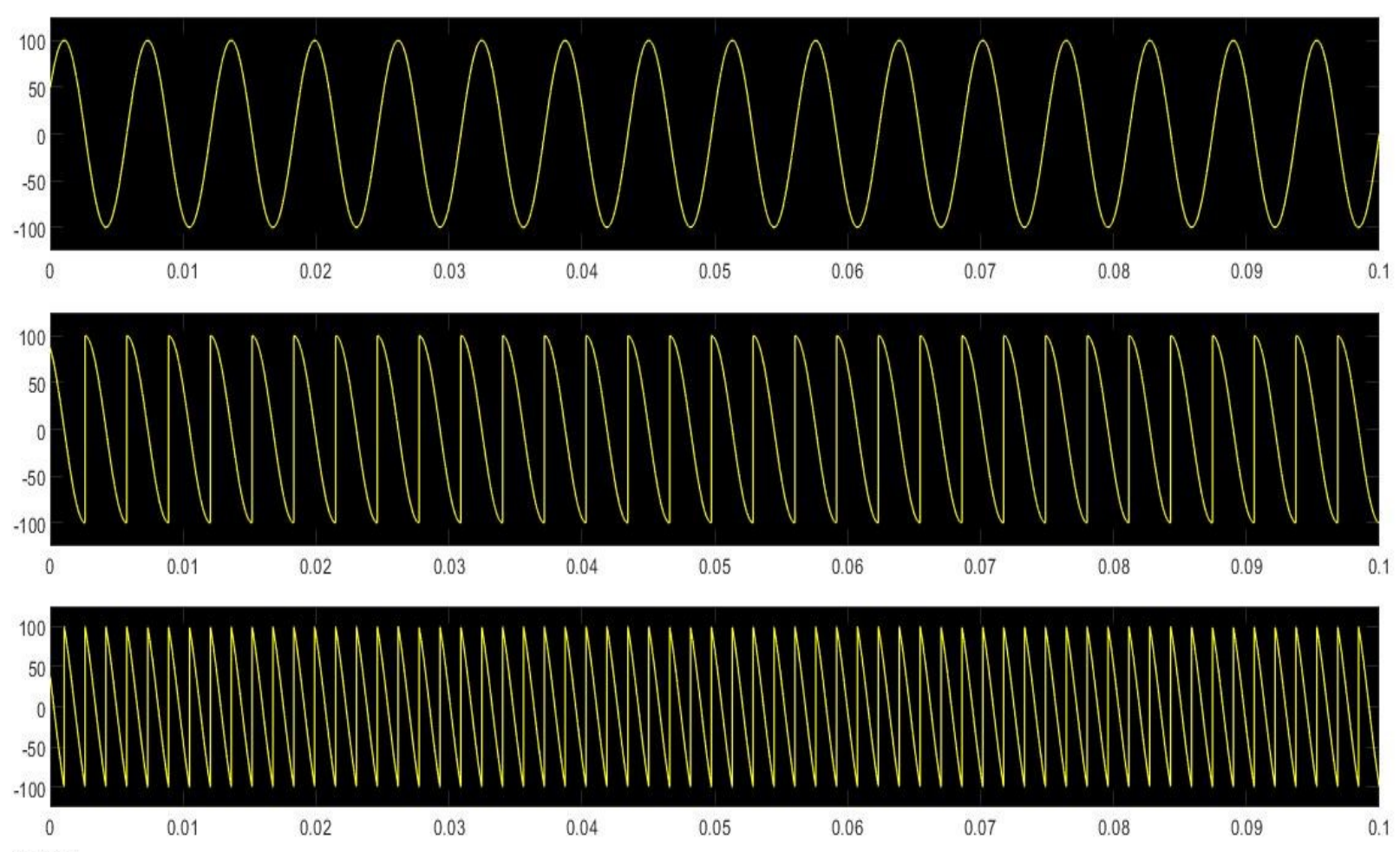

Offset $=0$

Figure 4-9 The Simulation results for testing the frequency change of different process in QPSK Costas loop

From Figure 4-9, we can see that the frequency difference will be four times the original frequency, which illustrates that frequency bandwidth for the LPF in both I and Q arm should be at least four times the original frequency difference.

Using the same values for the parameters, the simulation of demodulated data is shown in Figure 4-10, and the waveform before the loop filter and VCO is shown in Figure 4-11. 

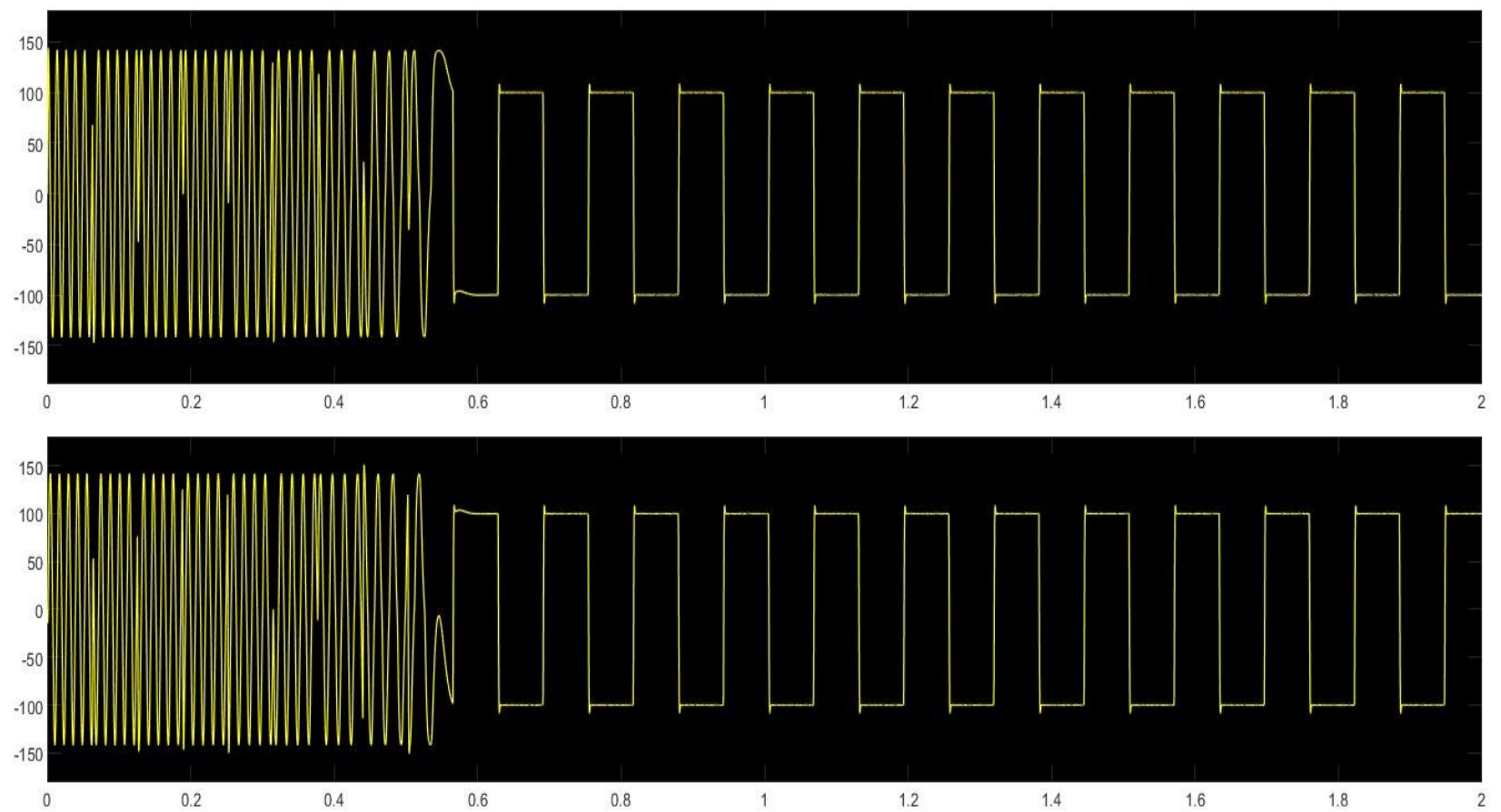

Figure 4-10 The demodulated data for frequency acquisition for a QPSK Costas loop
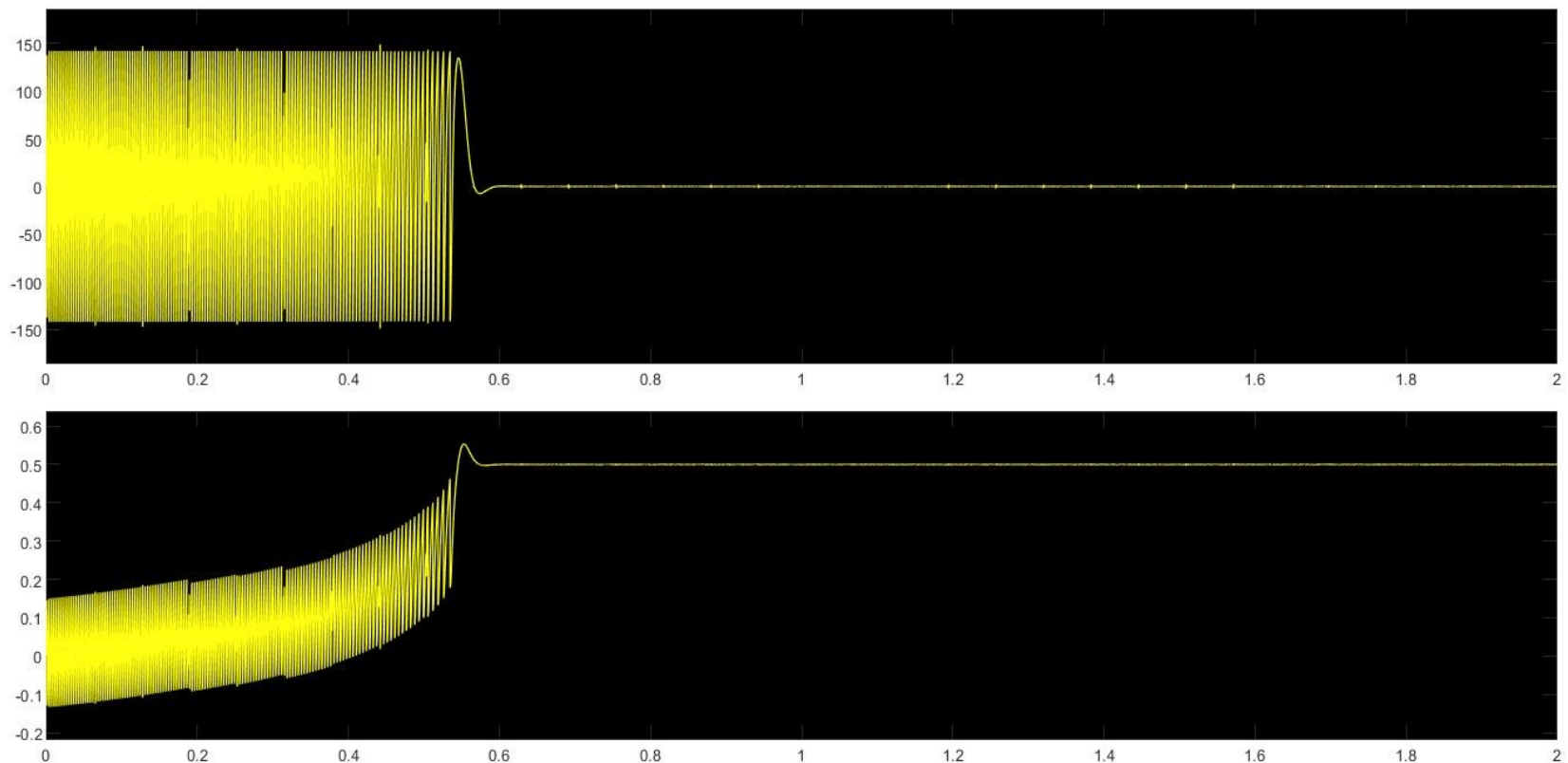

Figure 4-11 The waveform before the loop filter and the VCO 


\subsection{Fast Acquisition and the Impossibility of using a quadri-}

\section{correlator in QPSK}

- Fast acquisition and the parameters

As for BPSK, one of the ways of achieving fast synchronization is to change the parameters of the loop filter. Recall the analysis of equation 3-35, and if the acquisition time needs to be shortened, the value of the parameter $\tau_{1}$ needs to decrease. Compared with the previous function of the loop filter $4-8$, we can decrease the value of $\tau_{1}$, and see how the loop responds. The new function of loop filter is shown in 4-9 with $\tau_{1}=8 \mathrm{~s}$.

$$
F(s)=\frac{0.01 s+1}{8 s}
$$

The new demodulated QPSK signals are shown in Figure 4-12:
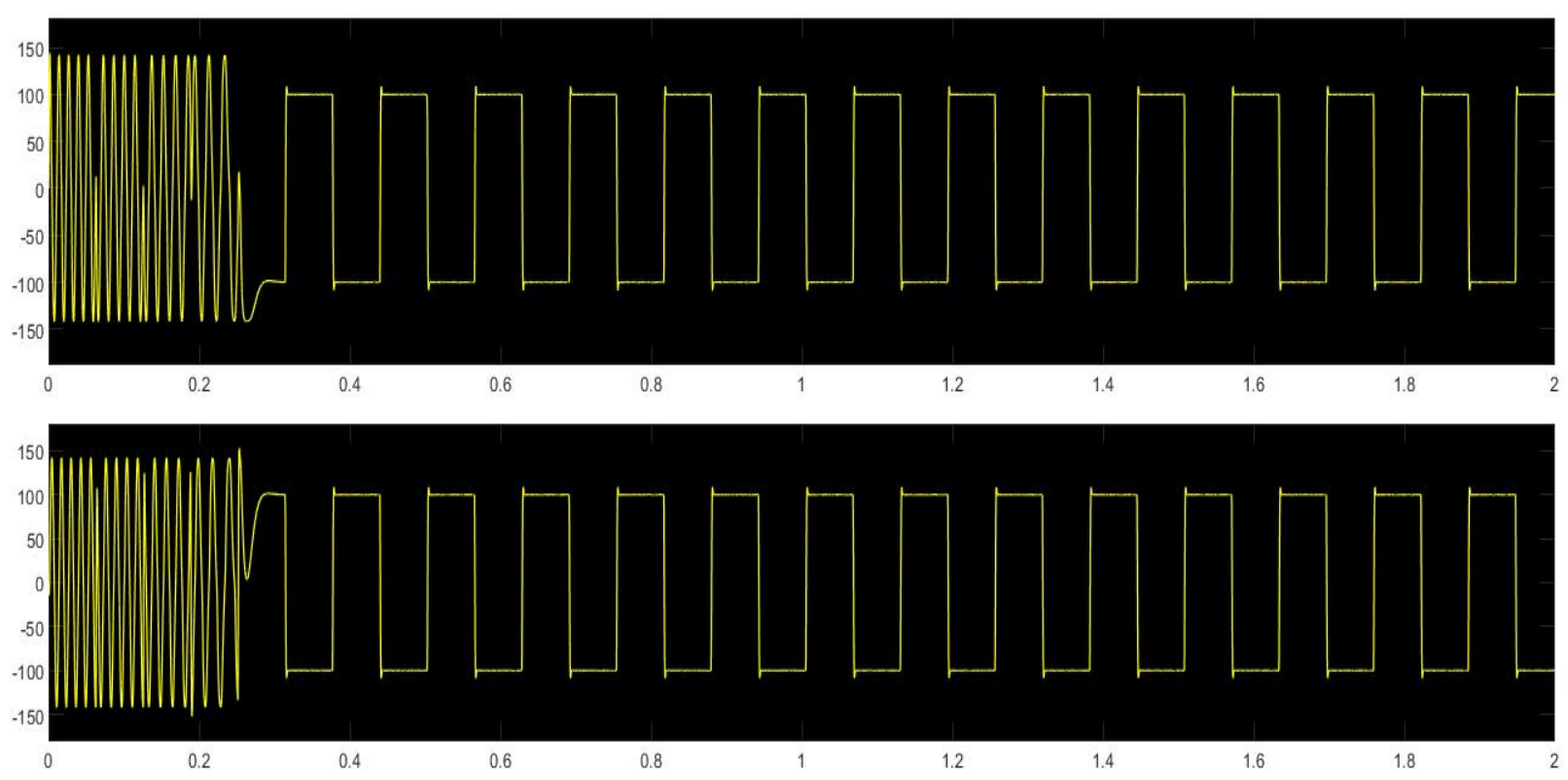

Figure 4-12 The Demodulated QPSK signals with smaller value of $\tau_{1}$ 
Compared with the simulation results in Figure 4-10, the new simulation in Figure 4-12 gives noticeable shorter time of achieving synchronization.

\section{- The impossibility of using quadri-correlator in QPSK}

In Chapter 3.4, the thesis presented a new contribution of achieving fast synchronization by adding a quadri-correlator and created an optimized way of achieving acquisition with high quality in both fast speed and less distortion. Unfortunately, the quadri-correlator is not suitable for the QPSK Costas loop.

In the BPSK Costas loop, although the derivate function in the I arm changes the function, it still preserves the style of the sinusoidal function (e.g. $\left.\frac{d \sin \omega t}{d t}=\omega \cos \omega t\right)$.

In the QPSK Costas loop, there is a hard-limiting function in the two arms, which changes the real sinusoidal function; consequently, the derivate of the results cannot be a sinusoidal function. We can set up a simple simulation to test the signals.

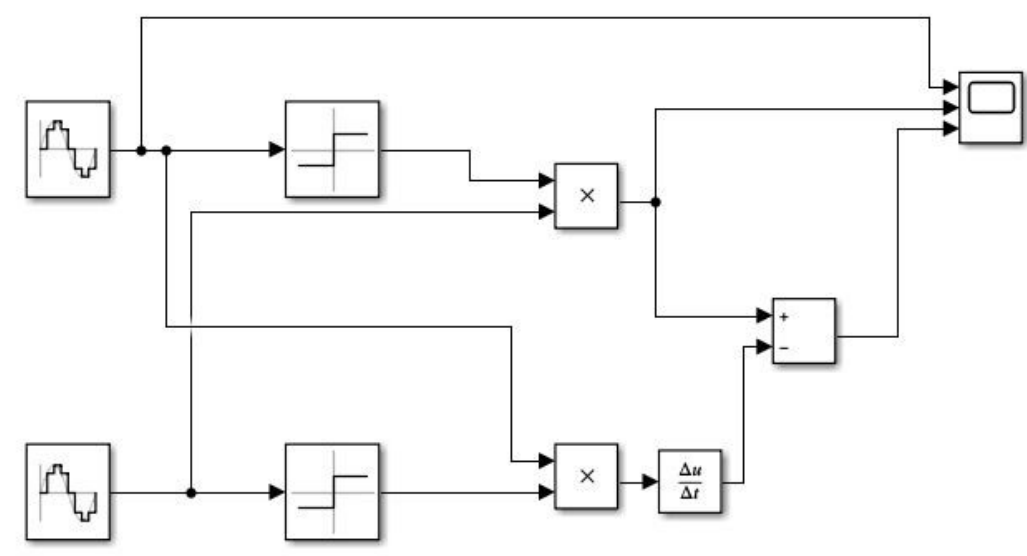

Figure 4-13 Test for the quadri-correlator added in QPSK 

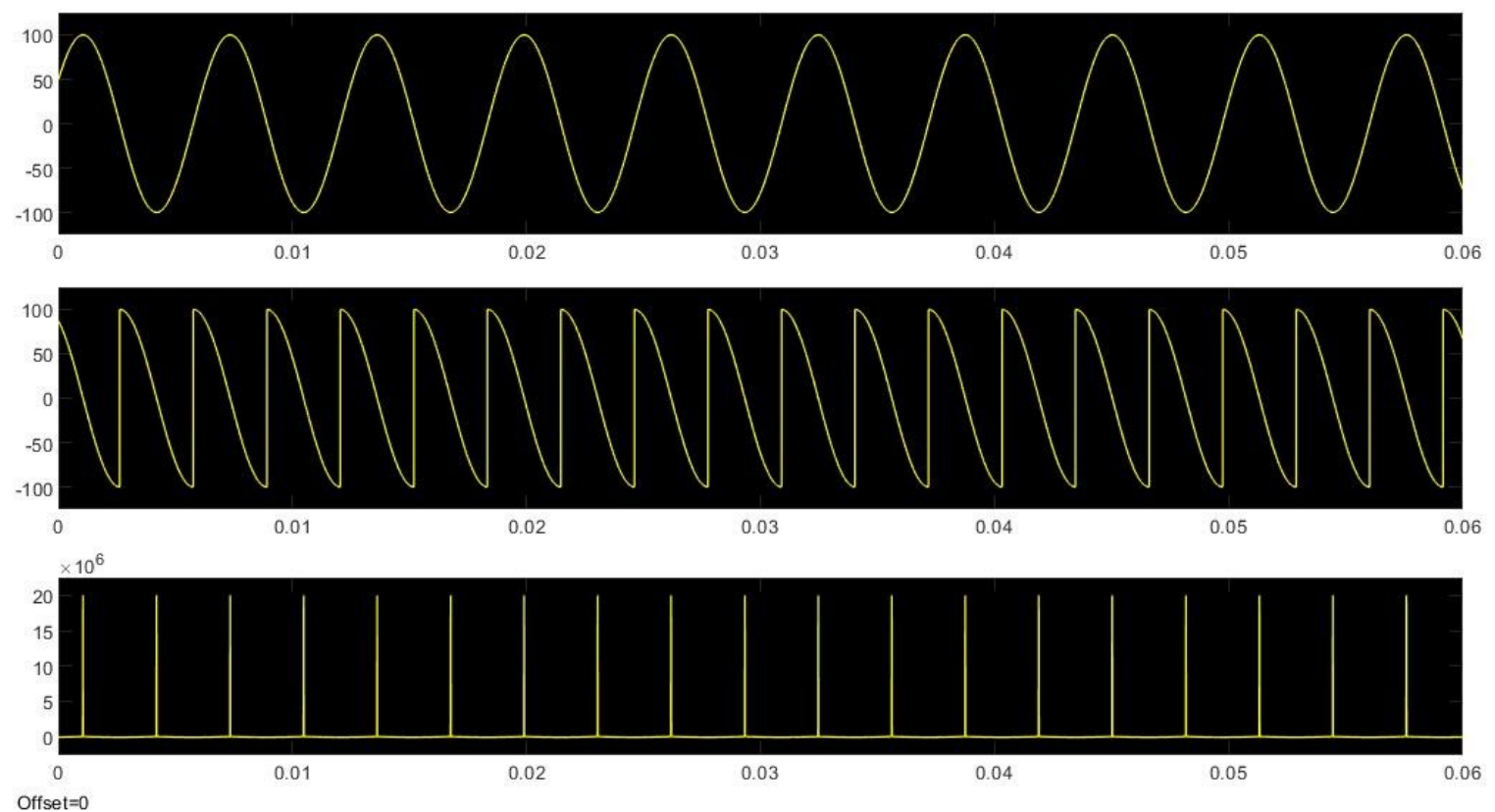

Figure 4-14 The simulation results for testing the QPSK signals with quadri-correlator

As shown in Figure 4-14, the third simulation result reflects the combination of two signals after adding the derivate function, and it is just an impulse function with an extreme high amplitude, which cannot be used to modulate the VCO to achieve synchronization. In a summary, adding a quadri-correlator in the QPSK Costas loop to attain quick acquisition cannot be achieved.

\subsection{Summary}

Mastering the characters of a QPSK Costas loop, we understand how the waveform will change in the different processes of the loop. This chapter finishes with the acquisition and demodulation using a QPSK Costas loop although the quadri-correlator cannot aid to increase synchronization. In the next chapter, the thesis will link the Doppler frequency 
change function of a LEO orbit in Chapter 2 and the designed circuits in Chapter 3 and 4 to see how the Costas loop can track and demodulate data from a LEO of Telesat's program. 


\section{Chapter 5}

\section{Doppler Frequency Tracking for a LEO}

In Chapter 2, the analysis illustrated the behaviour of the Doppler frequency shift when the terminal station receives the signal from a LEO. The normalized Doppler frequency shift function can be approximately as equation 2.17 and is shown in Figure 28. Then at the end of the Chapter 2, it was concluded that the initial normalized frequency shift will be $\frac{\Delta f}{f}=1.15 \times 10^{-5}$, and $\Delta f$ will be $3.45 \times 10^{5} \mathrm{~Hz}=2,167,698 \mathrm{rad} / \mathrm{s}$, at the beginning of the information transition.

\subsection{Doppler frequency shift tracking for BPSK signals}

In the Simulink, the down converted carrier frequency from the LEO satellite is $40,000,000 \mathrm{rad} / \mathrm{s}$. Since we know the estimation of the frequency shift caused by Doppler shift, to decrease the acquisition time, an initial frequency offset of the VCO can be used. In simulation, the initial VCO frequency has been chosen to be $42,160,000 \mathrm{rad} / \mathrm{s}$. As a result, frequency difference is reduced to be $7,698 \mathrm{rad} / \mathrm{s}$. 
The new Costas loop design, using a combination of the traditional BPSK Costas loop and the BPSK Costas loop with a quadri-correlator is used. This loop is shown in Figure 3-27

\section{Parameters for the loop:}

- The bandwidth of low pass filter in both two arms: $20000 \mathrm{rad} / \mathrm{s}$.

- The amplitude of the carrier frequency: $100 \mathrm{~V} / \mathrm{rad}$

- The sensitivity of the VCO: $10000 \mathrm{rad} / \mathrm{s} / \mathrm{V}$.

- The loop filter for the traditional Costas loop:

$$
F(s)=\frac{0.01 s+1}{2500 s}
$$

- The loop for the Costas loop with aided a quadri-correlator:

$$
F(s)=\frac{0.01 s+1}{2500 \times 7698 s}
$$

The BPSK Costas loop incorporating a quadri-correlator tracking of the incoming signals in Simulink is shown in Figure 5-1, and the results of the demodulation are shown in Figure 5-2. The function block in Figure 5-1 denotes the expected incoming carrier frequency affected by Doppler frequency shift. 


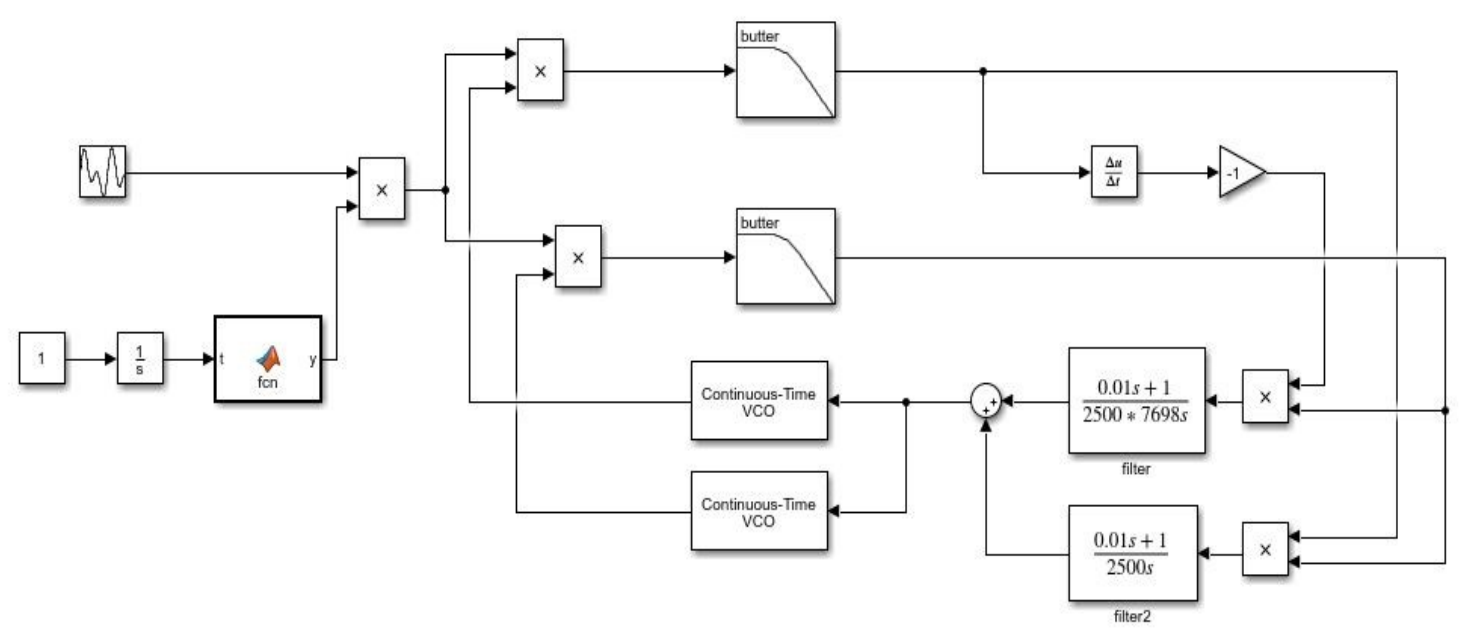

Figure 5-1 Doppler sift tracking with BPSK Costas loop in Simulink
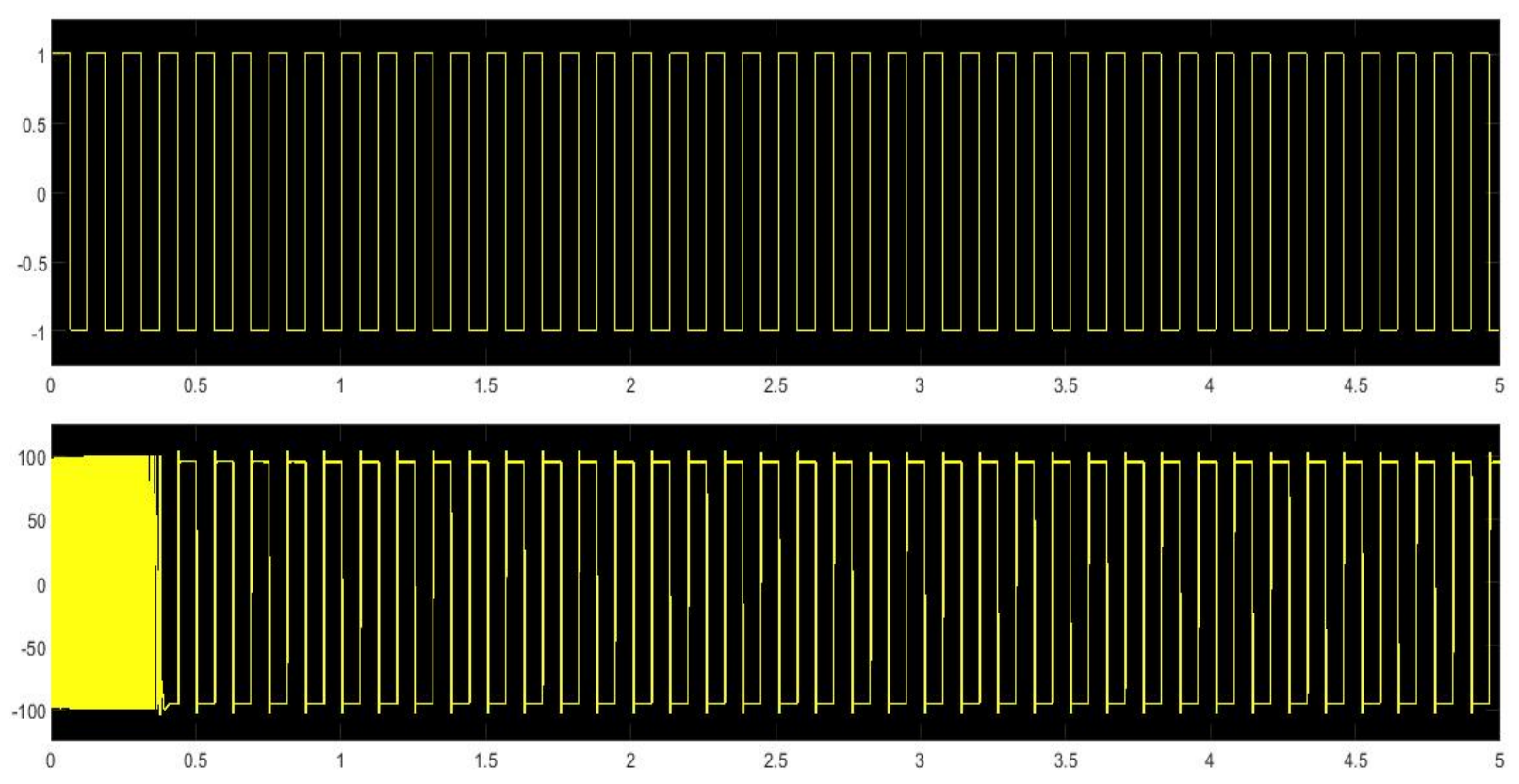

Figure 5-2 The simulation for demodulation of BPSK data when the terminal station receives the data from a LEO 


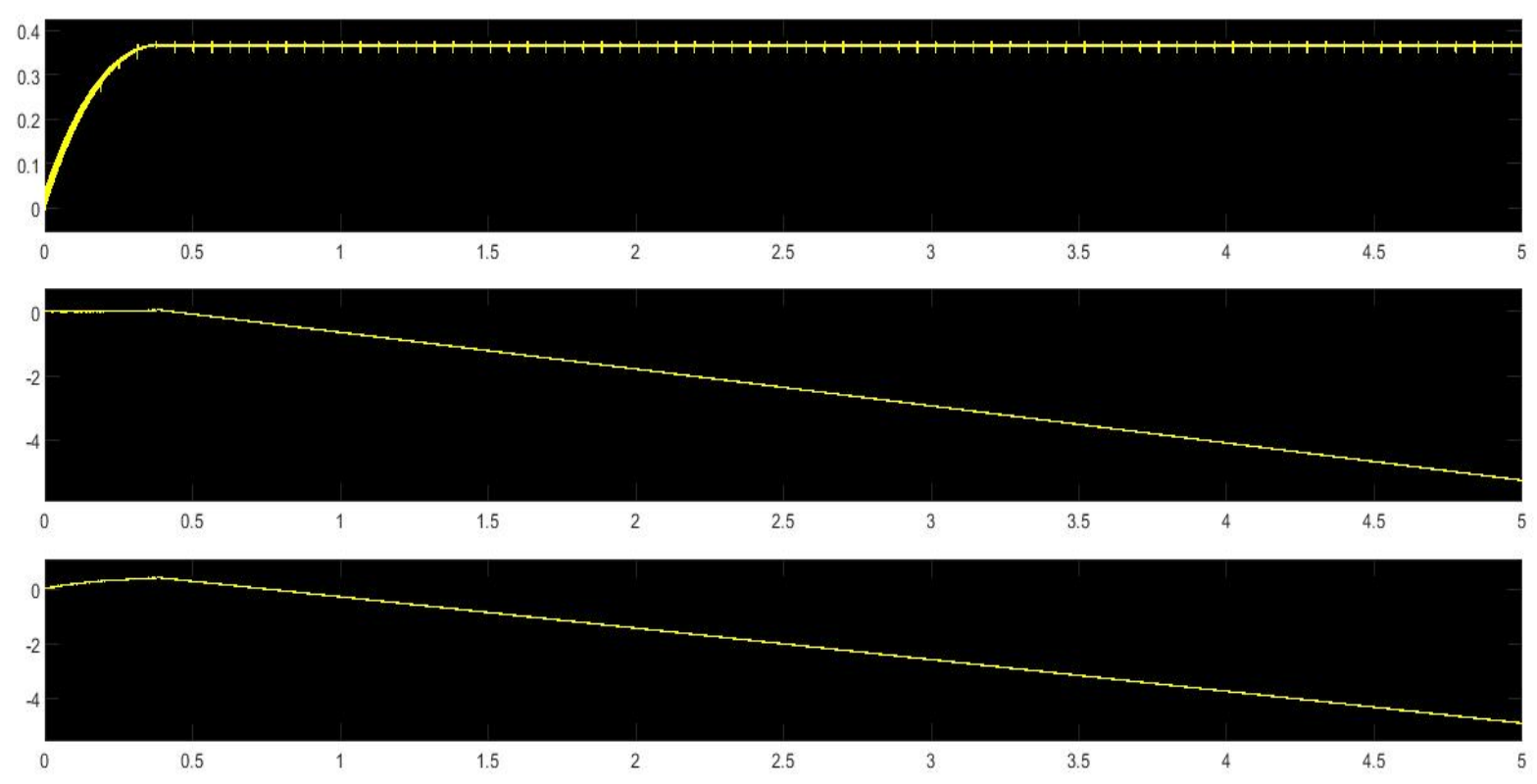

Figure 5-3 The simulation of the signal after the three filters. (1) the signal after the loop filter of the quadri-correlator Costas loop (2) the signal after the loop filter of the traditional Costas loop (3) the signal of the combination of two loop filters (the numbers are from vertical order)

From the figure 5-2 we can see that at $0.4 \mathrm{~s}$, the loop achieves synchronization and BPSK signals can be demodulated.

The demodulation time can continue to decrease by choosing a smaller value of $\tau_{1}$ if the synchronization demands a higher speed of acquisition.

\subsection{Doppler frequency shift tracking for QPSK signals}

Apart from a better estimation of the initial arrival frequency to increase the synchronization speed, and different from the BPSK Costas loop being able to use a quadricorrelator for speeding up the acquisition, a third way of accelerating synchronization can be achieved by choosing a smaller value of $\tau_{1}$. Here we use this for the QPSK Costas loop. 
As in the above simulations, after an initial Doppler frequency offset estimation, the original frequency of the VCO can chosen to be $42,160,000 \mathrm{rad} / \mathrm{s}$.

\section{Parameters for the loop:}

- The bandwidth of low pass filter in both two arms: $40000 \mathrm{rad} / \mathrm{s}$.

- The amplitude of the carrier frequency: $100 \mathrm{~V} / \mathrm{rad}$

- The sensitivity of the VCO: $10000 \mathrm{rad} / \mathrm{s} / \mathrm{V}$.

- The loop filter for the traditional Costas loop: $F(s)=\frac{0.01 s+1}{10 s}$

(To accelerate the speed of achieving synchronization, the parameter $\tau_{1}$ change from 100 s to 10 s)

Here the QPSK Costas loop tracking the incoming signals in Simulink is shown in Figure 5-4, and the results of the demodulation are shown in Figure 5-5. Figure 5-6 denotes the expected incoming carrier frequency affected by Doppler frequency shift.

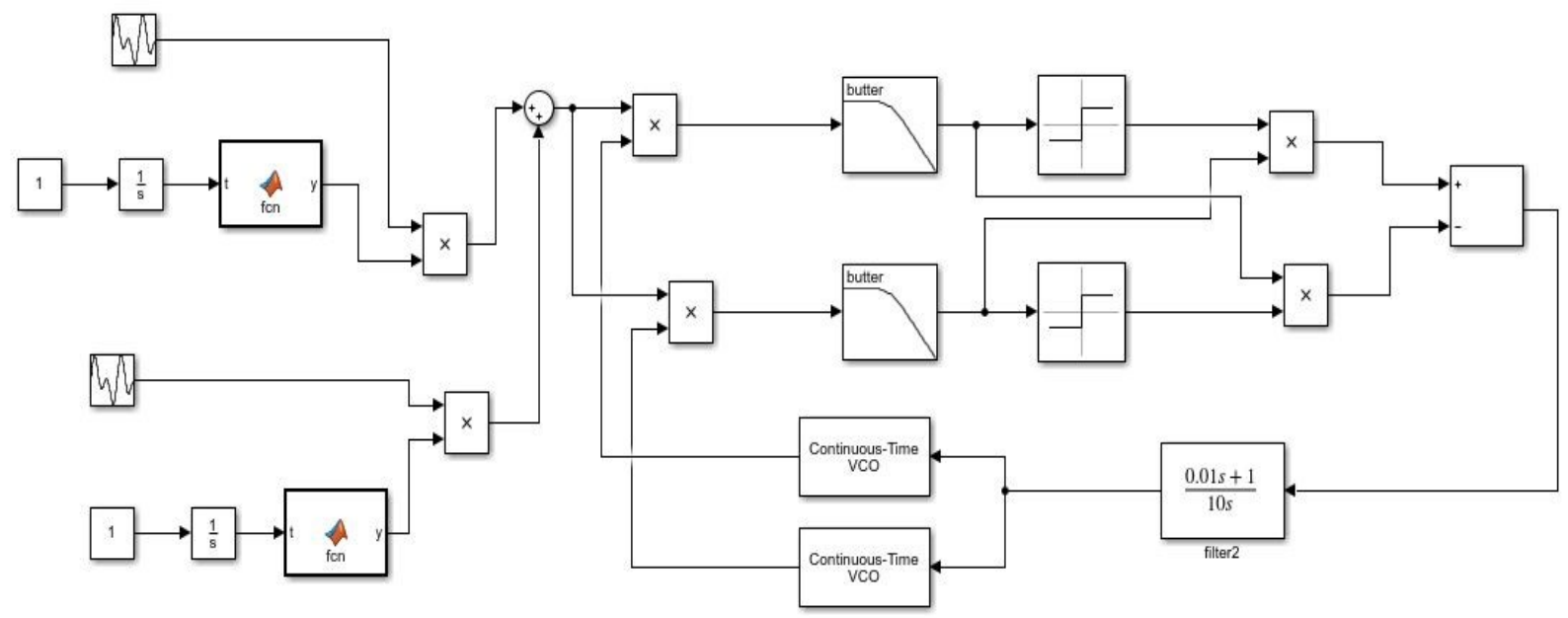

Figure 5-4 Doppler shift tracking with QPSK Costas loop in Simulink 

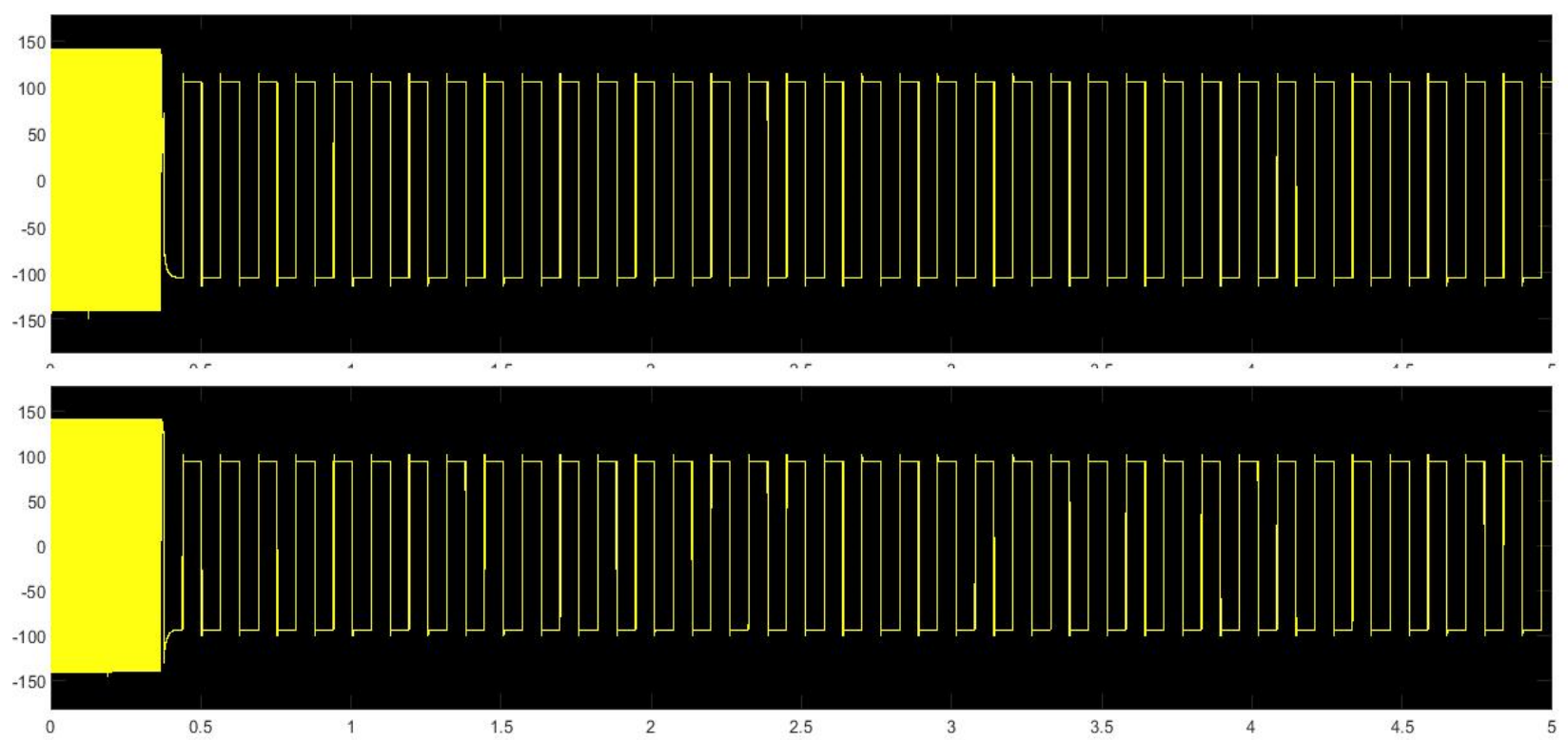

Figure 5-5 The simulation for demodulation of QPSK data when the terminal station receives the data from Telesat LEO (1) The demodulated signals of $m(t)$ (2) The demodulated signals of $n(t)$

Figure 5-5 illustrates that at $0.4 \mathrm{~s}$, the QPSK Costas loop achieves synchronization and demodulates the signals.

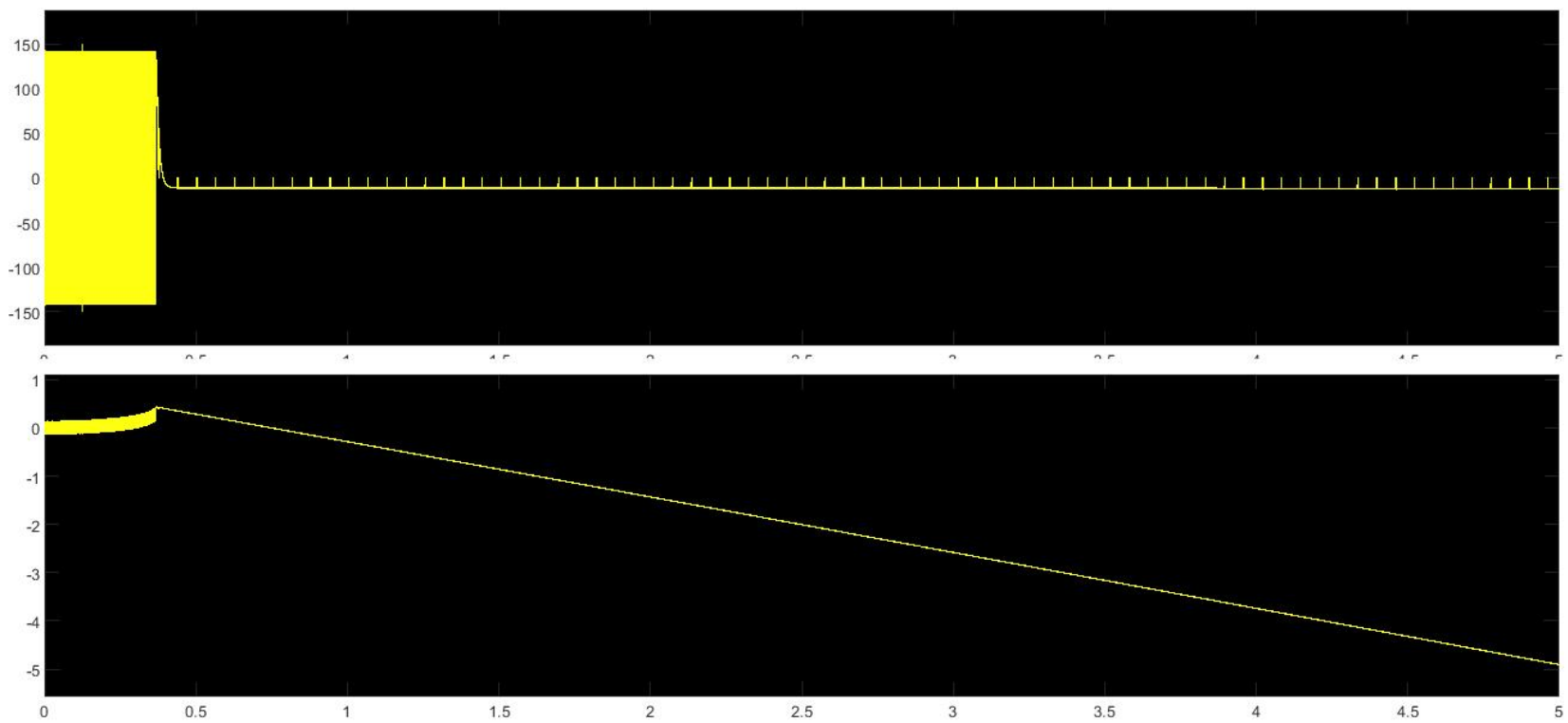

Figure 5-6 The simulation of the signals before and after the loop filter in QPSK Costas loop. (1) the signals after before the loop filter (2) the signals after the loop 


\subsection{Summary}

Combing the theory and the function of Chapter 2, 3 and 4, this Chapter shows the simulation of the designed BPSK Costas loop and QPSK Costas loop when the signals are received from a LEO satellite. Figure 5-3 and 5-5 clearly present the demodulated data, although as always, the residual data ambiguity exists in the demodulated signal. 


\section{Chapter 6}

\section{Conclusion}

Using known parameters provided by Telesat LEO, this thesis derives the estimated Doppler frequency shift time profile from a LEO satellite to a terrestrial terminal station. During the process of receiving and demodulating data, the thesis contributes two new designs for a BPSK Costas loop to track the incoming frequency, one is the traditional Costas loop with a quadri-correlator in one of the two arms, and the other one is the combination of the traditional Costas loop and the Costas loop with a quadri-correlator. Both of the new designs can increase the speed of synchronization, albeit with some distortion at the beginning of the synchronization process for the BPSK Costas loop with a quadri-correlator. The second type of the new design optimizes synchronization to a great extent including both speed and waveform distortion. When the original frequency difference is $1000 \mathrm{rad} / \mathrm{s}$, the combination of traditional Costas loop and the Costas loop with a quadri-correlator can achieve synchronization at around $0.1 \mathrm{~s}$ compared with the Costas loop without any auxiliary circuit being around $0.4 \mathrm{~s}$, This represents an improvement of $400 \%$. For Costas loop without any auxiliary circuits, it was shown that the acquisition time can be continually reduced by using smaller values of $\tau_{1}$.

As for the QPSK Costas loop, the thesis demonstrates the impracticality of the construction of the QPSK Costas loop with the function of a quadri-correlator. This shows the impossibility of adding a quadri-correlator to a QPSK Costas loop to help synchronization. However, the acquisition time can still be reduced by choosing a smaller 
value of $\tau_{1}$. In the real case of tracking signals for a LEO satellite, a synchronization time of $0.4 \mathrm{~s}$ can be achieved by decreasing the value of $\tau_{1}$.

\subsection{Future work}

Although the acquisition time can be reduced by selecting a smaller value of $\tau_{1}$, according to $\frac{K}{\omega_{n}^{2}}=\tau_{1}$, the lower value of $\tau_{1}$ will indicate the higher value of natural frequency, $\omega_{n}$. However, the value of parameter $\omega_{n}$ is often expected to be relatively low in the second-order loop, which results in a lower angular frequency value when the unity gain $G$ is equal to $0 \mathrm{~dB}$. Then in the function of the phase detector $\frac{K_{1}}{1+\tau s}$, and $\operatorname{VCO} \frac{K_{3}}{1+\tau^{\prime} s}$, parameters $\tau$ and $\tau^{\prime}$ can be both ignored, and we can get a better approximation of the second-order loop filter function [17]. An optimized circuit design methodology for acquisition time could be undertaken.

In the LEO satellite communication and $5 \mathrm{G}$ requirement, the speed is one of the dominant factors to consider, so there is high demand for achieving synchronization in a short time. As a result, the future work will be allocated in creating new auxiliary circuits for QPSK to achieve faster synchronization different from adding a quadri-correlator and BPSK to achieve further optimization. Also, dealing with noise performance and nonlinear distortion effects need to be studied in the acquisition process. 


\section{Reference List}

[1] S. Mosunmola B, O. Agboola, A. Felix , and A. Mohammed, "The Mathematical Model of Doppler Frequency Shift in Leo At Ku, K and Ka Frequency Bands," International Journal of Trend in Research and Development (IJTRD) ISSN: 23949333, vol. 4, no. 5, Oct. 2017.

[2] I.F. Akyildiz, H. Uzunalioğlu, and M.D. Bender, "Handover management in low earth orbit (LEO) satellite networks." Mobile Networks and Applications 4, pp. 301310,1999

[3] O. Kodheli, A. Guidotti, and A. Vanelli-Coralli, "Integration of Satellites in 5G through LEO Constellations," GLOBECOM 2017 - 2017 IEEE Global Communications Conference, pp. 1-6, 2017.

[4] C. Fossa, R. Raines, G. Gunsch, and M. Temple, "An overview of the IRIDIUM (R) low Earth orbit (LEO) satellite system," Proceedings of the IEEE 1998 National Aerospace and Electronics Conference. NAECON 1998. Celebrating 50 Years (Cat. No.98CH36185), pp. 152-159, 1998.

[5] C. Chen and E. Ekici, "A Routing Protocol for Hierarchical LEO/MEO Satellite IP Networks," Wireless Networks, vol. 11, no. 4, pp. 507-521, 2005.

[6] P. Narvaez, A. Clerget, and W. Dabbous, "Internet routing over LEO satellite constellations," In Third ACM/IEEE International Workshop on Satellite-Based Information Services (WOSBIS'98). 1998.

[7] S. Amiri and M. Mehdipour, "Accurate Doppler Frequency Shift Estimation for any Satellite Orbit," 2007 3rd International Conference on Recent Advances in Space Technologies, pp. 602-607, 2007.

[8] G.-C. Hsieh and J. Hung, "Phase-locked loop techniques. A survey," IEEE Transactions on Industrial Electronics, vol. 43, no. 6, pp. 609-615, 1996.

[9] X. Guo, W. Wu, and Z. Chen, "Multiple-Complex Coefficient-Filter-Based PhaseLocked Loop and Synchronization Technique for Three-Phase Grid-Interfaced Converters in Distributed Utility Networks," IEEE Transactions on Industrial Electronics, vol. 58, no. 4, pp. 1194-1204, 2011. 
[10] "Telesat Global LEO Constellation," https://static1.squarespace.com/static/5274112ae4b02d3f058d4348/t/5e0c5916a5b0832cb78c2db4/1577867573707/201 9-3-2f-3.pdf, Nov-2019. .

[11] M. Neri, "Telesat LEO Fiber quality connectivity everywhere," https://www.itu.int/dms_pub/itu-r/oth/0a/OE/R0A0E0000B90001PDFE.pdf, Jun2018. .

[12] I. Ali, N. Al-Dhahir, and J. Hershey, "Doppler characterization for LEO satellites," IEEE Transactions on Communications, vol. 46, no. 3, pp. 309-313, 1998.

[13] I. Ali, N. Al-Dhahir, and J. Hershey, "Predicting the visibility of LEO satellites," IEEE Transactions on Aerospace and Electronic Systems, vol. 35, no. 4, pp. 11831190, Oct. 1999.

[14] D. B. Tablot, "Nonsweep Acquisition Methods,” pp. 85-103, 2012.

[15] R. E. Best, N. V. Kuznetsov, G. A. Leonov, M. V. Yuldashev, and R. V. Yuldashev, "Simulation of Analog Costas Loop Circuits," International Journal of Automation and Computing, 11(6), pp. 571-579, 2014.

[16] R. Best, N. Kuznetsov, G. Leonov, M. Yuldashev, and R. Yuldashev, "Tutorial on dynamic analysis of the Costas loop," Annual Reviews in Control, vol. 42, pp. $27-$ 49, 2016.

[17] A. Blanchard, Phase-locked loops: application to coherent receiver design. John Wiley \& Sons, 1976.

[18] J. G. Proakis and M. Salehi, Digital Communications Fifth Edition. New York: McGraw-Hill, 2008. 